Published in final edited form as:

Compr Physiol. 2012 April ; 2(2): 1303-1353. doi:10.1002/cphy.c110041.

\title{
Pathophysiology of Acute Kidney Injury
}

\author{
David P. Basile ${ }^{1}$, Melissa D. Anderson ${ }^{2}$, and Timothy A. Sutton ${ }^{2}$ \\ ${ }^{1}$ Department of Cellular \& Integrative Physiology, Indiana University School of Medicine, \\ Indianapolis, IN \\ ${ }^{2}$ Department of Medicine, Division of Nephrology, Indiana University School of Medicine, \\ Indianapolis, IN
}

\begin{abstract}
Acute kidney injury (AKI) is the leading cause of nephrology consultation and is associated with high mortality rates. The primary causes of AKI include ischemia, hypoxia or nephrotoxicity. An underlying feature is a rapid decline in GFR usually associated with decreases in renal blood flow. Inflammation represents an important additional component of AKI leading to the extension phase of injury, which may be associated with insensitivity to vasodilator therapy. It is suggested that targeting the extension phase represents an area potential of treatment with the greatest possible impact. The underlying basis of renal injury appears to be impaired energetics of the highly metabolically active nephron segments (i.e., proximal tubules and thick ascending limb) in the renal outer medulla, which can trigger conversion from transient hypoxia to intrinsic renal failure. Injury to kidney cells can be lethal or sublethal. Sublethal injury represents an important component in AKI, as it may profoundly influence GFR and renal blood flow. The nature of the recovery response is mediated by the degree to which sublethal cells can restore normal function and promote regeneration. The successful recovery from AKI depends on the degree to which these repair processes ensue and these may be compromised in elderly or CKD patients. Recent data suggest that AKI represents a potential link to CKD in surviving patients. Finally, earlier diagnosis of AKI represents an important area in treating patients with AKI that has spawned increased awareness of the potential that biomarkers of AKI may play in the future.
\end{abstract}

\section{Introduction}

In the century that followed Richard Bright's description of kidney disease in 1827 (72) many case studies of acute Bright's disease associated with a variety of etiologies including infections, toxins, and transfusion reactions were published. However, it was the landmark report by Bywaters and Beal in 1941 (84) linking crush injury to the acute impairment of renal function that stands out as the starting point for modern medicine's discussion of acute kidney injury (AKI). This ongoing discussion in the clinic and laboratory has made significant strides in our understanding of AKI, formerly known as acute renal failure (ARF). Nonetheless, AKI remains a significant and serious problem accounting for the most common reason for nephrology consultation in the hospitalized patient. In fact, the progress of modern medicine and the aging population has primarily rendered AKI a nosocomial disease in the developed world. It is the intent of this chapter to provide our current understanding of cell, organ, and systemic alterations involved in the pathophysiology of AKI that will hopefully serve as a framework on which to build further understanding of this vexing syndrome and ultimately provide meaningful therapeutic interventions in the clinic. 


\section{AKI: History, Etiology, Terminology and Pathology}

\section{I.1. Etiology}

One of the most important functions of the kidney is the filtration and excretion of nitrogenous waste products from the blood. The measurements of elevated blood urea nitrogen (BUN) and creatinine serve as indicators of decreased renal function indicative of the decreased clearance of these waste products. AKI is currently defined as a rapid decline in the glomerular filtration rate (GFR) resulting in retention of nitrogenous wastes, primarily creatinine and blood urea nitrogen (542). Consequently, the diagnosis currently is dependent on the serial measurement over time of these substances in the blood of patients. The rapidity of this decline may occur over a time course of hours to months, but typically occurs over the course of hours to days. The ability of these measurements to serve as a surrogate marker of GFR is relatively imprecise $(138,363)$ and improved methods for evaluating GFR and a direct assessment of renal injury are sorely needed in the practice of medicine (96).

Clinically, AKI can be conveniently grouped into three primary etiologies: prerenal, renal, and postrenal (542). All three etiologies will be discussed briefly here; however, renal etiologies, especially those from ischemic and nephrotoxic injury, will receive the bulk of the discussion in this chapter given the frequency with which they occur and the fact that they are the etiologies associated with frank renal tissue injury.

Maintaining a normal GFR is dependent on adequate renal perfusion. Prerenal azotemia is characterized by a decrease in GFR due to a decrease in renal perfusion pressure without damage to the renal parenchyma (22). The kidneys receive up to $25 \%$ of the cardiac output and thus any failure of the general circulation or isolated failure of the intrarenal circulation can have a profound impact on renal perfusion. Causes of prerenal azotemia include: hypovolemia resulting from conditions such as hemorrhage, vomiting, diarrhea "third spacing," poor oral intake, burns, excessive sweating, renal losses (e.g. diuresis); impaired cardiac output resulting from congestive heart failure or decreased cardiac output states (e.g. pericardial tamponade, severe pulmonary hypertension); decreased vascular resistance (peripheral vasodilation) resulting from conditions such as sepsis, vasodilator medications, autonomic neuropathy, or anaphylaxis; and renal vasoconstriction from vasoconstrictive medications or conditions such as hypercalcemia.

The normal response of the kidney to prerenal conditions is to concentrate the urine maximally and avidly reabsorb sodium in an effort to maintain/increase intravascular volume and normalize renal perfusion (372). In general, therapy aimed at rapidly restoring renal perfusion will promptly improve renal function. However, it is important to appreciate that prolonged or profound prerenal azotemia can result in ischemic damage to the kidney and thus there is spectrum from prerenal azotemia to ischemic AKI.

Postrenal causes of AKI are characterized by acute obstruction to urinary flow. Urinary tract obstruction increases intratubular pressure and thus decreases GFR. In addition, acute urinary tract obstruction can lead to impaired renal blood flow and inflammatory processes that also contribute to diminished GFR $(200,222,234,500,581)$. Obstruction of the urinary tract at any level may produce AKI. In general, obstruction must involve both kidneys or a solitary kidney to produce significant renal failure. However, a patient with pre-existing renal insufficiency may develop AKI with obstruction of only one kidney.

Urinary obstruction may present as anuria or intermittent urine flow (such as polyuria alternating with oliguria) but may also present as nocturia or nonoliguric AKI. Causes of postrenal AKI include benign prostatic hyperplasia and prostate cancer in men, gynecologic 
cancers especially cervical cancer in women, retroperitoneal fibrosis, ureteral stones, papillary necrosis, neurogenic bladder, and intratubular obstruction due to precipitation of various substances such as acyclovir or indinavir (95).

Renal etiologies of AKI can be a challenging form of AKI to evaluate because of the wide variety of injuries that can occur to the kidney. In general, it can be helpful to think of damage to the four major structures of the kidney when considering etiologies of intrinsic renal failure. These four structures are 1) the tubules, 2) the glomeruli, 3) the interstitium, and 4) the intrarenal blood vessels.

Tubular Damage-Acute tubular necrosis (ATN) is the term used to designate AKI resulting from damage to the tubules. The two major causes of ATN are:

1. Ischemic - resulting from severe or protracted decrease in renal perfusion.

2. Nephrotoxic - resulting from a variety of exogenous compounds (e.g. aminoglycosides, amphotericin B, cis-platinum, radiocontrast media) and endogenous compounds (e.g. hemoglobin in hemolysis, myoglobin in rhabdomyolysis) that are toxic or potentially toxic to the kidney.

Historically, classic ATN goes through an oliguric (urine output $\leq 400 \mathrm{~mL} / 24$ hours) phase of 1-2 weeks followed by a nonoliguric (urine output $>400 \mathrm{~mL} /$ day) phase of 10-14 days with eventual recovery of renal function. However, this description is not the rule as both prolonged oliguric phases and initial nonoliguric phases are common (381).

Glomerular Damage-AKI from glomerular damage occurs in severe cases of acute glomerulonephritis (GN). Acute GN can be due to a primary renal disease such as an idiopathic rapidly progressive GN or as part of a systemic disease such as systemic lupus erythematosus, bacterial endocarditis, or Wegener's granulomatosis $(381,542)$.

Interstitial Damage-AKI from interstitial damage can result from acute interstitial nephritis due to an allergic reaction to a variety medications (commonly antibiotics such as penicillins, cephalosporins, sulfonamides) or an infection (bacterial illnesses such as leptospirosis, legionella, rarely pyelonephritis and viral illnesses such as Hanta virus), (381, 542).

Vascular Damage-AKI from vascular damage occurs because injury to intrarenal vessels decreases renal perfusion and diminishes GFR. Causes of vascular injury include malignant hypertension, atheroembolic disease, preeclampsia/eclampsia, and hemolyticuremic syndrome (HUS)/thrombotic thrombocytopenia purpura (TTP) $(381,542)$.

\section{I.2. Epidemiology/Outcomes}

As mentioned previously, AKI has primarily become a nosocomial disease in the developed world. AKI has an incidence of 5-7\% in hospitalized patients $(93,390,562)$. This incidence appears to be rising over time $(214,582)$, which may reflect greater clinical vigilance and/or differences in how AKI is precisely defined. The etiology of AKI differs somewhat depending on if the onset of AKI occurs in the hospital (hospital-acquired) or prior to hospitalization (community-acquired). Depending on the study, prerenal etiologies account for $25-60 \%(73,213,317,318)$ and renal etiologies account for $35-70 \%$ of AKI cases $(73$, $93,213,317,318,363,389,562)$. Ischemic injury or nephrotoxins contribute to $80-90 \%$ of the renal etiologies $(213,317,318,363)$. Postrenal etiologies generally account for $<5 \%$ of AKI in hospitalized patients but this number increases to almost $20 \%$ in communityacquired cases (318). The disease burden of AKI results in an estimated $\$ 10$ billion in additional costs to the health care system in the Unites States (93) and is associated with a 
mortality of $45-70 \%(25,303,316,363,389,562,584)$. The diagnosis of AKI increases the risk of mortality 5.5 to 6.5 -fold as compared to a similarly ill patient without AKI $(93,303)$. Unfortunately, despite advances in knowledge of the pathophysiology of AKI, this figure has not significantly changed since the advent of dialytic therapy which is required in 20 $75 \%$ of patients $(93,94,303,317,318,384,389,562,584)$. There is burgeoning evidence for the direct negative impact that AKI has on distal vital organs (177). These distant organ effects may significantly contribute to the overall mortality observed in patients with AKI despite the initiation of dialytic therapy. For those patients surviving an episode of AKI requiring dialytic therapy, 10-50\% continue dialytic therapy after discharge $(93,94,227$, $303,317,318,336,384,389,477,562,584)$. Furthermore, patients that survive an episode of AKI requiring dialytic therapy and recover function are at increased risk of progressing to $\operatorname{ESRD}(9,227,336,584)$. In fact, it has been estimated that these patients make up 3\% of the overall yearly incidence of ESRD in North America $(583,584)$. Clearly, AKI has serious acute and chronic sequelae.

\section{I.3. Clinical Pathological Findings}

The term ATN is a misnomer. Indeed, the contradiction between the degree of histological abnormalities on human biopsy samples and the extent of GFR depression has served as a one of the main paradoxes in AKI. While the S3 segment of proximal tubule has been generally viewed as the most important site of injury in human $\operatorname{ATN}(56,137,410,514)$ the relative contribution of injury to the distal tubule that sits adjacent to the $\mathrm{S} 3$ segment in the outer medulla has been a point of controversy $(61,322)$. Settling this controversy is hampered by the relative lack of medullary tissue routinely obtained on clinical biopsies of AKI. Outside of rare frankly necrotic tubular cells that occur, the prominent findings in human biopsies include detachment of renal tubular epithelial cells from the basement membrane, sloughing of cells into the tubular lumen, effacement and loss of brush border in proximal tubular segments, and the formation of tubular casts derived from sloughed cells, tubular debris, and protein. While evidence of tubular injury is apparent, evidence of tubular cell regeneration can also been seen alongside the injury (514). Tubular cells with basophilic cytoplasm and hyperchromatic nuclei consistent with regenerating epithelial cells and even tubular cells undergoing active mitosis have been observed in many human biopsies of patients with ATN (514).

Alterations of the interstitium, vasculature, and glomeruli have also been documented in ATN. Interstitial edema is often observed and may develop from leakage of fluid from increased microvascular permeability or backleak of tubular filtrate into the interstium (511, 514). Peritubular accumulation of leukocytes in the interstitium has also been observed in experimental models of ATN $(168,627)$ although this does not appear to be a predominant feature in human ATN (514). Congestion of the peritubular capillaries in the outer medullary region is a salient feature of ATN that may play an important role in regional alterations of blood flow and exacerbating tubular injury during the extension phase. Additionally the accumulation of leukocytes in the vasa recta $(118,513,514,626)$ may also be an important contributor extending tubular injury. Changes in peritubular capillaries of the cortical region are less prominent but loss of some peritubular capillaries and dilatation of others has been described (276). While changes in arteries and arterioles are relatively subtle, biopsies of ATN have demonstrated vacuolization of smooth muscle cells as well as alteration of smooth muscle cell actin (285). As may be expected, the most noted glomerular alteration in ATN is collapse of the glomerular tuft due to hypoperfusion. Alterations in glomerular foot processes have also been described (516) but this has not been a consistent finding. 


\section{Pathophysiology, hemodynamics and inflammation}

\section{II.1. Phases of acute kidney injury due to Acute Tubular Necrosis}

As mentioned above, the term ATN correctly identifies the site of injury, though relatively few renal epithelial cells undergo frank necrosis. More commonly, sublethal changes in renal tubular epithelial cells are present and thus the term acute tubular injury may be more appropriate. Importantly, even sublethal changes can have a major impact on the decrement in GFR that is the hallmark of AKI.

Clinically, ATN and the associated decrease in GFR can be divided into initiation, extension, maintenance, and recovery phases. These clinical phases directly relate to cellular events that occur during the injury and recovery process (Figure 1). Although a clear mechanistic explanation between tubular injury and a fall in GFR has remained elusive, afferent arteriole vasoconstriction in response to tubuloglomerular feedback, backleak of glomerular filtrate, and tubular obstruction have all been postulated as mechanisms for decreased GFR in ATN (see below). All three of these mechanisms can be directly related to changes occurring in renal tubular epithelial cells.

The initiation phase of ATN occurs when renal blood flow (RBF) decreases to a level resulting in severe cellular ATP depletion that in turn leads to acute cell injury and dysfunction. Renal tubular epithelial cell injury is a key feature of the Initiation Phase (532). Renal ischemia in vivo rapidly induces a number of structural and functional alterations in renal proximal tubular epithelial cells that are directly related spatially and temporally with disruption of the normal framework of filamentous actin (F-actin) in the cell $(14,502,542)$ (Described in section III). The extent of these alterations depends upon the severity and duration of ischemic injury. Although these alterations usually fall short of being lethal to the cell, they do disrupt the ability of renal tubular epithelial cells and renal vascular endothelial cells to maintain normal renal function. Additionally, ischemic injury to vascular smooth muscles cells and endothelial cells during the initiation phase may also contribute to the structural abnormalities observed in the renal vasculature during ischemic AKI (285, $358,541)$.

Recent evidence now indicates that "activation" of epithelial and possibly endothelial cells during the early initiation phase results in the up regulation of a variety of chemokines and cytokines $(446,522)$ that are instrumental in initiating the inflammatory cascade (See below). The cellular localization of these phenomena within the kidney is just beginning to be elucidated (131). As these and other events resulting in cellular alterations are not well identified morphologically, they have been difficult to evaluate using standard methodologies. Therefore, what role these early cellular responses play in further worsening renal perfusion remains to be determined.

The extension phase is ushered in by two major events: continued hypoxia following the initial ischemic event and an inflammatory response (Figure 1). Both events are more pronounced in the corticomedullary junction (CMJ), or outer medullary region, of the kidney. Documentation of severely reduced blood flow, stasis and accumulation of RBC and WBC has been historically noted (See Figure 2), however, the epithelial ramifications of these events have only recently been uncovered $(329,446)$. It is during this phase that renal vascular endothelial cell damage likely plays a key role in the continued ischemia of the renal tubular epithelium, as well as, the inflammatory response observed with ischemic ARF. During this phase, cells continue to undergo injury and death with both necrosis and apoptosis being present predominantly in the outer medulla (257). In contrast, the proximal tubule cells in the outer cortex, where blood flow has returned to near normal levels, actually undergo cellular repair and improve morphologically during this phase. As cellular injury 
continues in the CMJ region during the extension phase, the GFR continues to fall. There is continued production and release of chemokines and cytokines that further enhance the inflammatory cascade (132). Interrupting the amplification of this inflammatory cascade may have therapeutic implications. Although the extension phase is probably the most promising phase for successful therapeutic intervention in ischemic ATN, there is a short window of opportunity. Based on animal models of renal ischemia, inflammatory cell infiltration in the outer medullary region of the kidney is significant by 24 hours following ischemia $(260,445,628)$ and leukocytes may begin to appear in as early as 2 hours after ischemia (606).

During the clinical phase known as maintenance, cells undergo repair, migration, apoptosis and proliferation in an attempt to reestablish and maintain cellular and tubule integrity (Figure 1). The GFR is stable albeit at a level determined by the severity of the initial event. This cellular repair and reorganization phase results in slowly improving cellular function and sets the stage for improvement in organ function. Blood flow returns toward normal and epithelial cells establish intracellular and intercellular homeostasis. During the recovery phase cellular differentiation continues, epithelial polarity is reestablished and normal cellular and organ function returns $(191,402,542)$. Thus, renal function can be directly related to the cycle of cell injury and recovery and this cell response to injury will be covered to greater extent later in this chapter.

\section{II.2. Hemodynamic changes}

Despite the role of tubular injury, the hallmark feature of AKI is a reduction in GFR, which by its essence implies an underlying impairment in hemodynamic regulation $(114,203,210$, $251,354)$ and indeed was originally characterized as "vasomotor" nephropathy (210).

Typically, the response is a sustained increase in renal vascular resistance (RVR) that may be attributable to a number of factors $(62,424,495,532)$. Renal blood flow is determined by a balance between factors that promote either vasoconstriction or vasodilation. The large number of potential contributors make it difficult to pinpoint a single cause for the increase in RVR. Rather, increased RVR is likely related to a number of variables: 1) alteration of intrinsic mechanisms of constriction; 2) enhanced production of vasoconstrictors or decreased production of vasodilators; 3 ) the altered response to vasoconstrictors or vasodilators.

II.2.1. Tubuloglomerular feedback and adenosine-Sublethal tubular damage results in impaired proximal sodium reabsorption (See Section III). This impaired tubular transport of sodium is expected to activate tubular glomerular feedback (TGF) mechanisms leading to increased afferent vascular resistance and a concomitant decrease in GFR. Studies by Blantz's group in the 1980s using micropuncture techniques demonstrated that uranyl nitrate nephrotoxicity reduced SNGFR and that inhibition of the TGF-mechanisms by furosemide, which inhibits solute reabsorption in the TAL, improved SNGFR (434). This observation is not uniform among different models of injury as furosemide was not able to protect functional changes in SNGFR in ischemic kidney injury (53) and in general furosemide has not been demonstrated to be of clinical utility in altering the course of human AKI (206). However, these studies are complicated by the fact that furosemide has a number of potential kidney effects in AKI. Although additional studies in animals (7) and humans (6) have provided evidence for the importance of TGF in AKI, the extent that TGF contributes to diminished GFR in clinical AKI is not fully elucidated.

Adenosine, a potent renal vasoconstrictor resulting from the catabolism of adenine nucleotides, mediates the TGF response via the activity of A1 receptors (528). In the setting of renal injury, adenosine activity may also lead to increased renal vasoconstriction. Some 
studies support the idea that adenosine inhibition may prevent vasoconstriction and preserve injury in the setting of $\mathrm{I} / \mathrm{R}$. In rats, administration of the non-selective adenosine receptor antagonist theophylline preserved renal blood flow when administered before ischemic injury and increased renal blood flow after 5 days of recovery (326). In addition, theophylline was shown to preserve GFR in patients following exposure to a contrast agent relative to a placebo group (142).

Although encouraging, other studies from animal models raise questions about the nature of adenosine activity in the setting of injury. For example, transgenic mice lacking A1 receptors were unexpectedly more sensitive to ischemia reperfusion injury (528). In addition, increasing adenosine by inhibiting adenosine deaminase lessens the severity of ischemic injury (64). The protective effects may be associated with A2a receptor activity (409) and is likely mediated via effects on inflammatory cells (287). Thus, it appears that adenosine may be involved in multiple aspects of acute kidney injury and therapies geared toward targeting TGF and other hemodynamic responses still warrant additional consideration.

II.2.2. Sympathetic Nervous System, Norepinephrine and Ang II-Renal vascular resistance during AKI may be enhanced by increased sympathetic nervous activity. Reductions in effective circulating plasma volume associated with hypotension would necessarily lead to an increase in activation of the sympathetic nervous system. Increase sympathetic activity is highly characteristic in rhabdomyolysis (67). Conger demonstrated that direct infusion of norepinephrine into the renal artery for periods of up to 60 minutes in rats could lead to prolonged reductions in RBF resulting in AKI (99). There are elevated levels of norepinephrine in the renal venous blood following ischemic renal injury (156). In addition, denervation of the kidneys improves RBF and attenuates the subsequent manifestation of acute tubular necrosis in ischemia and endotoxin models of AKI (156, 472, 588). Pharmacological blockade with clonidine, propranolol or guanethidine improves RBF in rat ischemia/reperfusion (I/R) injury (512). Recently, treatment with agmatine was shown to reduce SNS activity and ameliorated the resultant injury due to ischemia/reperfusion injury (527).

Increased sympathetic nerve activity is also a potent stimulus for renin secretion. Ang II, a potent renal vasoconstrictor, may also contribute to decreased blood flow in AKI. Patients with AKI have elevated plasma renin activity and hypertrophy of the granular cells of the juxtaglomerular apparatus (521). It is interesting to note that renal vasoconstriction to Ang II and NE are augmented by adenosine A1 activity (4). Despite this, there seems to be little evidence to support a role for Ang II activity in AKI; In fact, blockade of Ang II with either receptor blockers or ACE inhibitors increases the risk of AKI in patients following cardiac surgery (12).

II.2.3. Endothelin-Endothelin is a potent renal vasoconstrictor and produces profound reductions in GFR (149). Endothelin conveys its effects via at least two distinct receptor subtypes, the ET-A and ET-B receptors. Interestingly, activation of each of these subtypes has the potential to reduce GFR. In anesthetized rats, the selective ETA antagonist, A-127722 inhibited afferent arteriolar constriction, while specific activation of the ETB resulted in both afferent constriction as well as efferent vasodilation (226).

In humans, there is compelling evidence that the endothelin system may be activated in the setting of acute injury. Increased endothelin levels have been reported in patients with AKI due to severe sepsis (436). In a rat model of cold ischemic storage and transplant, pre-proendothelin mRNA was upregulated and immunoreactive ET-1 localized in the peritubular capillary endothelial cells following reperfusion injury (605). The role of endothelin in AKI 
is supported mainly by pharmacological studies carried out in models demonstrating modest protective effects. For example, both non selective and ET-A specific antagonists have been shown to protect rats from the manifestion of renal I/R injury $(83,215,277)$. Gellia et al, demonstrated a significant protective effect of ET-A blockade using and extreme model of IR injury in which $100 \%$ of control animals died within 3 days, while there was $75 \%$ survival over 2 weeks in animals treated with the ETA antagonist (165). However, the effect of ET antagonism in humans is less clear, as it did not manifest any protection prophactically for contrast induced nephropathy (586).

II.2.4. Prostaglandins-Arachidonic acid released from phospholipids is converted by cyclooxygenase (COX-1 constitutive, COX-2 inducible) in the kidney to $\mathrm{PGI}_{2}, \mathrm{PGE}_{2}, \mathrm{PGF}_{2}$, $\mathrm{PGD}_{2}$, and thromboxane $\mathrm{A}_{2}$. $\mathrm{PGE}_{2}$ and $\mathrm{PGF}_{2}$ are produced predominantly but not exclusively in the renal medulla, whereas degradative enzymes are present in both the cortex and medulla (192). During renal hypoperfusion, the intrarenal generation of vasodilatory prostaglandins, including prostacyclin $\left(\mathrm{PGE}_{2}\right)$ promotes the maintenance of renal perfusion.

Cox-2 inhibitors represent a major cause of drug-induced AKI and their use can increase risk for AKI following surgery or administration of radiocontrast agents $(66,70)$. Inhibitors of COX-2 exacerbate reductions in GFR and medullary blood flow in endotoxemia induced by LPS (590). Conversely, iloprost, the PGI analog, preserved RBF and corticomedullary hypoxia in a similar endotoxemic model (238). Iloprost can ameliorate the degree of renal insufficiency and renal blood flow in response in ischemia reperfusion in dogs (548). These effects are likely to be mediated by vasodilation, however, iloprost also decreases ICAM expression on the endothelium (224). In one study, prostacyclin protective effects were reported to be independent of effects on renal blood flow (148). Recently, some reports indicate that PG analogs may be a useful prophylaxis in preventing AKI clinically (518).

II.2.5. Platelet activated factor-Platelet activated factor (PAF: 1-O-alkyl-2-acetyl-snclyeryl phosphocholine) is a lipid mediator with a wide spectrum of biological activity. It can be produced by a number of different cells types including kidney cells. Its effects on renal function include reductions in GFR and RBF, making it a possible candidate for involvement in the development of acute kidney injury (337). Its hemodynamic effects occur by directly stimulating afferent arteriolar constriction as well as mesangial cell activity influencing $\mathrm{Kf}$, and perhaps additional activity related to vascular obstruction (inflammation, vide infra) and cell toxicity (337). Acute renal injury may activate PAF via $\mathrm{Ca}++$-dependent phospholipase $\mathrm{A}_{2}$; PLA 2 acts on membrane 1-O-alkyl-2-

acylglycerophosphocholine to generate the precursor Lyso-PAF and subsequent conversion to PAF is mediated by an acyl transferase. Renal synthesis of PAF occurs following injury to the kidney in diverse models of AKI (e.g., ischemia, glycerol, gentamicin, endotoxemia) and treatment with PAF antagonists ameliorates the severity of AKI (337).

II.2.6. Alterations in vascular function secondary to injury-In addition to the induction of vasoactive factors, renal injury may directly damage the renal vasculature and alter its activity; such damage may influence vascular responsiveness, barrier function, coagulation cascades and/or inflammatory processes $(74,532,610)$.

Studies by Conger and colleagues were among the first to highlight alterations in vascular responsiveness following $\mathrm{AKI}$; these investigators demonstrated that post-ischemic rat kidneys do not autoregulate blood flow, and in fact, manifest vasoconstriction in response to decreased renal perfusion pressure. This occurred even one week following recovery from I/ $\mathrm{R}$ when total renal blood flow had returned to baseline values (100). Morphological evidence of endothelial and smooth muscle injury was present up to 7 days following I/R 
induced by renal artery clamping or norepinephrine infusion. The increased constrictor responses could be blocked by $\mathrm{Ca}++$ antagonists (101).

NOS3, (endothelial NOS) is produced at high levels particularly in the renal medulla (359) and its activity may represent an important mechanism to preserve medullary blood flow in response to renal vasoconstrictors such as Ang II (648). However, following renal injury, there is impaired endothelial NOS function, demonstrated by a loss of vasodilator responses to acetylcholine and bradykinin (101). A role for NOS3 in preservation of flow is suggested by the greater degree of renal damage that occurs in NOS3 knockout mice in response to endotoxin (589). Several studies using animal models have been utilized with the goal of increasing renal NO activity and/or preserving endothelial function. For example, administration of L-arginine, the NO donor molsidomine, or the eNOS cofactor tetrohydroboipterin can preserve renal vascular perfusion and attenuate AKI induced by $\mathrm{I} / \mathrm{R}$ $(112,161,244,483,485,525)$.

Recent studies have focused on rapid changes in endothelial function and their potential role in the initiation or maintenance of injury in the setting of I/R. Yamamoto et al., reported that renal perfusion in peritubular vessels was compromised within minutes of unclamping, characterized by sluggish and occasionally retrograde blood flow (610). Infusion of endothelial cells to athymic nude rats, or other non-endothelial cells harboring the NOS3 gene expression construct, protected against this early compromise in blood flow (74). Arrerio et al. propagated endothelial like cells from mesenchymal stem cells; these cells expressed markers typical of endothelial cells such as Tie-2, flt, flk and NOS3.

Administration of these cells to control rats generated short-term engraftment into the vasculature and short term protection from I/R injury (13). Taken together, these data suggest that endothelial function, particulary via NOS3 activity, has protective effects on the severity of AKI.

Reactive oxygen species may influence the effects of vasocontrictors and vasodilators and lead to an increase in renal vascular resistance. Increased renal superoxide production augments renal vasoconstrictor responses particularly in the renal medulla. Superoxide promotes vasoconstriction and enhances the reactivity of Ang II in the renal cortex and medulla (647). Oxidative stress in the kidney also enhances the vasoconstrictor effects of adenosine (91). Superoxide, by virtue of its known activity to convert NO to peroxynitrite, may block the normal homeostatic mechanism maintaining medullary perfusion and these responses may be normalized with the use of antioxidants (647).

\section{II.2.7. Other factors affecting renal blood flow}

II.2.7.1. Cell swelling and Edema: As described below in Section III, alterations in cell energetics leads to alterations in cell shape including swelling induced by enhanced sodium uptake. In addition to potentially affecting cell death, increased cell swelling may increase extravascular pressures on the peritubular capillaries and restrict flow. Flores et al demonstrated that when cellular swelling was reduced by infusion of mannitol, there was an improvement in renal blood flow (152). Tubular obstruction by cellular debris may influence hemodynamic response, In models of direct obstruction by ureteral clamping, there is rapid impairment of renal blood flow and this may be due alterations in tubular pressure and/or alterations in hemodynamic factors liberated secondary to injury $(200,222,234,500,581)$.

$\mathrm{I} / \mathrm{R}$ injury in rats alters cytoskeletal organization of small arterioles and endothelial cells $(285,532,534)$. These alterations contribute to the breakdown of VE-cadherin in and the integrity of the tight junctions of peritubular capillaries as evidenced by leakiness of high molecular weight dextrans (>300,000 Da) into the interstitial space (534). Breakdown of barrier function may be the result of the activation of MMP-2 or 9 (533). The potential 
ramifications of endothelial leakiness on the course of AKI are unclear. Increased edema resulting from leakiness may compromise renal perfusion by compressing peritubular capillaries and/or increasing hemoconcentration and exacerbating RBC trapping (201-203, 532).

II.2.7.2. Vascular injury and thrombosis: Although not well explored, it worth mentioning that endothelial damage in I/R injury may also promote thrombosis and fibrin deposition. The formation of microthrombi have been described in renal IR models as well as in renal transplant biopsies $(141,532)$. Evidence that endothelial cell damage promotes thrombus formation in renal $\mathrm{I} / \mathrm{R}$ is minimal and its potential role is suggested mostly from work in other organ systems. Potential microthrombus formation may be associated with loss of surface inhibitory factors, notably thrombomodulin, which allows fibrin formation by disinhibiting thrombin (532). Recently, a soluble thrombomodulin molecule was shown to attenuate the degree of renal injury following ischemia in rats; this was associated with an rapid effect on preservation of perfusion within renal capillaries and decreased leukocyte adhesion (492).

In addition, tissue factor, a membranous glycoprotein that functions as a receptor for the coagulation system and is normally not present on quiescent endothelial cells, was shown to be elevated in peritubular capillary endothelial cells following renal I/R injury. Inhibition by antisense oligonucleotides manifested a significant protection indicated by preservation of overall morphology and mortality in rats (357).

Endothelial cells may also modulate the injury process via binding of activated serum protein $\mathrm{C}$, to the endothelial protein $\mathrm{C}$ receptor. Activated protein $\mathrm{C}$ exerts both antithrombotic and cytoprotective properties, the latter via modulation of protease-activated receptor-1 (PAR-1) signaling (240). In a recent study, a mutant APC molecule that retained PAR agonist function preserved renal function in AKI, but a mutant APC that retained only anti thrombogenic capacity did not protect against LPS injury (492). In clinical trial of human sepsis patients, Drotrecogin (a recombinant human activated protein C) significantly decreased $28 \mathrm{~d}$ mortality over the placebo group(48).

\section{II.2.8. Lack of vasodilator response and role of infiltrating cells and renal}

injury-The data on animal models suggest that vasoconstriction contributes to the early loss of GFR and triggers injury. While reductions in RBF may represent an important feature in the initiation phase of AKI, increasing RBF after established injury may not resolve GFR. Figure 3 illustrates proposed pathways in which factors independent of direct vasoconstriction may ultimately impact the loss of renal blood flow and GFR in AKI

Numerous examples in the literature demonstrate a lack of effect of vasoactive factors at correcting established AKI. Perhaps the most well known example of this is represented by the use of "renal-dose" dopamine. At low doses (i.e., $3 \mu \mathrm{g} / \mathrm{kg} / \mathrm{min}$ ) dopamine is vasodilatory and promotes diuresis primarily through the D-1 receptor and has beneficial effects in multiple models of AKI (121). Despite this, multiple studies have consistently demonstrated that renal-dose dopamine or the dopamine agonist fenoldapam, do not influence outcome of AKI in patients despite producing diuresis $(45,254,255,288,560)$. Similarly, atrial natriuretic peptide can produce diuresis in AKI patients but does not provide any improvement in renal function or recovery (305).

Characteristic of renal injury is the prominent infiltration of leukocytes, primarily neutrophils occurring fairly rapidly following ischemia/reperfusion injury in rodents (160, $375,628)$. Leukocyte adhesion to activated endothelial cells following $I / R$, and subsequent red blood cell congestion results in a further compromise renal function by influencing renal 
hemodynamics. Leukocyte adhesion is proposed to impair renal function in AKI in the following ways: 1) Leukocytes may physically impair capillary flow, particularly in the renal medulla and exacerbate hypoxia in this region; 2) Leukocytes may generate molecules exacerbating vasoconstriction, such as ROS and/or vasoactive lipids; 3) Leukocytes may contribute to parenchymal cell injury, the result of which may be to increase tubular lumen pressure and reduce GFR. Leukocyte induced interstitial edema can increase interstitial pressures and retard peritubular capillary flow. These activities may represent a switch to the extension phases of AKI and be the cause of resistance to vasodilator therapy after the initiation of AKI. To our knowledge, there have been no studies correlating the adhesion of leukocytes with reductions in medullary blood flow and subsequent resistance to vasodilator therapy.

\section{II.3. Inflammatory process in AKI}

In addition to the myriad of altered vascular functions that may influence the development of AKI, inflammation is mediated in part by the adhesion of leukocytes to affected endothelial cells. Inflammatory cell infiltration is a prominent early feature in the outer medullary vasa recta capillaries following injury. There is an increase surface expression of leukocyte adhesion molecules ICAM-1 and P and E-selectin on endothelial cells in response to injury $(158,261,383)$. In vivo imaging studies have shown leukocyte adhesiveness to the peritubular capillary wall occurs within hours of reperfusion $(259,492)$. Treatments geared toward reducing endothelial/leukocyte interactions by targeting these endothelial adhesion molecules preserve blood flow and protects against renal damage in IR induced AKI (158, 261, 383). The endothelium may also be the source of chemoattractant factors, such as fractalkine $\left(\mathrm{CX}_{3} \mathrm{CL} 1\right)$, which is expressed following renal injury and may promote infiltration of macrophages (see below).

On balance, most studies in animal models confirm an important role of inflammation in the establishment of AKI. Studies geared to prevent neutrophil infiltration by interfering with PSelectin, ICAM/CD18 pathways, mitigate the degree of renal damage in ischemia reperfusion injury $(160,262,395,443-445)$. Both anti-oxidant therapy and minocycline treatment reduced the extent of leukocyte infiltration into post-ischemic kidneys, an effect that was partially attributable to inhibition of ICAM-1 expression $(259,330)$. Neutrophil extravasation has been shown to be dependent on the activity of the adenosine A2A receptor which minimizes the degree of parenchymal injury (57). In human AKI, ICAM antibody pretreatment protected against delayed graft function in transplant recipients relative to a group of recipients receiving the contralateral kidneys from the same donors. Interestingly, immunoneutralization following transplant did not affect the development of DGF (155, 195, 473). Thus, this treatment may prevent injury, but may be ineffective after initiation of injury.

The degree to which neutrophils actually contribute to renal injury remains somewhat unclear. Neutrophils are not prominent in biopsies of patients with acute kidney injury (155). There are conflicting reports regarding the effect of neutrophil depletion on I/R induced AKI with some studies demonstrating a protective effect while others demonstrating no effect $(143,262,444)$. DeBroe's group suggested that the degree of neutrophil accumulation following injury may be overestimated, since traditional neutrophil markers (MPO and chloroacetate esterase) cross react with macrophages (628).

II.3.1. T cells-Recent evidence suggests that monocytes, primarily T-cells, may play an important role in AKI. T-cells are apparent in the medullary vasa recta in animal models of AKI and are present in biopsies of patients with AKI (Figure 4) (155, 627). It is noteworthy that blockade of CD18a/ICAM pathway, thought to be affecting neutrophils, also affects the 
deposition of T-cells (78). Evidence supporting an important role for T-cells derives from studies in which T-cells are depleted using either antibody neutralizing approaches (624) or genetic approaches (117) reducing the degree of renal injury to I/R. De Greef and colleagues further demonstrated the importance of the early expression endothelial B7-1 in peritubular capillary endothelial cells on the adherence of $\mathrm{T}$ cells and the manifestion of injury following I/R (Figure 4) $(118,537)$. It remains unclear how T cell deposition contributes to the injury process. However, evidence suggests that there is an important role for T-cell derived IFN- $\gamma$ since reconstitution studies demonstrate that $\mathrm{CD} 4+\mathrm{T}$ cells derived IFN- $\gamma$ null mice are not sufficient to recapitulate the injury observed in T-cell replete animals (117).

Conversely, regulatory T cells (Tregs, CD4, CD25, Foxp3) are lymphocytes with immunosuppressive properties that may traffic to areas of inflammation and mitigate immune reactions. Depletion of Tregs with anti CD25 mAb potentiates kidney damage induced by IRI, and is associated with increased neutrophils, macrophage and cytokine responses. When adoptive transfer of T cells from Foxp3 null mice was carried out, renal injury is worsened, while adoptive transfer of Treg alone into $\mathrm{T}$ cell deficient mice results in significant protection (274).

A potential problem in understanding the pathological role of $\mathrm{T}$ cells in the mediation of AKI is that CD4+ cells are classically thought to play a role in antigen specific adaptive immunity that requires $2-4$ days for processing. Such activity is inconsistent with a role for these cells in AKI. However, natural killer T (NKT) cells, which express the TCR (T cell receptor) and the marker NK1.1 represent a unique and small subset of CD4+ cells. These cells can produce large amounts of cytokines such as IFN $\gamma$ and TNF (Th1 type), or IL-4 and IL13 (Th2 type). NKT producing IFN $\gamma$ are present in the post-IR kidney within 3 hours of injury (311). Blockade of NKT cell activation with the anti-CD1d mAb, NKT cell depletion with an anti-NK1.1 mAb in wild-type mice, or use of $i$ NKT cell deficient mice (Ja18-/-) inhibit the accumulation of IFN- $\gamma$-producing neutrophils after IRI and prevented AKI (311), suggesting that NKT cells are the major early acting CD4+ population acting affecting kidney injury following $\mathrm{I} / \mathrm{R}$.

II.3.2. Macrophages-Macrophages are derived from monocytes and have phagocytic capabilities. Macrophages infiltrate the injured kidney within 1 hour of ischemia reperfusion; this activity is mediated by fractalkine ( $\left.\mathrm{CX}_{3} \mathrm{CL} 1\right)$, which is expressed by injured endothelial cells and is a potent chemoattractant and adhesion molecule for ( $\mathrm{CX}_{3} \mathrm{CL} 1$ receptors) $\mathrm{CX}_{3} \mathrm{CR} 1$ and $\mathrm{CCR} 2$ expressed on monocytes and macrophages. Both ischemia and cisplatin AKI induced fractalkine expression on peritubular capillary endothelial cells. Blockade of this pathway with CX3C receptor-1 antibody effectively reduces the severity AKI in mice while adoptive transfer of activated RAW 264.7 macrophages restores sensitivity to injury $(312,407)$. Macrophages lacking CCR do not infiltrate injured kidneys and the resultant injury is less severe (312). Infiltrating macrophages may exacerbate injury by producing a high levels of the cytokines IL-1a, IL-6, IL 12 and TNF-a and promoting further inflammation (272).

The last several years has seen an increased awareness of the diversity of macrophage phenotypes. Within the setting of kidney injury, macrophages are most abundant during the repair response suggesting a beneficial role for macrophages in recovery. A distinct population of macrophages, referred to as M2, secrete anti inflammatory compounds such as IL-10 and TGF- $\beta$ and may be involved in tissue repair by secreting potential trophic growth and angiogenic factors $(136,591)$. Proof of principal for this activity was demonstrated by Want et al., in which ex vivo programming of macrophages toward an M2 phenotype ameliorates chronic renal inflammation (593). Jang et al. found impaired recovery from kidney IRI when macrophages were depleted following kidney IRI using liposomal 
clodronate (231). Administration of macrophages during the repair phase of ischemic injury increased renal cell proliferation and enhanced recovery (577). In one study, macrophages were ablated using a transgenic approach in which the diphtheria toxin (DT) receptor was expressed on CD11b-positive cells. DT treatment of these mice reduced macrophages and resulted in prolonged and less successful recovery from AKI (327). The reparative potential of macrophages may be mediated by Wnt ligands expressed on the surface of regenerative macrophages, and interact with epithelial cells expressing Wnt-receptors (327).

II.3.3. Dendritic cells-CD11c+ MHC class II+ DCs are the most abundant leukocyte subset in the normal mouse kidney suggesting an important role in renal immunity and inflammation. When activated due to injury, these cells may release pro-inflammatory factors and interact with NKT cells. However, the role of dendritic cells in AKI is not yet clear. While dendritic cell activation has been suggested to lead TNF-a production in the setting of ischemic AKI (129), there have been few functional studies on dendritic cell activity in the injury process. In one recent study, ablation of CD11c DC using a DT mediated approach, increased in sensitivity to cisplatin induced injury, suggesting that DC have a protective role via an unknown mechanism (536).

II.3.4. Inflammatory signals in AKI—In combination with endothelial adhesiveness, inflammatory mediators are synthesized and released by both tubular epithelial cells, and activated leukocytes. Tubular epithelial cells produce TNF-a, IL-1, IL-6, IL-8, TGF- $\beta$, MCP-1, ENA78, RANTES an fractalkines, while leukocytes may produce IL-1, IL8, MCP-1, reactive oxygen species and eicosanoids. These factors act in concert to promote inflammation in a positive feedback loop promoting further kidney injury (63). The control of inflammation and the various factors that regulate it is substantial and the subject of numerous reviews. A limited consideration of some of the major pathways is described below.

For example, IL1 $\beta$ is a chemoattractant that can recruit leukocytes to areas of injury; pro IL1 $\beta$ is processed by caspase 1 (ICE) to form mature IL1 $\beta$. IL1 $\beta$ levels are increased in mice following ischemia reperfusion injury (262). Based on studies using receptor antagonists, or IL-1 $\beta$ null mice, the blockade of IL- $1 \beta$ reduces the extent of neutrophil infiltration following ischemic injury, but has no effect on the resultant loss of renal function (190). Caspase-1 is also involved in the activation of IL18. IL-18 is enhanced in AKI and may represent an early biomarker of acute injury (77). Conversion to mature IL-18 is not observed in caspase-1 $1^{-1-}$ mice, relative to wild type controls, and caspase 1-/- are protected from ischemic injury. Similarly, the caspase inhibitor Quinoline-Val-Asp(Ome)- $\mathrm{CH}_{2}-\mathrm{OPH}(\mathrm{OPH}-001)$ also prevented IL-18 processing while IL-18 immunoneutralizing antibodies preserved renal function following $\mathrm{I} / \mathrm{R}(365,366)$.

IL-6 may also promote inflammation in AKI; injured proximal tubules produce molecules that activate the expression of IL-6 expression on infiltrating macrophages and IL6 is found at elevated levels in the serum of mice following renal injury (266). IL-6 knockout mice are resistant to AKI, but sensitivity to injury is restored by adoptive transfer of wild-type macrophages (266). However, there are mixed data on the importance of IL6 in AKI , as IL-6 knockout mice are more sensitive to injury induced by cisplatin, possibly via an increase in pro-apoptotic regulatory signals (374).

Toll like receptors (TLR) are a family of pattern recognition receptors that detect motifs on pathogens and host material important in the activation of innate immunity. Located primarily on immune cells, TLR signaling transduces microbe host interactions into an inflammatory state which is required to eliminate invading organisms; when uncontrolled TLR signaling can lead to tissue damage (5). TLRs mediate their pro-inflammatory 
responses by a common downstream signaling molecule, MyD88, which leads to the activation of NFK-B inflammatory signaling.

TLR 4 is the classic endotoxin receptor. $\mathrm{C} 3 \mathrm{H} / \mathrm{HeJ}$ mice lack the TLR4 receptor, while $\mathrm{C} 3 \mathrm{H} /$ $\mathrm{JeOuJ}$ mice with intact TLR4 receptors manifest an increased response to endotoxin with greater production of the pro-inflammatory cytokine TNF-a, more pronounced renal damage and neutrophil infiltration when compared to $\mathrm{C} 3 \mathrm{H} / \mathrm{HeJ}(108)$. The influence of specific TLR may be dependent on the model of sepsis. Using a cecal ligation puncture (CLP) model of sepsis, TLR4 and TL2 deficient mice were not protected against renal damage and inflammation, while MyD88 knockout mice are resistant (120). TLR 9 may mediate CLP induced renal inflammation because severity of AKI is reduced in both TLR9 knockout mice and following the administration of chloroquine, an antagonist of TLR 3, 7,8, and 9(619).

TLRs also interact with non microbial endogenous substances, which has expanded this field beyond sepsis into conditions of autoimmunity and inflammation (457). For example, HMBG-1 proteins, potentially released from damaged cells, can activate inflammatory signaling in human renal proximal tubules and endothelial cells from wild type mice, but not from TLR 4 knockout mice $(90,280)$. There is a blunted induction of cytokines and chemokines in TLR4 and TLR2 null mice, as well as reduced neutrophil and macrophage infiltration in response to ischemia and other models of injury, such as cisplatin (113, 295, $609,638)$. TLR4 expression is found on vasa recta capillaries within 4 hours of ischemia reperfusion injury; the expression of adhesion molecules CD54 and CD62E in peritubular capillaries was not observed in TLR4 null mice compared to wild type. Secondary increases in TLR4 on proximal tubule cells occur within 24 hours of injury and may amplify the proinflammatory state of I/R injury (90). Recent evidence suggests that the TLR 4 pathway may be important in human AKI in the setting of transplant. Donor kidneys with a TLR4 loss-offunction allele contained less TNFa, less MCP-1, more heme oxygenase 1 (HO-1), and exhibited a higher rate of immediate graft function (280).

Another pro-inflammatory regulatory system that can influence acute kidney injury is the complement (C) system. The $\mathrm{C}$ system contains three activation pathways that participate in innate immunity (441). Recent studies have suggested that the alternate complement pathway may be active in the setting of acute kidney injury by ischemia reperfusion (643). The complement system is proposed to represent a primary regulator of neutrophil infiltration. Mice deficient in complement factor B (fB-/-), an essential component of the alternative pathway, develop substantially less functional and morphologic injury, less tubulointerstitial complement $\mathrm{C} 3$ deposition, and less neutrophil infiltration in the outer medulla after I/R, relative to wild type controls (546). Complement processing in AKI may be controlled by the complement inhibitor Crry, which redistributes from the basolateral membrane in response to I/R (547). Evidence in support of this activity derives from studies in which Crry deficient mice demonstrate an increased sensitivity to IR injury (547). Surprisingly, despite a significant increase in C3 generation following endotoxemic (LPSinduced) kidney injury, blockade of the complement cascade does not protect against kidney injury in this model (107). Both C5a and C5b-9 contribute to renal injury independent of neutrophil attraction, perhaps by induction of apoptosis and impairing recovery responses following IR $(441,488)$. The activation of complement system is required for the production of macrophage inflammatory factor -2 and keratinocyte derived chemokine (KC) in renal tubule cells following I/R in an NF- $\kappa \mathrm{B}$ dependent fashion (545).

In summary, the general observations described above suggest a complex interplay with various pro-inflammatory signals. The sources of these various signals and how they are regulated at the molecular level are only beginning to be understood. For example, the regulation of pro-inflammatory genes may be secondary to the activation of upstream 
transcriptional activators of inflammatory genes, such as interferon regulatory factor-1 (IRF-1). In proximal tubule cells, IRF-1 expression is controlled by ROS activity in vitro, and it is produced rapidly by outer medullary tubular epithelial cells following renal injury. Null mutations in the IRF-1 gene reduce the degree of inflammation and pro-inflammatory gene expression following ischemic AKI (592). As mentioned, initial increases in endothelial TLR4 expression may be required for subsequent adjacent tubular TLR4 activity serving to amplify pro-inflammatory mechanisms. Future studies will likely be geared toward deciphering the complex interacting network of pro-inflammatory interactions that may aid in developing potential therapeutic targets.

\section{II.4 What is the link between tubular injury and GFR?}

A loss of GFR necessarily indicates that Starling forces are impaired during the injury process that results in renal dysfunction. Although the above sections describe a number of parameters that have been proposed (vasoconstriction, tubular congestion, edema, inflammation), no single factor has been demonstrated to be responsible for the entirety of the reduced GFR in response to tubular injury. Rather, the more likely interpretation from the multitude of these studies is the following; 1) Early vasoconstriction observed in many models impairs blood flow and GFR and is likely mediated by a number of redundant pathways. As such, blockade of any one pathway is not likely to produce significant benefits. 2) Features secondary to inflammation or structural changes in the renal parenchyma likely sustain reductions in GFR. At this point, hemodynamic therapies may serve a more limited role and the therapies should be directed toward blocking inflammatory pathways and/or restoring structure. 3) A given therapy may be effective in some settings, but depends on the timing. Therefore, a greater understanding of the phases of injury and methods to evaluate such phases will play an important role in designing effective treatments.

\section{II.5. Remote effects of $\mathrm{AKI}$ and possible causes for mortality}

In addition to altering kidney function, renal tubular damage may set off a series of events that alter physiological responses in distant organs and that may play a significant role in mortality. AKI increases the risk of death in patients with other co-morbid conditions and death per se may not be due to loss of renal function (237). Experimental models of renal ischemia reperfusion lead to increased pulmonary vascular permeability and macrophage infiltration (279). Rabb's group has also shown that renal I/R increases the permeability of the blood brain barrier (335), induces histological evidence of damage in neurons and microglial cells, and increases levels of pro-inflammatory cytokines such as G-CSF in the cerebral cortex and corpus collosum. Post AKI mice performed poorly in an open field locomotor tests to evaluate brain function (335). With regard to cardiovascular function, Kelly et al demonstrated decreased cardiac contractility and cardiac myocyte apoptosis in mice following I/R injury (256).

Based on these observations a developing view is that kidney injury sets in motion a complex systemic inflammatory response that is the basis for organ cross talk and it is likely that liberated pro-inflammatory cytokines (177). In kidney ischemia models in mice, there is evidence for the distinct activation of various circulating cytokines including $\mathrm{KC}, \mathrm{G} \mathrm{CSF}$, IL-6, IL-1 $\beta$ and IL-12 (177). An important potential area of future investigation will be to determine if treating these cytokine responses influence renal as well as the function of distant organs and influence mortality.

The idea that loss of GFR per se is not the single critical feature of mortality risk in AKI was addressed by Humes and colleagues. These investigators pioneered the bioartificial kidney which consists of a hemofilter in a continuous venovenous hemofiltration circuit (CVVH) 
with a cartridge containing renal proximal tubule cells, also called the Renal Tubule Assist Device (RAD). In nephroctomized dogs treated with endotoxin to simulate sepsis, the RAD helped to reduce overall inflammatory cytokine levels and improved cardiovascular parameters relative to dogs that were similarly dialyzed but without the RAD (150). Consequently, therapeutic strategies that target solute clearance, as well as, remote mediators of inflammation may hold future promise for addressing the full clinical impact of AKI.

\section{Cellular response to injury}

\section{III.1. Energetics and structure}

As described above, AKI is characterized not only by cell death, but also by a large spectrum of cellular injury (573), which may lead to the initiation of hemodynamic and inflammatory pathways reducing kidney function (Figure 2, 3 and 4). Therefore, an understanding of the cellular responses leading to both lethal and sublethal injury represents an important element in understanding the underlying pathophysiology of AKI. The varying sensitivity of renal cells to injury results from physiological parameters including the ratio of the energetic substrate to metabolic demand, the physical location of the cells within the kidney, the degree of regional blood perfusion in the aftermath of the insult, oxygenation status and membrane permeability.

III.1.1. Energetics and sensitivity to injury-In most animal models of AKI, the proximal tubule is considered the most sensitive cellular entity to ischemic, hypoxic or nephrotoxic damage $(62,68)$. However, the thick ascending limb of Henle is also highly metabolically active and is dramatically affected is certain models of AKI (61). In a rat model of ischemia reperfusion, early morphological alterations (such as tubular dilatation and loss of renal microvilli) are observed in all proximal tubule segments within hours of the initiation of reperfusion $(489,573)$. Proximal tubule cells within the S1 and S2 segment manifest a largely reversible injury; if cell death occurs, it localized primarily in the S3 segment (573).

Proximal tubule sensitivity relates largely to high metabolic rate and a strong dependence on oxidative phosphorylation. The intact mammalian kidney reabsorbs nearly $80 \mathrm{meq} \mathrm{Na} / \mathrm{g}$ kidney/day across the renal tubules and accounts for nearly $70 \%$ of oxygen utilization by the kidney (306). To meet this demand, tubule cells generate a significant amount of ATP. The preferential energy substrate is non-esterified free fatty acids, primarily palmitate, and to a lesser degree, lactate, citrate and pyruvate (306). Proximal tubules do not utilize glucose, but rather are gluconeogenic (306).

Because of its limited glycolytic capacity, renal ATP concentrations fall to 20\% of control values within $10 \mathrm{~min}$ of renal artery occlusion $(265,578)$. It is interesting that cortical values drop much more dramatically than medullary levels reflecting the higher proportion of proximal tubule cells in the cortex. In addition, a proportion of medullary cells are capable of anaerobic glycolysis. Medullary collecting duct cells appear largely resistant to ischemia and these cells can maintain ATP levels at approximately $60 \%$ of control values when oxidative phosphorylation is blocked and can even increase their glycolytic capacity under stress conditions (24). In contrast, both the medullary thick ascending limb and S3 proximal tubule cells have a limited glycolytic capacity and are unable to sustain normal ATP levels in the face of oxidative stress $(42,561)$.

Hypoxia, ischemia/reperfusion and nephrotoxic agents can have rapid and sustained effects on cellular ATP levels in vivo or in vitro. $(241,281,355,401,524)$. Using both standard biochemical approaches and ${ }^{31} \mathrm{P}$ NMR, Stromski et al., demonstrated a rapid reduction of 
renal content of ATP within minutes of the onset of ischemia (524). The restoration of ATP is biphasic upon reperfusion with a rapid initial phase and a more prolonged phase up to 2 hours. If ischemia is short (i.e., $\sim 15 \mathrm{~min}$ or less), ATP levels quickly return to control values. However if the ischemic time in extended (e.g., > $30 \mathrm{~min}$ ), tissue ATP recovers only partially within 2 hours. There is a significant negative correlation of the $2 \mathrm{hr}$ ATP level and the resultant degree of renal function at 24 hours (524). Similarly, in a model of hypotension induced AKI, the early recovery of ATP was impaired in animals that subsequently developed kidney damage, while animals that manifested rapid restoration of ATP were sparred from significant injury (454). These observations suggest a tight link between tubular energetics and a transition from pre-renal azotemia to intrinsic renal failure.

Both ATP depletion and associated cell damage are readily reversible provided substrate can be replenished and the machinery for ATP generation has not been dramatically compromised. In a series of studies from Siegel's group, $\mathrm{ATP}-\mathrm{MgCl}_{2}$ administration to post-ischemic rats accelerated the recovery of tissue ATP that was associated with the preservation of GFR, renal blood flow, and several indices of cellular injury (162-164, 501, 503). Supplementation with inosine, an ATP substrate precursor, and guanine analogs to maintain GTP levels in both in vivo and in vitro models of injury have been shown to have a modest degree of renal protection $(146,258)$.

III.1.2. Loss of structure with impaired energy status-As described above, one of the earliest morphological features in acute injury is the rapid loss of the renal brush border, which occurs within 15 min of reperfusion and is due to the breakdown of cytoskeletal elements (573). The cytoskeletal response is attributed the sensitivity of actin polymerization on cellular ATP levels. The reduction in cellular ATP results in a loss of (filamentous) f-actin from apical microvilli, terminal web junctional complexes and stress fibers $(253,377,378)$. This activity also disrupts cytoskeletal association of protein complexes mediating cell-cell interaction as well as cell substrate interactions, which contribute to the loss of cell polarity, the mixing apical and basolateral transporters and loss of tight junctions (377).

The dynamic processes of actin polymerization/depolymerization become dysregulated when ATP levels are reduced. There is a counter intuitive increase total cellular (filamentous) f-actin formation and a decrease in monomeric g-actin content that occurs within 5 minutes of ATP depletion resulting in the formation of punctuate $\mathrm{f}$-actin structures in the perinuclear region (382). Under physiological conditions, ATP-G-actin monomers, via their association with a class of cytosolic proteins known as thymosins, are prevented from polymerizing with established actin filaments or other G-actin monomers. In the setting of ATP depletion, thymosin dissociates from G-actin monomers, predisposing unregulated factin formation (18).

Ashworth and colleagues suggested that the disruption of the actin network is mediated cofilin/actin depolymerizing factor (ADF). ADF is dephosphorylated and localizes from a diffuse cytosolic location to the apical microvilli and submembrane spaces within minutes of renal ischemia (490) (Figure 5). ADF has been localized in brush border membrane vesicles sloughed into the tubular lumen (15) and can be found in microvessicles in the urine samples from patients with AKI (376). The dependence of ADF activity on the stability of the actin network was demonstrated by studies in which a constitutively active form of ADF disrupted cortical f-actin structures in kidney cells, while cells containing only an inactive mutant of ADF/cofilin did not manifest breakdown of the cortical f-actin network in response to ATP depletion (15). 
$\mathrm{ADF} /$ cofillin activity is regulated by the Rho GTPase, RhoA. RhoA is a member member of the Ras superfamily of p21 G-proteins that are regulated by guanine nucleotide binding. When GTP is bound to RhoA it is active, but intrinsic GTPase activity results in an inactive GDP-bound form (52). GTP levels fall in parallel to ATP in the setting of ischemic injury or in renal cells in response antimycin A and this decreases abundance of the GTP-bound form of RhoA (186). Constitutively active RhoA mutant proteins (RhoV14) preserves cortical factin networks and cytoskeletal ezrin activity. Inactivation of Rho with the bacterial toxin C3 mimics ischemia, results in stress fiber reduction and an impairs recovery of the cortical actin network with the re-establishment of ATP levels (186).

Alterations in cytoskeletal structure lead to a loss of epithelial polarity and barrier function. Cell polarity is established in part by the function of tight junctions and adherens junctions, both of which have a similar architectural network consisting of transmembrane bridging proteins and cytoplasmic proteins linking to the actin cortical network (290). Not surprisingly, ATP depletion alters the cellular distribution of TJ proteins occludin ZO-1, ZO-2 and cingulin $(21,80,176,558)$. In cultured cells and in response to ischemia, there is a degradation of E-cadherin and a disruption of its interaction with catenin both in cultured cells and in ischemic kidney of rat (348). The loss of these junctional structures allows transmembrane proteins with restricted domains to move freely to other membrane spaces. The Na-K ATPase moves from a Triton X-100 insoluble fraction to a Triton X soluble fraction following ischemia in vivo or ATP depletion in vitro. This event is indicative of this protein's release from a restricted basolateral localization, which contributes to the loss of vectorial transport of $\mathrm{Na}$ in the proximal tubule $(379,380)$. The re-localization of $\mathrm{Na}-\mathrm{K}$ ATPase can occur with repletion of ATP by a recycling of Na-K-ATPase subunits (569).

Alterations in cell-cell junctions result in decreased in transepithelial electrical resistance suggesting an increased leakiness in the paracellular pathway (348). This reduced transepithelial resistance is associated with increased backleak of filtrate and was suggested to occur in vivo by studies in which $3[\mathrm{H}]$ - inulin microinjected into the lumen of postischemic proximal tubules was subsequently recovered in the urine formed by the contralateral kidney (133). Similar results have been described in human transplant patients. In a study by Kwon et al., the fractional excretion of graded molecular weight dextrans was increased in patients with sustained AKI, indicative of backleak of inulin during the clearance procedure (284).

Proximal tubule cells require attachment to the basement membrane for proper integrity and function. Cell-matrix adhesions are mediated by the integrin family of cell adhesion receptors, multi-subunit proteins that span plasma membrane and mediate substrate attachment at focal adhesions (FAs)(187, 649). $\beta 1$-Integrin is the most prominent integrin mediating PTC-substrate adhesion and is lost from the basolateral membrane region during the ischemic period $(173,649)$. Goligorsky et al., have proposed that the loss of the basolateral expression of integrin receptors is responsible for the exfoliation of viable proximal epithelial cells and that the redistribution of integrin receptors from the basolateral to the apical surface of epithelial cells facilitates self-adhesion and clumping of detached cells in situ (173). These two processes culminate in tubular obstruction and may severely increase tubular pressures and impair fluid flow. This hypothesis was supported by a series of studies in which cyclized RGD peptides inhibiting $\beta 1$ integrin binding mitigated the formation of obstructive luminal cells, decreased intratubular pressures, and improvement in serum creatinine following renal ischemia reperfusion $(400,459)$.

\section{III.1.3. Impaired renal energetics and the role of reactive oxygen species-}

Replenishment of energetic substrates can resolve the early alterations in cell morphology, but irreversible cell damage may ensue if not adequately restored. Impaired energetics 
represents a potential link toward intrinsic tissue damage and results from various interrelated features of metabolism.

1. Substrate availability: Prolonged ischemia/hypoxia may impair the cellular substrate pool for the re-synthesis of ATP, as originally proposed by Fernando et al. (146). When ATP is degraded, ADP and AMP are formed. The activity of 5, nucleotidase with AMP forms adenosine, inosine and hypoxanthine. Although the nucleotides (ATP ADP AMP and IMP) have low permeability to the cell membrane, the nucleosides adenosine and inosine, and hypxanthine are permeable and can readily diffuse out of the cells (599). Furthermore, hypoxanthine can be converted by xanthine oxidase to uric acid. Thus, as a result of either diffusion out of the cells or metabolism, there is a decrease in the substrate pool for ATP synthesis (599).

2. Renal hemodynamics: The renal outer medulla sits on the "brink of hypoxia" with $\mathrm{pO}_{2}$ values between $10-20 \mathrm{~mm} \mathrm{Hg}$ (compared to $50-60 \mathrm{~mm} \mathrm{Hg}$ in the cortex). With regard to energetics, impaired renal medullary blood flow can further exacerbate hypoxia in the early phases of ischemia reperfusion in rodents (70). In humans, hypoxia is worsened in delayed vs. immediate function grafts following transplant as demonstrated using blood oxygen level dependent (BOLD) MRI (126). The sustained hypoxia may inhibit the resynthesis of ATP accounting for a greater degree of injury in this region.

3. Impaired mitochondrial activity plays a significant role in the injury process and has implications regarding energetics, activation of cell death pathways and the genesis of reactive oxygen species. The effect of mitochondrial function in the setting of injury has been a central focus of not only acute kidney injury, but also injury in myocardium, brain, liver and other organs susceptible ischemic and toxic insult $(44,299,415)$.

Increased mitochondrial cell swelling is a common morphological feature of early response to hypoxia, ischemia/reperfusion and nephrotoxins, in vitro or in vivo. For example, mitochondrial swelling is evident by electron microscopy within 1-2 hours of ischemia reperfusion injury in both proximal convoluted and proximal straight tubules, and the increased severity of ischemia was associated with the severity of mitochondrial swelling (169-171). Mitochondrial swelling is also observed in response to gentamicin treatment (506) and mercuric chloride. In vitro, mitochondria of isolated proximal tubules swell in response to hypoxia/reoxygenation (491). Human kidneys subjected to anoxia during cold storage manifest mitochondrial swelling, release cytochrome $\mathrm{C}$ and demonstrate bax translocation consistent with the activation of apoptotic pathways (see below; (471)). Thus, early alterations in mitochondrial structure represent an important early event in AKI.

III.1.3.1. Respiration and Mitochondrial Permeability Transition: Impaired mitochondrial respiratory capacity appears tightly linked to altered mitochondrial structure. Studies using isolated mitochondria from kidney or cultured kidney cells report reduced ATP synthesis, and respiratory capacity secondary to ischemia, cisplatin (69), lead administration $(154)$ or genatmicin treatment $(308,506)$. Using both in vivo models or preparations of isolated tubules, state II, state III and 2,4 dinitrophenol uncoupled respiration are reduced within 1-2 hours of hypoxia or administration of $\mathrm{HgCl} 2(126,159,164)$. Mitochondrial functional deficits also develop due to the loss of mitochondrial membrane potential (144) and the impaired ability to utilize complex I substrates (602).

The connection between swelling and impaired energetic function may result from an increase in the permeability of the inner mitochondrial membrane (178). Mitochondrial permeability transition (MPT) refers to the process by which the mitochondria lose the 
ability to maintain the $\mathrm{H}^{+}$ion gradient necessary for the production of ATP via the $\mathrm{F}_{1} \mathrm{~F}_{0^{-}}$ ATPase. Substantial evidence suggests that the permeability is due to the opening of a nonspecific pore with a high conductance of molecules less than $\mathrm{M}_{\mathrm{w}} \sim 1200(105,267)$. The result is the loss of the mitochondrial transmembrane potential $\Delta \psi$ accounting for the deenergization of mitochondria and impaired ATP synthesis. The increased permeability can result in the influx of ions such as $\mathrm{K}+$ down its concentration gradient, which is thought to contribute to the observed swelling $(105,267)$.

MPT is commonly studied by evaluating swelling using changes in light absorbance and this approach has been used to identify factors that activate MPT. Most notably $\mathrm{Ca}^{++}$can activate MPT in isolated mitochondria from liver hepatocytes (300) or in permeabilized renal tubule cells at concentrations as low as $35-45 \mu \mathrm{M}(145)$. MPT can also be stimulated by reactive oxygen species by addition of hydrogen peroxide (538), tert-butyl hydroperoxide (268) or xanthine/xanthine oxidase (344) Other potentially important signals associated with renal injury may participate in altering mitochondrial potential. Phospholipase A2 (PLA2) participates in MPT activation suggesting a role for fatty acid (273). Interestingly, Feldkamp et al, demonstrated that delipidated BSA, which can bind free fatty acids in permeabilized tubules, can protect against MPT in response to hypoxia (145).

Permeability transition is thought to be mediated by the opening of the MPT pore, proposed to comprise the adnenine nucleotide transporter (ANT) and the voltage dependent anion channel (VDAC) normally associated in the outer membrane. An in depth summary of the MPT pore is outside the scope this discussion and the reader is encouraged to see recent review articles on its biochemical properties $(105,267)$. Evidence for a specific MPT pore as a physiologically regulated entity derives in part from the discovery that cyclosporine $\mathrm{A}$ inhibits MPT at low and saturable concentrations (299). Cyclosporine reduces ischemic myocardial and hepatic injury $(11,299)$, and it also reduces MPT in rabbit renal tubules exposed to hypoxia (144). However, cyclosporine is not useful in AKI due to its vasoconstrictor effects which likely contribute to worsened kidney injury following ischemia (49). Because cyclosporine binds cyclophilin D (CypD), it has been hypothesized that cyclophilin D, may be a part of and/or regulate the MPT pore complex (397). Neurons from cyclophilin D knockout mice, have a reduced level of MPT in response to oxidant stress when compared to neurons from wild type animals (478). The importance of cyclophilin D in MPT in kidney injury was recently highlighted by Devalaraja-Narashimha et al., who reported that cyclophilin D null mice were functionally and morphologically protected from renal ischemia reperfusion injury (122). In addition to cyclosporine A, MPT activity can also be inhibited by $\mathrm{Mg}++$, low $\mathrm{pH}$ and opening of mitochondrial $\mathrm{K}$ channels by valinomycin (297).

Despite tremendous interest in MPT, other mechanisms for mitochondrial swelling may play a role in tubular cell injury. For example, cadmium may cause swelling independent of the classic MPT; in renal tubule cells cadmium induced mitochondrial swelling that was not blocked by cyclosporine A, but rather was blocked by $\mathrm{AgNO} 3$ and the mitochondrial calcium uniporter (MCU) inhibitor Ruthenium Red (294).

The potential to study MPT within intact cells and tissues has been enhanced by advances in fluorescence imaging and the development functional mitochondrial probes. For example, in cultured rat hepatocytes, the low molecular weight fluorescent compound calcein distributes from cytosol to mitochondria in response to hypoxia, suggesting increased mitochondrial permeability to low-molecular weight substances. Simultaneously, tetramethylrhodamine methylester (TMRM), a red-fluorescing cationic dye that accumulates electrophoretically into mitochondria is depressed in hepatocytes in response to hypoxia (440). Similar results have been shown in kidney tissue slices in vitro. Hall et al., using 2 photon microscopy, 
demonstrated that proximal tubule mitochondrial transmembrane potential $\Delta \psi$ was severely disrupted in slices subjected to chemical anoxia (Figure 6)(185, 601). Theoretically, these approaches could be applied to intact blood perfused whole organs (474). Other potentially important markers of mitochondrial membrane potential including JC-1 and safronin-O have been utilized in models of culture cell injury and isolated tubules $(145,602,618)$, but have not yet been optimized in intact organs with live imaging techniques.

Brooks et al have reported mitochondrial fragmentation may contribute to outer membrane permeabilization. Mitochondrial size and structural integrity is mediating by processes of fission and fusion. Mitochondrial fission is more pronounced following ATP depletion in cultured proximal tubule cells and this change is thought to contribute to outer membrane permeabilizataion and consequent apoptosis (76). Mitochondrial fission is regulated in part by Dynamin related protein-1 (Drp1), which translocates to the mitochondria during early tubular injury. Inhibition of Drp-1 via gene knockdown or dominant negative approaches protected against mitochondrial fission and cell death. A pharmacological inhibitor of Drp-1, known as Mdivi-1, was able to block cell death in vitro as well as in response to ischemic or nephrotoxic damage in vivo (76).

III.1.3.2. Reactive oxygen species: Reactive oxygen species formation in the kidney likely plays a significant role in the genesis acute injury. Evidence has implicated ROS activity as being important in AKI in models of ischemia/reperfusion $(106,127,392)$, cisplatin (23), mercury (417), gentamicin $(338,351)$, endotoxin (620) and glycerol (631). ROS may also influence hemodynamics and inflammation (See Section II). In this section, we will focus on the generation of ROS as a cellular response to injury, and the influence of cellular defense mechanisms in response to injury, occurring secondary to alterations in tubular energetics and mitochondrial function.

The generation of ROS secondary to disturbances in intermediary metabolites in AKI has been the subject of several reviews $(392,521,599)$. Reactive oxygen species of significant interest to renal injury include superoxide anion, hydrogen peroxide, hydroxyl radical as well as nitrositive reactive products nitric oxide and peroxynitrite.

Superoxide is generated as oxygen accepts a single electron. This is a highly reactive shortlived radical form that is dismutated to hydrogen peroxide in the presence of superoxide dismutase (SOD) $(392,521,599)$. Studies in the rat post-ischemic kidney suggested that type $\mathrm{D}$ xanthine oxidase (xanthine dehydrogenase) is converted to type $\mathrm{O}$ xanthine oxidase, which uses oxygen, rather than NAD as an electron acceptor (392). During ischemia, hypoxanthine and xanthine are produced as biproducts of ATP metabolism. A role for xanthine oxidase activity in AKI has been suggested by studies in which allopurinol reduced oxidant activity and mitigated injury secondary to I/R (456). However, several other studies have found inhibition of xanthine oxidase to have minimal effects in models of AKI (128, 634). Moreover, the activity of this enzyme is relatively low in human kidney vs rodent kidney, suggesting that its impact in human AKI is unlikely(392).

The altered fidelity of the mitochondrial electron transport chain represents another potential source of ROS contributing to AKI. Significant levels of reactive oxygen species have been measured in isolated mitochondria from injured rat kidney, despite an overall decrease in oxygen consumption (175). This may be due to impaired electron flow at succinatecytochrome c stage and ROS production at the NADH dehydrogenase and ubisemiquinone sites (175). Blockade of the mitochondrial electron transport chain increases superoxide detectable by dihydroethium fluorescence in kidney slices (185). Infiltrating leukocytes with high levels of NADPH oxidase activity represent a further source of superoxide in AKI (392). 
Sirt1, a NAD-dependent protein deacetylase, is reported to regulate intracellular metabolism and attenuate reactive oxidative species generation from mitochondrial activity. When stimulated pharmacologically, Sirt 1 can protect renal tubules against oxidant induced injury (159). When overexpressed in proximal tubules of transgenic mice, Sirt1 transgenic mice are protected from cisplatin induced injury and show reduced ROS stress and an increase in anti-oxidant enzyme expressions such as catalase (193).

Sirt-1 is also an activator of the peroxisome proliferator activated receptor $\gamma$ coactivator $1 a$ (PGC-1a) a master regulator of mitochondrial biogenesis and adaptative response to hypoxia (499). The up-regulation of this pathway via adenoviral constructs promotes energetic recovery of tubules following oxidant or toxic injury (453). Recently, isoflavones have been shown to upregulate the activity of this pathway to promote mitochondrial biogenesis via effects on Sirt-1(452).

Liberated iron may contribute to oxidant stress via the catalysis of the Fenton reaction (392, $521,599)$. Iron, typically found in storage sites in plasma proteins and heme groups, may be liberated in response to injury and become available for interaction with existing hydrogen peroxide to generate the more insidious hydroxyl radical. Studies using iron chelators such as deferoxamin reduce oxidant associated lipid peroxidation and improved function following ischemia reperfusion or cisplatin $(27,216,419,420)$.

In order to neutralize the effects of reactive oxygen species, renal cells contain a substantial antioxidant defense mechanism. The kidney contains 3 forms of superoxide dismutase, (SOD1/ CuZn SOD, SOD2 Mn SOD, and extracellular SOD/ecSOD/SOD3), catalase and glutathione $S$ transferase. These defense systems are impaired during injury and their activity may modulate the severity of AKI. Cu/Zn SOD, catalase and glutathione levels and glutathione $S$ transferase expressions are reduced in most models of renal injury, although there may be a modest increase in Mn SOD following ischemia reperfusion injury $(127,221$, $289,485,508,625)$. The further impairment of these intrinsic defense mechanism results in worsened outcome in AKI; for example, SOD1 null mice develop more severe AKI to ischemia reperfusion than corresponding wild type mice (611), and chronic exposure of rats to a hypoxic environment reduces renal SOD expression and results in increased sensitivity to AKI (517). It is interesting to note that a recent report demonstrated a significant association between serum catalase activity and the severity of AKI in patients with catalase allele $\mathrm{C}$ to $\mathrm{T}$ at position -262 (431), suggesting that the intrinsic antioxidant defense pathways of individuals may vary and influence the course of AKI.

Enhancement of intrinsic anti-oxidant mechanisms has protective benefits in models of AKI. Administration of superoxide dismutase decreases severity of AKI in rats $(43,421,482$, 556) and dogs (278), while an increase CuZn SOD gene expression by adenoviral gene transfer protects renal cell death in vitro and in vivo in response to ischemia reperfusion $(314,621)$. Pharmacological agents such as tempol, which possess SOD mimetic activity, attenuate the degree of renal injury in endotoxemia and I/R models $(87,289)$. Similarly, MnTMPyP, possessing both SOD and catalase activity and protects against renal ischemia reperfusion (386). Supplementation with glutathione $(1,184,418,600)$ or the glutothione precursor $\mathrm{N}$-acetylcysteine (NAC) prevents the drop in renal glutathione content in response to injury, attenuates oxidant stress and the severity of renal injury in a variety of AKI models $(98,125,398)$. However, these approaches tend to have small modulatory roles on the extent of injury and may have limited therapeutic potential. In clinical trials, NAC did not influence AKI incidence or length of stay following cardiac surgery $(2,220)$. 


\section{III.2. Cell death in the setting of AKI}

An examination of cellular injury in AKI inevitably results in a discussion regarding the mode of cell death that occurs in the kidney and the potential distinction between necrotic death and apoptotic (also referred to as programmed cell death, PCD) forms of cell death. The reader is referred to several excellent reviews dedicated to this subject $(415,475)$. The terms apoptosis and necrosis arise from classical pathological terms based on well-defined morphological distinctions. Both apoptosis and necrosis utilize distinct and common biochemical processes; as this represents an area in which potential therapeutic targets may emerge, the study of these processes is an area of intense investigation.

III.2.1. Apoptotic and necrotic cell death and their presence in AKI-The term apoptosis (from greek: apo-from, ptosis falling) was originally described by Kerr in 1972, emphasizing its role in normal cell turnover and distinguishing it from necrotic cell death following traumatic injury (263). In its classical form, apoptosis is a highly ordered process mediated by pre-existing enzyme cascades and requiring energy in the form of ATP.

The earliest morphological features of apoptosis are defined by cytoplasmic and nuclear shrinking, chromatin fragmentation and the breakdown of the cell into multiple spherical bodies (i.e., apoptotic bodies) that retain membrane integrity (415). A hallmark feature of apoptosis is the retention of membrane integrity, which prevents spilling of cellular/ lysosomal contents into the interstitium, preventing an inflammatory reaction and limiting the extent of surrounding tissue damage (415). Although membrane components remain intact, phosphatidyl serine residues that are localized in the inner membrane are exposed on the outside of plasma membrane prior to cellular fragmentation where they are involved in targeting cells of the resultant apoptotic bodies for phagocytic engulfment by macrophages or neighboring parenchymal cells. Therefore, when apoptosis occurs on a limited basis during normal cell turnover and in response to modest injury, there is little effect on the surrounding tissue (415).

In contrast, the morphology of necrosis is distinctly different and is characterized by swelling and degeneration of the entire cytoplasm. The chromatin is not packed into discrete membrane bound particles, but forms irregularly shaped clumps. There is dilation and fragmentation of the cisterns of the rough endoplasmic reticulum and polyribosomes are dissociated and dispersed throughout the cytoplasm. The plasma membrane does not remain intact and there is blebbing of the plasma membrane with shedding of the cytoplasmic contents, likely exacerbating inflammatory reactions and expanding the degree of local injury (Figure 7) (415).

Within the setting of acute kidney injury, both apoptosis and necrosis may occur simultaneously. Using protocol biopsies following transplant or post mortem histological analysis of patients with AKI, demonstrates the presence of both necrotic and apoptotic cells (554). In rat ischemia reperfusion injury, Schumer et al, demonstrated that a dose dependency of apoptosis and necrosis in response to ischemia reperfusion injury in rats such that low levels of ischemia ( $15 \mathrm{~min})$ manifested only apoptosis and no necrosis. Increasing levels of ischemia were associated increasing appearance of apoptosis as well as necrosis (489).

Although apoptotic and necrotic pathways will be discussed below, we suggest these distinctions should not be viewed sharply. Rather, within the context of renal injury, we suggest that apoptosis and necrosis are not functionally opposed forms of cell death, but rather represent manifestions of death within a spectrum of injury, as put forth by Lieberthal et al., (319, 321)(Figure 8). Because apoptosis requires ATP, and ATP prevents the onset of necrotic cell death, the form of death is dependent on the degree to which ATP is depleted. 
Using in vitro models of proximal tubule cells subjected to chemical anoxia, when ATP levels fall below $15 \%$ of control values, necrosis ensues. In contrast, when ATP levels are reduced to between $25-50 \%$ proximal tubule cells undergo predominantly apoptotic cell death (321). Dose dependency has been observed in response to cisplatin (286), cyclosporine (199), oxidant injury by $\mathrm{H}_{2} \mathrm{O}_{2}$ and other ROS generating models of injury (564).

The blurred distinctions between apoptosis and necrosis has led Lemasters to propose the term necrapoptosis, to describe the observations demonstrating that both forms of cell death can be observed in tissues subjected to ischemic or toxic insults (298). The term necrapoptosis suggests that both apoptosis and necrosis can be triggered by a common mechanism, but culminates in either cell lysis (necrosis) or programmed self destruction (apoptosis) depending on the nature of other modifying factors (298). The hypothesis is predicated on the idea that the biochemical pathways leading to classical necrosis and apoptosis overlap and interact. Primary among these pathways is the central role that mitochondrial permeability transition plays in triggering the activation of classic apoptotic stimuli and regulating cellular ATP levels (298).

Viewed in this way, the hypothesis put forth by Leiberthal et al., is similar to the idea of necrapoptosis proposed by Lemasters. During the course of apoptotic cell death, if injury is severe and ATP depletion occurs, a secondary necrotic death will intervene. If ATP levels are maintained, cell death occurs primarily by apoptosis. The dose dependency of ATP on the type of cell death may account for the in vivo observation that necrosis is more prominent in the outer medulla where $\mathrm{pO}_{2}$ reductions are exacerbated by vasoconstriction or congestion, thus prolonging or exacerbating the degree of ATP depletion in this region as compared to a relatively better-perfused cortical zone.

In AKI, the preponderance of apoptotic vs. necrotic cell death may differ substantially depending on the experimental parameters such as the agent, dose and species being investigated. It is widely recognized that differences in sensitivity to injury are prevalent across species. Rats and mice both show overt necrosis in response to I/R injury. Mice typically manifest significant injury with shorter ischemic times in the range of 25 minutes (609) with some reports of significant necrosis resulting from an injury in as little as 17 minutes (266). Studies in rats utilize longer ischemic times (60-60) minutes of ischemia (554) although some studies have demonstrated necrosis in rats with as little as 25 minutes of ischemia (573). As mentioned earlier, although evidence of tubular damage is seen in human kidneys, necrosis is patchy following ischemia suggesting an overemphasis of cell death in rodent models (323). This may relate to the size of the organism and the degree to which juxtamedullary blood flow is affected in larger kidneys. In dogs, juxtaglomerular flow is not dramatically reduced following release following clamps over 120 minutes. There is little evidence of overt necrosis despite the persistent evidence of sublethal injury (323, 458). Similarly, pig models show modest necrosis with up to 3 hours of ischemia $(26,462)$.

Although mercuric chloride toxicity has long been reported to be associated with the rapid development of renal epithelial necrosis (566), models using toxins to induce AKI are usually executed to develop more slowly and show a more limited necrotic response. This may be related to the doses and timing used for each study. Cisplatin injury shows a peak onset of injury as late as 3 days following the administration of the agent and is characterized by a mix of apoptotic and necrotic death, although apoptotic cell death is readily identifiable in this model is early as 2 days post-injection $(362,450)$. Typically, many nephrotoxic agents (e.g., cisplatin, gentamicin, mercury, chromium), will manifest evidence of necrosis by 3 days, while manifesting evidence of sublethal damage at earlier time point $(211,442,644)$. All of these agents are reported to induce apoptotic pathways in 
vivo and in vitro $(211,281,388,442,450,466,493)$ suggesting that much of the necrosis observed is secondary.

III.2.2. Biochemical pathways mediating necrosis-Necrotic cell death is historically viewed as a passive process secondary to the breakdown of cellular energetics. Reductions in cellular ATP levels result in reduce Na-K ATPase activity and net Na influx associated with swelling of the cytosolic compartment $(62,353)$. Although the site of passive sodium entry in ATP depletion remains elusive (415), the associated cell swelling is thought to be antecedent to necrotic death. Zager et al., demonstrated that mannitol protected against cell swelling in ischemia reperfusion, but did protect against oxidant stress, mitochondrial swelling or energetics (630). Similar protection from cellular swelling was also observed in rhabdomyolsis model induced by glycerol, however mannitol actually impaired energetic recovery in this model (633).

Histological evidence of necrosis is typically, but not exclusively, observed in proximal tubules, while sparse in the distal tubule and TAL. Among the possible explanations for this difference is the relatively high hydraulic conductivity of proximal tubule membranes that contribute to swelling secondary to $\mathrm{Na}$ influx (61). Aquaporin-1 is the primary water channel in the proximal tubule that contributes to water reabsorption and therefore, it may contribute to cell swelling. In contrast, the plasma membranes of the TAL and DT are largely impermeable to water. The idea that aquaporins contribute to swelling associated with necrosis is supported by studies showing that aquaporin 4 null mice are protected from edema in various brain injury models (e.g., glutamate, focal ischemia) (349). Although aquaporins have been investigated with regard to diuretic activity in acute kidney injury, no studies have examined their potential contribution toward osmotic cell swelling in AKI.

The potential role of $\mathrm{Ca}^{++}$ion in mediating renal damage in acute injury has been a major area of research for over 30 years. A role for $\mathrm{Ca}$ in the contribution to renal injury is suggested due to the protective effects of $\mathrm{Ca}^{++}$channel blockers $(486,487)$. Verapamil, a slow $\mathrm{Ca}^{++}$channel antagonist, conveys protection in a model of norepinephrine induced AKI $(486,487)$. Both verapamil and diltiazem have been used to block AKI associated with transplant and both provided significant protective effects to renal allografts (116).

However, verapamil does not directly protect renal tubule cells in suspension, suggesting that $\mathrm{Ca}^{++}$channel antagonism is directed at renal vascular impairment rather than cell injury pathways (60).

Nevertheless, injured or dying cells accumulate cytosolic $\mathrm{Ca}^{++}$thought to derive from both intracellular and extracellular sources (60). There is a $\sim 10,000$ fold gradient of $\mathrm{Ca}^{++}$ concentration ( $1 \mathrm{mM}$ extracellular vs $100 \mathrm{~nm}$ intracellular) that is normally maintained by active energy requiring extrusion mechanisms. The sarcolemmal Ca-ATPase actively pumps $\mathrm{Ca}^{++}$into the ER compartment, representing a high capacity reserve depot for excess $\mathrm{Ca}^{++}$. This system is compromised when ATP levels are reduced (60). Proximal tubular free ionized cytosolic $\mathrm{Ca}^{++}$rises from values of 170 to $390 \mathrm{mM}$ during a 5 min hypoxic injury $(486,487)$. The increased $\mathrm{Ca}^{++}$is readily reversible with brief hypoxic insults but with prolonged hypoxia or ischemia, $\mathrm{Ca}^{++}$remains elevated and may participate in injurious processes (vide infra) Reductions in extracellular $\mathrm{Ca}$ or intracellular sequestration of intracellular $\mathrm{Ca}^{++}$conveys cytoprotection to renal cells in suspension or in culture (607).

The $\mathrm{Na} / \mathrm{Ca}$ exchanger typically couples $\mathrm{Na}$-entry with $\mathrm{Ca}^{++}$release; under conditions of elevated intracellular $\mathrm{Na}$, this exchanger may work in reverse mode and promote the build up of intracellular $\mathrm{Ca}^{++}(60,415)$. Mice heterozygous for the NCX1 gene express approximately $50 \%$ of $\mathrm{Na} / \mathrm{Ca}$ exchanger protein in proximal tubules. Cultured proximal tubules from these mice demonstrate reduced $\mathrm{Ca}^{++}$influx to hypoxia, while kidney function 
and cellular damage in vivo are minimized following ischemia reperfusion injury (612). Moreover, drugs which prevent the $\mathrm{Na} / \mathrm{Ca}$ exchanger from operating in reverse mode attenuate kidney injury in response to ischemia reperfusion injury $(406,612)$.

$\mathrm{Ca}^{++}$regulates a number of processes that influence cell injury. As described above, increased cytosolic $\mathrm{Ca}^{++}$may influence the process of $\mathrm{MPT}$. $\mathrm{Ca}^{++}$may also participate in activation of $\mathrm{Ca}^{++}$dependent proteases, phospholipases and endonucleases; processes described further in the following sections.

\section{III.2.3. Biochemical pathways involved in apoptosis and their involvement in}

AKI-Apoptosis is regulated by several interrelated biochemical pathways targeted toward the programmed self-destruction of the cell in an orderly fashion (Summarized in Figure 9). A hallmark feature of apoptosis is degradation of DNA by specific endonuleases. Endonuclease activity at accessible areas between nucleosomal regions results in the characteristic "DNA ladder", while necrotic cells demonstrate a less orderly smearing pattern. Nuclease activation represents a point of no return in the injury process. Basnakian et al, identified abundant expression of the gene encoding DNAseI in rat kidney and demonstrated that its activity was increased in response to ischemia reperfusion injury and cisplatin $(39,41)$. Specific inhibition of DNAse I with antisense oligonucleotides protected tubular epithelial cells against hypoxia reoxygenation in culture (41).

There is also evidence for other endonucleases activated in response to injury. For example, Endonuclease G (Endo G) is a small $27 \mathrm{kDa}$ protein residing in the inner membrane space of mitochondria and can traverse to the nucleus under apoptotic conditions (313). In rat kidney tubular epithelial cells, Endo G was transported to the nucleus in response to hypoxia reoxygenation. The increased mitochondrial permeability was shown to be dependent the production of ceramide, since the process was inhibited by fumosin B1, an inhibitor of spingomylinase (40). Both DNAse I and Endo G may play complimentary and synergistic roles in endonuclease activity in models of AKI; using DNAse I null mice, Endo G was found in nuclei of proximal tubule cells following cisplatin injury while inhibition of Endo $\mathrm{G}$ in vitro protected against DNA strand breaks in response to cisplatin (622). In addition, another endonuclease, termed apoptosis inducing factor (AIF), may be activated in response to cisplatin injury (333).

III.2.3.1. Initiation and downstream signaling of apoptosis in kidney injury: Caspases, or cysteine-aspartic proteases, are a family of enzymes which are thought to play an essential role as primary initiators of cell death. Caspases are constitutively expressed in a latent form and activated in response to a variety of potentially apoptotic stimuli. They are typically described as comprising a canonical pathway in which initiator caspases (caspases-2, -8, -9, 10) are sensitive to an initiating signal, self activate, and then act upon subsequent "effector" caspsases (caspase-3, -6,-7). Effector caspases are in turn responsible for activating pathways associated with the orderly destruction of the cell (e.g, DNAse and protease degradation). Historically, apoptotic pathways are considered to be activated from signals associated with intrinsic cell stress, or from factors extrinsic to the cell (Summarized and reviewed in $(413,415,563))$. Therefore, cell death may occur via common mechanisms regardless of the stimuli, as initiator caspases associated with extrinsic and intrinsic pathways both converge on the common effector caspases. Both caspase 3 and caspase 6 are expressed in kidney and activated in response to injurious stimuli $(143,252,366,493)$ and caspase 6 , localizes to the nucleus following ischemia reperfusion injury (507). The importance of both intrinic and extrinsic pathways in multiple models of AKI is generally well-accepted based numerous studies, a portion of which are described below. 
III.2.3.2. Extrinsic pathway: The extrinsic pathway is one typically associated with activation of a subset of TNF receptor family cell surface "death" receptors which includes Fas/CD95, TNFR1, and death receptor-3 (415). Engagement of death receptors by their ligand invokes receptor trimerization and complex formation within the cytoplasmic bounds of the receptor with molecules such as FADD/MORT, TRADD and RAIDD, which contain death effector domains (DED). Caspase-8 represents the key initiator caspase of the extrinsic pathway and it is presumed that the association of procaspase- 8 with these DED permits its autoproteolytic activation to caspase- 8 and subsequently caspase- 3 .

Tubular epithelial cells undergo apoptosis in response to members of the TNF ligand superfamily, including TNF-a, FasL (CD95) and TRAIL (475). Administration in vivo of CD95 (Fas) results in endothelial apoptosis, which can be blocked by the inhibitor to caspase-8 or with the z-IETD peptide that selectively inhibits caspase-8 (232). These pathways are particularly relevant in models of sepsis. Cunningham demonstrated that TNFreceptor null mice were resistant to the injurious effects of endotoxin on renal function and apoptotic death (107). Other studies have shown that peptide based inhibitors of caspase 8 as well as caspase 3 attenuated endotoxemic injury (179). In a cecal ligation model of sepsis, the administration of siRNA to block either caspase- 8 or Fas improved outcome and organ apoptosis, although the kidney injury was not a target in that study (603).

The activation of the extrinsic pathway is not exclusive to sepsis models of AKI; Fas mRNA was increased, and immunoreactive Fas protein localized to proximal tubule cells, within hours of renal ischemia reperfusion injury (399). Dendritic cells may be the source of TNFa following renal ischemia reperfusion that could activate this pathway (129). Inhibition of caspase 3 and 8 with siRNA significantly attenuated renal ischemia reperfusion injury in mice, however, since both caspase 3 and 8 were inhibited in this study, it is difficult to distinguish the effects from the intrinsic pathway, which is also active in these settings (640).

The extrinsic pathway may be activated secondary to other inflammatory or modulatory factors. In cultured renal tubular cells, pro-inflammatory cytokines IFN- $\gamma$ and TNFa increase NOS-2 activity and lead to the formation of active caspase- 8 and apoptotic cell death. This effect was blocked not only by L-NMMA, but also several strategies to inhibit caspase 8 such as the caspase- 8 inhibitor z-IETD-fmk, the overexpression of the endogenous caspase-8 inhibitor cFLIP, and silencing of caspase-8 (134). The pro-inflammatory cytokine IL-2 also causes apoptosis in proximal tubule cells, and this effect is reduced in cells from Fas-/- mice and by c-FLIP (135). Natural Killer T cells, (NKT) may also modulate the extrinsic pathway in ischemia/reperfusion injury. The NKT receptor Rae-1 is expressed on the surface of tubular epithelial cells following IR injury; NKT cell interaction can cause cell death in a FasL dependent fashion (641).

III.2.3.3. The intrinsic pathway: The intrinsic pathway is responsive to cell stress and is activated by oxidative stress/reactive oxygen species, radiation, ceramide, and growth factor deprivation. The loss of normal morphology or contact with substratum may also induce apoptosis. It is interesting that the stabilization of the actin cytoskeleton reduces apoptotic death in early IR injury (166).

Features typically associated with the intrinsic pathway are the release of mitochondrial apoptogenic molecules, particularly cytochrome $\mathrm{c}$, the activation of procaspase- 9 , and the interaction of bcl-2 family members. The release of cytochrome $\mathrm{c}$ into the cytosol from the inner memrane space of the mitochondria is sufficient to induce apoptosis in some cell types $(413,415,563)$. Cytochrome c binds and oligomerizes with the adaptor protein apaf-1, 
which in turn recruits and pro-caspase -9 leading to the formation of the apoptosome, and the autocatalytic activation caspase- $9(413,415,563)$.

Activated caspase 9 can proteolytically activate caspase-3. A central role of cytochrome c/ caspase 9 in the intrinsic pathway is demonstrated clearly in classic studies using cell lines derived from cytochrome $\mathrm{C}-/-$ mice, which do not activate caspase- 3 and are resistant to the proapoptotic effects of UV irradiation, serum withdrawal, or staurosporine (vs. wild type derived cells). In contrast, these cells remain sensitive to extrinsic pathway stimulation (310). Similarly, thymocytes derived from Caspase $9-/-$ mice, show reduced caspase-3 activation and cell death induced by etoposide, dexamethasone, and $\gamma$-radiation when compared to wild type cells, and cell death induced by Fas antibody was similar in thymocytes from wild type and caspase $9-/-$ mice (282).

Given the importance of mitochondrial cytochrome c release (and other mitochondrial derived pro-apoptotic factors), the understanding of this step is significantly important to understanding the injury process. The control of mitochondrial permeability is often attributed to members of the bcl-2 family of proteins classified as either pro-apoptotic (bax, bad, bak) or anti apoptotic molecules (Bcl-2, bclXL, Bcl-w, Mcl-1, and A1). Pro-apoptotic Bcl-2 family members (e.g., bax) insert into the outer membrane and increase permeability of the membrane to proteins such as cytochrome c, either by forming channels, interacting with membrane proteins to facility pore activity (i.e., VDAC), or by contributing to membrane depolarization, in which cytochrome $\mathrm{c}$ would be released secondary to swelling (595).

Translocation of bax from cytosolic compartment to the mitochondrial compartment precedes the release of cytochrome c following injury. Saikumar et al., demonstrated that hypoxia leading to ATP depletion resulted in a translocation of bax protein to the mitochondria and release of cytochrome c (469). Caspase inhibitors did not prevent the translocation of bax and the release of cytochrome c; interestingly, caspase inhibition prevented apoptotic cell death but not necrotic cell death, as mitochondria did not recover (469). Others have demonstrated translocation of bax, as well as another pro-apoptotic member, bak, in culture models of ATP depletion (368), cisplatin $(235,388)$, and in vivo within 6 hours of I/R injury (438). Bax mRNA and protein are up-regulated rapidly following I/R injury in the kidney $(36,172)$. Bax null mice were resistant to cytochrome $\mathrm{c}$ release, apoptosis and had preserved renal function and morphology following administration of cisplatin (597).

Two other pro-apoptotic members of the Bcl-2 family have also been identified in kidney injury models; both bad and bid translocate to mitochondria in hypoxic tubule cells and in response to ischemia reperfusion injury similar to bax (596). Data from biopsies suggest that these pathways may operate in human kidneys following transplant (85). Bid activation is mediated by a cleavage event inhibited by caspase 8 or caspase 9 inhibitors, suggesting a potential link between extrinsic and intrinsic pathways. In kidney cells, bid sensitivity to caspase -9 inhibitors suggests that it may amplify the mitochondrial pathway (596). Bid deficiency in transgenic mice was shown to alleviate the extent of renal damage using a model of ischemia reperfusion (598).

The regulation of bax activity involves, in part, interactions with other proteins resulting in both complexity as well as potential avenues for therapeutic intervention. A well-studied area of investigation involves the interaction that occurs with other Bcl-2 family members. Heterodimerization with Bcl-2 can nullify the efficacy of Bax to release cytochrome c (412). Using kidney tubular epithelial cells, Wang et al,, demonstrated that minocycline induced the expression of Bcl-2, accumulating in the mitochondria and interacting with Bax, Bak and 
Bid; specific inhibition of Bcl-2 using an siRNA strategy, reduced the protective effects of minocycline in response to hypoxia, azide, cisplatin and staurosporine (587). Transgenic overexpression of Bcl-2 or BclXL attenuates damage associated with in cardiac ischemia reperfusion and acute liver injury $(92,103,119,373,430)$. Alterations in the activity of Bcl-2 family members may underlie the differential sensitivity of proximal tubular vs. distal tubular injury. Cuttle et al, demonstrated that distal tubule cells in culture express the antiapoptotic protein of $\mathrm{Bcl}-\mathrm{X}_{\mathrm{L}}$, which translocates to the mitochondria in surviving distal nephron cells in response to peroxide treatment, while proximal cells do not express prominent of $\mathrm{Bcl}-\mathrm{X}_{\mathrm{L}}$ or $\mathrm{Bcl}-2$ and die from apoptosis (111).

The reasons why pro-apoptotic Bcl-2 family proteins migrate to the mitochondria remain elusive. It may involve conformational changes affecting protein-protein interactions. JNKa pro death signaling molecule in injured tubule cells, affects bax translocation $(296,559)$. Similarly, inhibition of p53 activity protects in renal ischemia reperfusion injury, in part, by blocking bax translocation (115). A newly identified factor, nucleophosmin is a bax chaperone that may facilitate bax delivery to the mitochondria (264).

III.2.5. Autophagy-Autophagy is a cellular process of bulk degradation of damaged organelles, protein aggregates and macromolecules in the cytoplasm (432). It is a stress response suggested to contribute to cell death. Recently, Periyasamy-Thandavan et al., suggest that this process is renal protective in AKI. In a model of cisplatin induced injury, autophagic vesicles and autophagosomes were identifed in tubular cells prior to apoptotic cell death (433). When autophagy was blocked pharmacologically with 3-methyadenine or bafilomycin, tubular cell apoptosis was enhanced. Similarly, knockout of the pro-autophagic gene, beclin, also enhanced the apoptotic response to cisplatin (433). However, the role of autophagy as contributing toward cell death or cytoprotection in the setting of AKI is controversial. This area is likely to garner increased attention in the near future (130).

\section{IV: Repair and Regeneration}

\section{IV.1. Adaptive nature of kidney and proximal tubules}

As described above the cellular response to injury is heterogeneous, with some cells undergoing necrosis or apoptosis, while others are sublethally injured. The severity and duration of AKI may be related to the proportion of sublethally damaged cells that are able to maintain viability and contribute to a coordinated repair process restoring kidney structure and function. Renal tubular cells are remarkable for their adaptive nature to threatening situations and maintain viability. An examination of gene expression following renal injury reveals groups of genes that are associated with adaptation to injury $(123,529)$. The activation of such pathways represent potential therapeutic targets to lessen the severity of AKI, while the down-regulation of such pathways may exacerbate injury and may represent clinically relevant risk factors for the development or outcome of AKI.

IV.1.1. The renal stress response-Injury to the kidney activates well-defined cytoprotective pathways, referred to as the renal stress response, which can be classified broadly in at least 3 pathways with multiple targets.

IV.1.1.1. Heme Oxygenase (Inducible HO, HSP32) and anti-oxidant genes: The heme oxygenase enzyme system catalyzes the rate-limiting step in heme degradation, producing equimolar quantities of biliverdin, iron, and carbon monoxide (CO). Biliverdin is subsequently converted to bilirubin by biliverdin reductase. Two isoforms of heme oxygenase have been characterized: an inducible enzyme, HO-1, and a constitutive isoform, HO-2 (505). Activity of HO in response to various signals associated with injury is thought to contribute to cytoprotection. In animal models, HO-1 is rapidly induced following 
ischemia/reperfusion (347), glycerol-rhabdomyolysis (391), and nephrotoxic injury (3). HO-1 expression is controlled primarily based on its transcriptional activity and can be influenced by multiple factors including, growth factors, Ang II, inflammatory signals, NO and heavy metals. However, alterations in cellular redox state represents one of the most powerful regulators of HO-1 activity (505). The redox sensitive transcription factor, Nrf2 represents a key regulatory molecule in the activation of antioxidant genes in kidney injury and is a well-described modifier of HO-1 transcription. Mice with a null mutation in the Nrf2 gene demonstrate significantly reduced activation of a number of antioxidant genes such as HO-1 (334). In relation to the mouse promoter, the human HO-1 promoter is much less responsive to factors such as hypoxia, interferon- $\gamma$ and oxidized lipids. However, the human promoter is very responsive to induction by heme (505). Increases in heme may be attributed to increased filtration in the setting of rhabdomyolysis or may be liberated from destabilized proteins in response to ischemia or nephrotoxin induced damage; such increases precede activation of HO-1 expression (505).

The functional importance HO-1 in AKI derives from multiple studies in which HO activity is impaired. For example, administration of tin mesoporphyrin, which blocks HO-1 activity, worsens renal function in response to ischemia/reperfusion (498). Mice with a null mutation in HO-1 show greater loss of kidney function and increased mortality in response to ischemia reperfusion (437), glycerol (391), and LPS (367). Similarly, Nrf2 null mice with reduced HO-1 induction also manifest greater injury to ischemia and cisplatin (334). Conversely, induction of HO-1 expression by infusion of hemoglobin intravenously prior to injury, or viral delivery of HO-1 expressing gene, protects from injury $(392,393)$. Other strategies have been used to increase HO-1 activity such as adenoviral gene transfer (55), and cobalt chloride administration $(96,185)$, which protect against renal injury induced by either warm ischemia reperfusion injury or cold ischemia in transplant models. HO-1 minimizes oxidant stress by virtue of its degradation of heme, a toxic pro-oxidant, as well as its generation of bilrubin, a peroxy radical scavenger that inhibits lipid peroxidation. In addition, $\mathrm{CO}$ is byproduct of the HO-1 activity, providing a potential vasodilatory effect on the renal vasculature and a restoration of blood flow (393). HO-1 expression is present in renal biopsies following transplant and its levels correlate with the degree of post transplant function (301).

IV.1.1.2. Heat shock proteins: Sublethal heat stress transiently increases the ability of a cell to withstand an otherwise subsequent lethal heat challenge. Similar protection is routinely observed in response to diverse stressors such as hypoxia, ischemia, heavy metal exposure and oxidative stress. Interest in understanding this protective response led to identification of a fairly large family of proteins known as heat shock proteins; a multigene family of proteins ranging in size from $10-150 \mathrm{kDa}$ and found in all major cellular compartments (46). Heat stress has similar consequences as hypoxia at the cellular level. For example, both heat stress and hypoxia are settings where damage to the cytoskeleton occurs, where there is mitochondrial swelling, and where there is uncoupling of oxidative phosphorylation. Hence, the term "heat stress protein" has been proposed to reflect this more generic association with cellular stresses (46).

One of the earliest reports suggesting a role for heat shock factors in protection from acute kidney injury was from Emami et al (140); transient minimal ischemia (15 min) resulted in an increase in heat shock protein72 (HSP72), which was associated with protection to subsequent ischemia/reperfusion injury. Whether the protection was actually attributable to HSP72 was not addressed in that study as methodologies to specifically inhibit HSP72 specifically were not available at the time. 
It is now clear that not only HSP 72, but also other HSP family members including HSP10, HSP 25/27, HSP47, HSP70 and HSP90 and a $\beta$-crystallin are substantially induced in diverse models of renal injury including ischemia reperfusion, cisplatin and unilateral ureteral obstruction $(340,484,494,509,570,642)$. The mechanisms of gene induction of the various heat shock proteins is quite heterogeneous, however, many of the inducible forms of HSP (i.e., HSP 72, HSP 27) contain heat shock elements in their promoters, which are activated upon binding of the regulatory protein, heat shock factor (HSF) $(521,570$, 571). Under non-stress conditions HSF is bound to the constitutively-express HSP referred to as heat shock congnates (HSC), often HSP 70. Increased demand for the use of HSC following a stress leads to a dissociation of $\mathrm{HSC} / \mathrm{HSF}$ complex, thus liberating this regulatory molecule and activating transcription (Figure 10) (521, 570, 571).

The protective effect of HSPs is widely attributed to their activity as "molecular chaperones," which are proposed to assist in the assembly or repair of newly synthesized or damaged proteins (65). In vitro, chaperones function to prevent aggregation of other proteins under conditions of stress and to promote restoration of enzymatic activity of denatured protein substrates or enzymes on removal of the stress $(46,65)$. HSPs are found in multiple protein aggregates following the induction of ischemic kidney injury. Hsp72 binds to aggregated cellular proteins, including $\mathrm{Na} / \mathrm{K}-\mathrm{ATPase}$, and is released upon addition of ATP in vitro, a feature typical of its chaperone function (19).

A prominent role of HSPs is associated with the stabilization and recovery of cytoskeletal structure following injury. During recovery following ischemia, the Na, K, ATPase reassociates with the cytoskeleton, as evidence by its reappearance into a Triton X100 insoluble pellet. HSP-70 is also present in the Triton X100 insoluble pellet and correlates with the reappearance of the Na, K, ATPase. When antibodies against HSP-70 are included with the insoluble fractions, the re-association of the Na, K, ATPase with the cytoskeletal fraction in inhibited, while the addition of HSP-70 protein promoted $\mathrm{Na}$, K, ATPase/ cytoskeletal interaction in vitro (50). HSP72 co-precipitates with focal adhesion component paxillin following ATP depletion; the overexpression of HSP 72 hastens the reestablishment of focal adhesions following transient ATP depletion (350).

HSP27 has also been identified in cytoskeletal protein aggregates from injured kidney (567). In cultured proximal tubule cells, HSP27 migrates to the basolateral cell boundaries following ATP depletion Overexpression of either HSP-72 or HSP-27 preserves the alterations in cytoskeletal architecture in response to ATP depletion $(135,176)$. HSP-27 may preserve tight junction formation via an indirect effect on Src-activation (198) .

HSP-chaperone activity may confer cytoprotection by interacting with, and inhibiting the biochemical pathways leading toward cell death. HSP 72 associates with AIF (apoptosis inducing factor) and prevents leakage of AIF from mitochondria, the nuclear accumulation of AIF and subsequent DNA fragmentation in ATP depletion (461). Furthermore, HSP-27 antagonizes conformational changes in Bax and protects mitochondrial injury (197).

HSP family members may also function as cellular antioxidants. In MDCK cells exposed to oxidant stress, HSP-70 prevented the increase in superoxide production by increasing the activity of glutathione peroxidase and glutathione reductase (180). HSP-90 coupled to NOS3 preserves NO synthesis, while when uncoupled from NOS3, leads to the formation of vasoconstrictive superoxide (439). Hypoxia increases HSP-90-NOS3 coupling in coronary endothelial cells. More recently, HSP-90 has also been shown to modulate the vasorelaxation induced by NOS1. Agents such as radicicol inhibit HSP-90/NOS3 interactions and lead to an acute reduction in renal blood flow (451) Therefore, cumulative HSP activity may improve renal blood flow in the regenerating kidney by improving the 
nitric oxide/superoxide ratio. HSPs may also provide protection in the setting of injury by attenuating inflammation. Ischemia/reperfusion-induced NF- $\kappa$ B activation, MCP-1 expression and monocyte infiltration was suppressed by heat preconditioning. Inhibition of HSP-70 by quercetin reversed these effects, while liposomal delivery of HSP 72 into renal proximal tubule cells blocked the activation of NF- $\mathrm{kB}$ in response to in vitro hypoxiareoxygenation $(71,99)$.

Until recently, in vivo studies on HSP function in AKI have been lacking due to the paucity of transgenic models. Transgenic mice overexpressing HSP-70 are protected in a model of cerebral infarction (448), but these mice have not been evaluated in AKI. Recently, Kim et al., demonstrated that the selective overexpression of HSP-27 targeted in proximal tubules conferred resistance to ischemic AKI (270). Genetic factors may influence HSP activity; higher basal levels of HSP-72 and HSP-27 were reported in Brown Norway rats which are resistant to AKI relative to commonly utilized Sprague Dawley rats (30). Other approaches such as preconditioning (described in more detail below) are consistent with the idea that up-regulation of HSP provide protection in AKI. In patients, it has been suggested that genetic polymorphisms in HSPs, specifically, the Hsp72 1267-GG allele may increase the risk of acute kidney injury in low birth weight neonates (572).

IV.1.1.3. Stress activated protein kinases: Cellular stress induced by oxidants and DNAdamaging agents activates two related parts of the mitogen-activated protein kinase signaling pathway, or MAPKs - the extracellular-regulated protein kinases, or ERKs, and the stress-activated protein kinase (SAPK), also known as Jun N-terminal kinase (JNK and p38). Both of these systems are activated in response to kidney injury and/or renal tubular cell stress in vitro (464). Jun N terminal kinases (JNK and P38) are activated by inflammatory cytokines such as TNF-alpha and IL-1 and are only minimally activated by growth factors (464). JNK is activated in both proximal and distal tubules following ischemic injury (124). JNK down regulation ameliorates proximal tubule injury in response to peroxide induced injury (10)

ERK1/2 activity is typically activated by growth factor receptors, particularly, the EGFreceptor and other vasoactive molecules. The role of ERK in acute kidney injury is somewhat obscured due to conflicting results using different model systems. One point of view suggests that ERK activity is associated with cellular protection and facilitation of renal repair. Safirstein and colleagues demonstrated that ERK, but not JNK was activated in thick ascending limb cells following renal ischemia (464) and suggested that this was a means by which this nephron segment was protected from injury. Overexpression of ERK by adenoviral expression vectors in mouse proximal tubules resulted in protection from peroxide induced injury in vitro. When kidneys are protected via preconditioning, ERK activation is induced, while JNK activation is suppressed. Activation of the EGF receptor may be important in kidney regeneration (see below); activation of ERK via EGF receptor activity may convey protection to cells undergoing regeneration or to neighboring uninjured cells in a hostile environment. The non-specific MAPK inhibitor genestein blocked ERK induction following glycerol induced injury and this was associated with a less effective proliferative response (228).

In contrast, however, a significant literature exists demonstrating an important role for ERK activation in the induction of apoptotic cell death (reviewed in(646)). Inhibition of ERK activity blocks apoptosis associated with cisplatin nephrotoxicity in opposum proximal tubules in vitro (271). Pretreatment of MEK inhibitor, U0126, decreased ERK1/2 phosphorylation following cisplatin administration with significant functional and histologic protection (236). Inhibition of ERK can effect both intrinisic and extrinsic apototic pathways; ERK activation was shown to be upstream of caspase-3 activation (236). In 
response to cisplatin induced injury, inhibition of ERK may protect cells by repressing bax expression and/or inhibiting p53 activity $(78,206)$. The reason for the discrepant results remains unclear; it is likely that these are due to differences in injury models (i.e., cisplatin, hypoxia, reactive oxygen species) and the integration of down stream signaling pathways under different experimental conditions (e.g., JNK activation, growth factor activity, severity of injury).

IV.1.1.4. Other adaptive and reparative pathways: In addition to classic stress response pathways, additional pathways are activated in response to injury that may play an important role in stabilizing the injured kidney and/or promoting cellular repair processes.

The hypoxia-inducible factor-1 or 2, (HIF1 and HIF 2) are oxygen sensitive helix-loop-helix transcription factors that regulate biological processes which are geared toward facilitating oxygen delivery and/or providing cellular adaptation to hypoxia (183). This pathway is regulated by post-translational modification of HIF. Prolyl hydroxlase continuously modifies the HIF protein; this activity promotes the binding of HIF molecules to the pVHLubiquitin-ligase complex and directs HIF toward proteosomal degradation. Under hypoxic conditions, prolyl hydroxylase activity is reduced, minimizing the HIF degradation and allowing for HIF to interact with HIF response elements on a multitude of potential target genes (183). HIF target genes, if activated in AKI would clearly be beneficial to adaptation and repair, such as HO-1 and VEGF.

HIF1a/ HIF $\beta$ dimerization and nuclear translocation has been reported in kidneys following ischemic ischemic injury (139), and in response to hypoxia by vasoconstriction or anemia and by cobalt chloride. HIF1 has been observed in tubular epithelial cells while HIF2 staining was observed in endothelial cells $(137,138)$. In cultured proximal tubule cells, HIF1 competent cells are more resistant to hypoxic injury than HIF null cells due to increased glucose uptake capacity in HIF-competent cells (51); however, since proximal tubule cells in vivo do not utilize glucose primarily, the importance of this observation is unclear. Recent studies have demonstrated that both HIF1a and HIF2a heterozygote mice or HIF2 null mice manifest more significant injury than corresponding wild type mice in ischemia reperfusion injury (205). Strategies to induce HIF-1 activity have also been evaluated; prolyl hydroxylase enzyme inhibition results in renal HIF-1 activation with significant preservation from injury (205). Similarly, Morg1 (MAPK organizer 1) an enzyme that interacts prolyl hydroxylase, was evaluated in transgenic animals; HIF activation was increased and renal damage attenuated in Morg1 heterozygote mice relative to wild type controls (189). Although this latter report describes a reduced induction of inflammatory factors, the HIF target genes that mediate these protective response have not been well-delineated.

\section{IV.2. Preconditioning and pharmacological maneuvers of cellular protection}

Preconditioning is a powerful experimental maneuver that reproducibly protects the heart against subsequent ischemic challenge $(41,107)$. The concept of preconditioning applies readily to the kidney. Multiple studies carried out by several groups have demonstrated that prior stress or injury to the kidney provides resistance to subsequent injury (59).

Interestingly, preconditioning effects by nephrotoxic agents were reported by MacNider as early as the 1920s (343).

Preconditioning has been studied using several experimental approaches. For example, an "early window" preconditioning occurs in response to one or several bouts of short ischemia (varying between 4-15 minutes), a short recovery time (on the order of a few hours) followed by longer bout of ischemia that would be considered injurious (e.g., 30-60 minutes). Several examples of early-window preconditioning in the kidney exist $(291,555)$. 
It is typically interpreted that this preconditioning relies heavily on non-genomic activity to provide protection. Evidence suggests that preconditioning increases renal NO production leading to preservation of renal perfusion $(233,613)$. Other evidence suggest that the rapid activation of adenosine A1 receptors may mediate the preconditioning effect as A1 agonist treatment mimicked the pre-conditioning response, while an A1 antagonist prevented the preconditioning effect (291). Activation of the adenosine A3 receptor subtypes worsened renal injury.

"Late" window preconditioning refers to a process in which kidney injury is induced and is resistant to a second challenge during or shortly after the normal recovery response. The protective effects can been seen even very early during the repair phase of injury, i.e., within 1 day (636). The effect can also be prolonged; using a model of glycerol-induced injury, Nath et al., demonstrated protection from a second insult up to one month following recovery from the first injurious stimulus (394). In a mouse model of ischemia reperfusion, Park et al observed protection to a second injury between 5 and 14 days following recovery, while the protective response was gradually reduced between 14 and 28 days of recovery (424). The preconditioning effect may depend on injury in general and not the specific nature of the insult since other types of injuries induced by either cisplatin or transient ureteral obstruction can also have prolonged protective effects $(122,197,203)$.

The time course of resistance following such injuries suggests production of adaptive molecules may convey protection. A substantial number of genes are thought to be modulated by the preconditioning process (174). These include the afformationed stress response genes such as HSP25, HSP 72, p38 MAPK, HO-1, and HIF-1 (59, 124, 393, 425, $568,615)$ which may play a modulatory role in protection. Interestingly, injury itself may not be required; repetitive bouts of systemic hypoxia, in and of itself not injurious, can increase the expression antioxidant genes, such as SOD-1 or invoke anti-apoptotic genes and protect against $\mathrm{I} / \mathrm{R}(88,615)$. Alterations in cellular lipid composition are also present in the preconditioned state. A consistent finding secondary to multiple models of acute injury is the accumulation of cholesterol due to new synthesis of HMG CoA reductase $(632,635)$. Blockade of the HMG CoA reductase gene was shown to abrogate the cytoprotective effects of ischemic preconditioning (637).

Several strategies have been attempted to tap into the powerful protective effects conveyed by preconditioning. One strategy derives from the what is termed "remote" preconditioning, which refers to a process where injury in one tissue conveys protection at a distant organ site (449); this phenomenon suggests that circulating factors derived from ischemia may convey the protective effects. This cross talk appears to apply to injury in multiple organ systems; for example, short term ischemia in the kidney protects subsequent myocardial ischemia and these effects can be inhibited by adenosine A1 antagonists and mitochondrial KATP channel blockers (196). Conversely, liver injury can protect against subsequent injury to the kidney (17). One potential strategy might involve the induction of transient limb ischemia which has remote effects on preserving endothelial function (339).

A second strategy involves the activation of stress response pathways by various chemical and pharmacological agents. Preconditioning effects can be mimicked by multiple agents such as anesthetics like xenon, which activates ERK or HIF pathways (341). Inhaled CO and administration of a CO-releasing compound protect against AKI or transplant associated injury $(396,575)$. Several other factors that potentially influence stress response pathways and have protective effects include glutamine (157), erythropoietin (616), geranylgeranyacetone (535) and tin protoporphryin (243). 


\section{IV.3. Regeneration}

The restoration of kidney structure following injury has been the subject of substantial investigation. Evidence of recovery responses in the post injury period has been observed in patient biopsies following ischemic or nephritic injury or in biopsies from patients with delayed graft function $(447,511,514,515)$. In both rats and mice, severe acute injury following ischemia or nephrotoxic insults results cellular proliferation as shown by ${ }^{3} \mathrm{H}-$ thymidine $(109,110,361)$ or BrdU incorporation $(219,325,369,370,520)$ or by the expression of PCNA (360) or Ki67 antigen (481) usually apparent in less than 1 day following injury. The vast majority of this proliferation is present in the proximal tubule cells.

In addition to cell proliferation, the restoration of proximal tubule structure and function requires a variety of complex and well coordinated cellular activities (See Figure 11) (402). For example, in the early post-ischemic period, gaps of naked basement membrane can be found from which necrotic or desquamated cells have detached. Newly formed proximal tubule cells are thought to migrate rapidly to realign the proximal tubular basement membrane (402). These newly formed proximal tubule cells are dedifferentiated and have a very different gene expression profile vs. fully differentiated proximal tubules. Typical markers of epithelial cells such as cytokeratin and E-cadherin are lost and there is expression of markers of undifferentiated cells, such as vimentin (608). In renal injury models using young healthy rodents, the proliferative response is more robust than is required to replenish the proximal tubule. Shimuzu and Yamanaka demonstrated that the post mitotic, postischemic rat kidney have proximal tubules with an increased number of smaller tubular cells per cross sectional area than is observed in an uninjured kidney (497). Occasionally, these surplus cells can be observed to form papillary structures evaginating into the lumen of proximal tubule (497). These hyperplastic epithelial cells begin to take on the appearance of a normal proximal tubule cells by hypertrophy and differentiation and this is manifested as a decrease (back toward control levels) in the number of nuclei per cross sectional area (497), the loss of vimentin staining and the reappearance of differentiation markers (402). Concomitant with this process is a wave of apoptosis that restores normal tubular cell density that may last from between 1 week to several months of recovery (497).

\section{IV.3.1. Proliferation of proximal tubules and potential role in recovery-The}

peak of proliferative activity coincides in time closely with the severity of injury, suggesting that the induction of proliferation represents an intrinsic defense mechanism. Cultured proximal tubule cells proliferate in response to a wide variety of mitogens such as epidermal growth factor (EGF) (403), hepatocyte growth factor (HGF) (523), insulin-like growth factor-I (IGF-1)(54), and fibroblast growth factors (FGFs) (639).

A significant advance was realized in a landmark paper published 1989 by Humes et al, investigating the effect of exogenous EGF on the course of kidney injury in response to ischemia reperfusion in rats. Administration of EGF to rats at the time of ischemia reperfusion injury reduced the severity of the initial injury measured by serum creatinine, and hastened the recovery back to levels of sham control levels (217). This was also associated with a significant increase in the number of mitotic proximal tubule cells in kidney of injured rats treated with EGF (217).

This finding spawned a number of similar studies investigating a variety of polypeptide growth promoting factors in various models of acute kidney injury, such as HGF (369) and IGF-I $(38,103)$. IGF-I was effective at ameliorating AKI when provided prophylyctically to rats or when administered up to 24 hours following ischemia reperfusion injury (371). Using a dog autotransplant model, supplementation of the graft storage solution and treatment of recipients with IGF-I protected the graft and hastened return to renal function (435). In 
addition to growth promoting effects, IGF-I increases renal blood flow in animals models and healthy human control subjects via a NO-dependent mechanism (207). When IGF-I was administered to late stage CKD patients it improved GFR, and was well-tolerated in most subjects (405). However, in a multicenter clinical trial, IGF-I failed to demonstrate any effect on recovery from renal function in patients with established AKI (208).

IV.3.1.1. Evidence for endogenous growth factors in renal repair: While the experiments described above provide proof of principal that growth factors may facilitate renal regeneration, the extent to which endogenous growth factor activity mediates repair response is less clear. For example, IGF-1 expression is enhanced in damaged and regenerating tubule cells (356). However, IGF-I knockout mice are not viable and there are no reports demonstrating a definitive role for this factor in the repair of the kidney (332). Similarly, while the molecular expression of hepatocyte growth factor (HGF) and its receptor c-met increase in kidneys in rat models of AKI (223) and HGF is elevated in the urine of patients with acute kidney injury (539), there is no definitive data supporting a role for endogenous HGF activity in proximal tubule injury models. Although it has been reported that a targeted mutation of the c-met receptor in collecting duct impairs recovery from transient ureteral obstruction and hastens the development of fibrosis (342).

Epidermal growth factor, EGF, is down-regulated in response to multiple different forms of $\mathrm{AKI}$, and in fact the EGF gene is one of the most sensitive genes to ischemia-reperfusion injury (467). Despite its down-regulation, pre-pro-EGF present in the distal nephron is processed to an active form where it may participate in the renal repair response (476). A related growth factor, HB-EGF (heparin binding EGF), is expressed in the proximal tubule and is associated with the outer surface of the cell membrane where it can interact with the EGF-receptor (c-erb-B) in a juxtacrine/autocrine fashion. Injury activates the proteolytic processing HB-EGF to liberate a soluble form of the molecule (470). The importance of a functional EGF receptor in kidney repair was established using waved-2 mice (wa-2), which have mutated EGF-receptors with ineffective tyrosine kinase activity and decreased receptor signaling. These mice manifest a significant impairment in structural and functional recovery from mercuric chloride induced AKI (594).

Fibroblast growth factors- FGFs represent a family of heparin binding growth factors with potent proliferative potential of proximal tubules. Various members of the FGF family are induced following the induction of ischemic and nephrotoxic injury. FGFs share a series of common receptors; recent studies using local administration of antisense oligonucleotides against the FGF receptor showed a moderate effect on the expression of nephrogenic genes following ischemia reperfusion (576). Although not clearly established, Klotho, a senescence related molecule appears to interact with various FGFs; Klotho facilitates the binding of FGF23 to the FGF-R1 in kidney (565). Renal Klotho expression is suppressed following AKI, but adenoviral gene transfer of Klotho is cytoprotective in AKI (526)

BMP-7 has received considerable attention for its role not only in stimulating cell proliferation, also as a potential anti renal fibrotic agent in chronic kidney disease. Exogenous BMP-7 hastens recovery from AKI (579). Evidence for the involvement of endogenous BMP-7 in renal regeneration derives from studies using USAG-1 (uterine sensitization associated gene-1) knockout mice. USAG-1 is a powerful endogenous antagonist of BMP-7 and it is prominently expressed in kidney. USAG-1 -/- mice are resistant to AKI and have enhanced recovery following cistplatin AKI (614) These protective effects were abolished by the administration of BMP-7 neutralizing antibodies (614). 
IV.3.1.2. Immediate early genes and cell cycle signaling: In most models of renal injury there is a rapid increase in immediate-early gene expression. Activation of immediate early genes such as JE, KC (463) and Cyr61 have been reported (387), which may participate in early growth and or inflammatory processes. In ischemia, c-fos mRNA peaks as early as 1 hour and declines within 4 hours (463). Other "immediate early genes" associated with growth responses are also induced following injury including c-jun, c-myc and Egr1 (414, 463). These genes encode transcription factors that regulate growth processes suggesting a potential role in regeneration.

Injury and cell stress activate downstream signaling pathways associated with cellular regeneration conveying prosurvival signals. The EGF-receptor (EGF-R) is activated not only in response to EGF-related ligands (as described above) but other factors depending on the nature of the injurious stimulus. For example, following replating of tubule cells, EGF receptor activation and subsequent cell proliferation depends on the release of the HB-EGF ectodomain by the metalloprotease ADAM17 (645). In contrast, in response to oxidative stress induced by $\mathrm{H}_{2} \mathrm{O}_{2}$, EGF-receptor activation and subsequent cell proliferation is dependent on the activation of $\mathrm{Src}(645)$.

Cell proliferation in response to injury begins with entry into the G1 phase of the cell cycle, typically under the stimulation of extracellular signals derived from growth factors or integrin-linked kinase receptors. The coordinated synthesis and degradation of cell cycle inhibitors regulate important checkpoints within the cell cycle to ensure the successful completion of cell division (468). One cell cycle inhibitor of significant interest in the renal regeneration response is p21. p21 is a cyclin dependent kinase inhibitor, which controls the $\mathrm{G} 1$ to $\mathrm{S}$ phase checkpoint. Expression of p21 is associated with terminal differentiation and senescence of many cell types, but it is rapidly induced in the kidney following various forms of acute injury in such models as cisplatin or ischemia reperfusion $(360,362)$. p21 -/mice demonstrate a more rapid and pronounced level of renal injury when compared to wild-type mice, characterized by elevated BUN, and necrotic death even extending into the cortical S1 and S2 segments, and increased apoptosis $(360,362)$. Increased BrdU labeled nuclei were found in kidneys of p21-/- mice, however many of these cells had a polyploidy DNA content, suggesting that the lack of p21 regulatory control results in aberrant repair due with an uncontrolled cell cycle $(360,362)$.

IV.3.2. Migration and extracellular matrix-Severe renal injury leaves behind denuded areas of the basement membrane and also activates a variety of proteases with the potential to degrade the tubular basesment membrane. Studies by Zuk et al., (649) and by Walker (585), demonstrated a decrease in the collagen content and laminin content in the early postinjury phase, which is gradually restored over the course of several days of recovery. Given the close association between matrix and cell adhesion, cellular and matrix remodeling events are likely well coordinated, but are certainly poorly understood.

It is likely that the re-establishment of the basement membrane is critical to the reestablishment of proximal tubule function for proper presentation of integrin binding. There is increased expression of basement membrane ECM components by tubule epithelial cells such as collagen IV and fibronectin mRNA during the regeneration process following AKI $(37,650)$. An alternative splice variant of fibronectin (EDA, EIIIA) is expressed in the regenerating kidney in a TGF-beta dependent fashion (37); this form of fibronectin may modify cellular integrin binding and affect adhesion and cell migration (194). The significance of these findings on renal repair in vivo has yet to be established. To date, the primary method to address potential migratory activity utilizes migration assays to scrape injury in vitro. Using these approaches, plasminogen activator and EGF promote migration 
and repair of wounded monolayers of proximal tubules, while TGF-beta inhibits this process (402).

IV.3.3. Recapitulation of a renal development-During renal development, the formation of a morphologically polarized epithelium from committed renal mesenchymal cells requires an external signal for mitogenesis and differentiation (188). Many cellular events are common in both renal development as well as renal regeneration such as induction of growth factor activity, high rates of DNA synthesis, apoptotic remodeling, the production of ECM, and the interaction of surface receptors leading to an early organization of actin cytoskeleton. The plasma membrane organizes to define apical and basolateral domains and the formation of cell-cell contacts results in a polarized epithelium. These similarities prompted Bacallao and Fine to hypothesize that kidney regeneration utilizes an intrinsic developmental program as a basis for recovery from kidney injury (20).

Although difficult to prove, the notion that renal regeneration recapitulates development is supported from a variety of molecular/genomic studies demonstrating that the a substantial complement of genes typically restricted to renal embryonic development are re-expressed during kidney regeneration. As mentioned above, vimentin, is expressed in regenerating epithelia as well as developing epithelia during fetal development (28). However studies examining specific gene pathways as well as studies employing mRNA expression profiling have revealed a substantial number of kidney developmental genes expressed during renal regeneration; these genes include those involved in growth regulation, extracellular matrix factors such as FN EIIIA, transcription factors Pax2 and Wnt-4 $(225,540)$.

Epigenetic mechanisms including histone acetylation play a key role in organ development and cellular homeostasis by regulating gene expression in a tissue- and developmental stage-specific manner (530). Increasing histone acetylation, the levels of which are determined by the balance between the activities of histone acetyltransferase (HAT) and histone deacetylase (HDAC), generally stimulates gene transcription by relaxing the chromatin structure. Recent studies by Murano et al., suggest that modification of HDAC activity may provide an important regenerative feature of damaged kidneys. In cultured proximal tubules subjected to energy depletion, there was a decrease in histone acetylation and HAT activity. During recovery, the HDAC isosyme HDAC5 was downregulated as histone acetylation returned. Reduced HDAC 5 levels were associated with an increase in BMP7 levels. In vivo, HDAC expression was repressed in regenerating tubules on the outer medulla of post-ischemic mice kidneys, where BMP-7 levels were elevated (352)

In addition to effects on potential repair mechanisms, it has recently been shown that the transcription factor ATF3 interacts directly with histone deacetylase 1 (HDAC1) and recruits HDAC1 into the ATF/NF- $\kappa$ B sites in the IL- 6 and IL-12b gene promoters. Based on studies using ATF1 knockout mice, this interaction results in the condensation of chromatin structure, resulting in the interference of NF- $\kappa \mathrm{B}$ binding, and inhibition of inflammatory gene transcription after I/R injury (309).

The epigenetic control of gene expression regulating injury and repair processes in the setting of AKI is likely to be the source of significant new research findings in the coming years. Such advancements may likely provide a new viewpoint by which to understand events underlying remodeling occur. As suggested by Surendren and Kopan, the flexibility of histone remodeling may explain how various cell types can alter their differentiation status and re-enter the cell cycle, restore normal morphology, and provides a basis for regenerative processes to occur without a requisite requirement for stem cell niches (530). 
IV.3.4. Tubular cells comprise the majority of the regenerative cells during repair-Interest in stem cells as possibly participating in kidney regeneration derives historically from observations in kidney transplant patients where host cells have been reported to incorporate into the renal tubules of the graft (181). Thus, populations of circulating stem cells, presumably bone marrow derived, could home to the kidney and participate in renal recovery. However, the identification of these cells provides little insight into their participation in overall kidney repair.

Some reports have suggested that BM derived cells may transdifferentiate into tubules or may fuse with endothelial cells following injury $(82,324)$. However, the proportion of bone marrow derived cells that integrate stably into tubular epithelial cells or endothelial cells is small. In a study by Lin et al., a transgenic cre-lox approach was used to label kidney epithelial cells and track their progeny following I/R injury. They demonstrated that BrdU was localized in dedifferentiated cells that expressed the Cre-induced transgene, suggesting that the source of regenerating cells was resident, non lethally injured epithelial cells (Figure 12). In the same study, this group demonstrated transplanted bone marrow GFP+ cells localized to the kidney interstitium one month after injury, but not to renal tubules (325). Other groups have subsequently reaffirmed that the primary source of new cells during regeneration is sublethally damaged kidney tubule cells (219), while no studies using bone marrow transplants have demonstrated that such cells comprise a significant source of regenerated epithelium. Thus, there is little remaining controversy regarding the source of new cells during the regenerative process post-AKI. However, as described below, it remains possible that circulating stem cells may augment renal repair mechanisms and that stem cell niches within the kidney may participate in regeneration.

IV.3.5. Potential contribution of bone marrow derived stem cells-Despite the lack of convincing evidence that bone marrow derived cells contribute to the formation of new cells during renal repair, recent studies clearly suggest a beneficial role for bone marrow derived cells in aiding the regeneration process and provide a possible therapeutic modality. Two distinct stem cells reside in the stroma of the bone marrow; the hematopoietic stem cell (HSC), undifferentiated cells capable of self renewal and giving rise to the specialized cells of the blood including, erythrocytes, thrombocytes and leukocytes. Mesechymal stem cells (MSC) are of mesodermal origin and are characterized by the expression of CD90, CD73, CD104, CD44 and CD29 but do not express markers of hematopoietic lineage, such as CD34 and CD45 (82). These multipotent cells give rise to cells of connective tissue such as bone, fat, cartilage and muscle.

IV.3.5.1. Hematopoetic stem cells in renal repair: Generally speaking, HSCs are not thought to contribute prominently toward a repair process in the setting of AKI. Some reports have demonstrated that HSC may incorporate into and differentiate into renal tubular cells following injury, however the number of cells of this nature is quite small (324). Typically, administration of undifferentiated HSC or their mobilization worsens kidney injury by exacerbating the extent of renal inflammation $(549,550)$. In contrast, other reports have demonstrated a beneficial effect of HSC mobilization repair (230). The reason for the discrepant reports is unclear but may relate to the specific methodologies used to induce mobilization.

In addition, HSC may also give rise to a population of cells referred to as endothelial progenitor cells, or EPC. EPCs represent a population of cells derived from HSC which express proteins common to endothelial cells (e.g., VEGFR-2 and V-cadherin), but also markers of hematopoietic lineage (e.g., CD34 and CD133) (455). There is significant confusion in the literature regarding the nature of these cells as the term EPC is likely a misnomer. These cells do not give rise to mature endothelial cells and do not stably integrate 
into blood vessels. However, they likely do help facilitate vascular growth and repair processes and therefore may be properly termed "angiogenic" macrophages (623).

Regardless of the terminology, these cells can home transiently to sites of vascular injury and can produce pro-angiogenic factors thought to aid in the angiogenic process and influence mature endothelial cells $(455,623)$. Several clinical studies suggest that host EPC may migrate and incorporate into tissue grafts following renal transplant (455) but the importance of this observation on maintenance of renal function and repair is not known. In a mouse model of ischemia reperfusion injury Patschan et al, demonstrated that ischemic preconditioning increased the deposition of CD34+/Flk+ cells which are also positive to c$\mathrm{kit} / \mathrm{and} \mathrm{Tie} 2$. When these cells were isolated and administered to naïve mice, they preserved renal vascular function in response to ischemia reperfusion (426). Recently, Li et al., demonstrated the human CD34+ HSC accelerated recovery following I/R injury in immunocompromised mice (307). Administration of factors such as erythropoietin or uric acid may serve as a trigger to increase the number of EPC migrating to the kidney and have a beneficial effect on vascular injury $(209,427)$.

IV.3.5.2. Mesenchymal stem cells in renal repair: In contrast to HSC, MSC have received considerable attention in the setting of acute kidney injury. MSC do not elicit immune responses, may be harvested from non bone marrow depots (such as adipose) and can be expanded in vitro making them potential useful therapeutically $(82,557)$. Several groups have reported a beneficial effect of MSC to experimental animals following AKI. MSC treatment provides protection when administered at the time of injury and hastens repair after established AKI induced by I/R $(385,553)$. Despite the differentiative potential of MSC there is no evidence to suggest that these cells integrate stably into the repairing kidney. Togel et al., demonstrated that MSCs traffic only transiently in the post-ischemic kidney declining within 1 day with the majority of cells homing in lung and spleen (552). Therefore, it is suggested that the reparative effects of MSC is via the production of endocrine or paracrine factors. For example, MSC produce high amounts of VEGF and IGFI, which when repressed via siRNA impair MSC effects on recovery from AKI (551). It is likely that increasing MSC homing will enhance the regenerative properties provided by MSC. Following glycerol induced injury, MSC homing was suggested to be due to CD44 interaction with hyluronic acid as inhibition of CD44 on transplanted cells resulted in less homing and less repair (204). Homing to the kidney may not required at all; Lee et al., have recently demonstrated that MSCs after homing to lung activate the production of the antiinflammatory peptide, TSG-6, which facilitates recovery from myocardial ischemia (293). Recently, clinical trials with promising potential have been initiated and may represent the advent of a novel therapeutic avenue in the treatment of AKI (553).

Whether endogenous MSC might participate in renal repair has not been adequately addressed. However, recent studies from Goligorsky's group have identified a population of MSC that lie within the renal capsule. Cell-tracker labeling in vivo demonstrated that these cells have the ability to proliferate and preferentially home to the ischemic perivascular space in the outer medullary region within 3 days of I/R injury. Decapsulation of the kidney modestly attenuated the recovery from renal injury suggesting a partial role for endogenous MSC in renal repair (423).

IV.3.6. Renal derived stem and progenitor cells-Other investigations have been directed toward the concept that renal repair is mediated via intrinsic renal stem and/or progenitor cells following injury. Stem cells, by definition are clonogenic cells capable of both self renewal and multilineage differentiation. In contrast, progenitor cells are defined as immature and proliferative cells which are limited in their differentiation potential to only one cell type (460). A lack of clear markers on what defines a population of renal stem/ 
progenitor cells, as well as the possibility that there are likely multiple populations of cells with progenitor activity have contributed to some controversy in this area. To date, several different stem and progenitor cells have been identified and may participate in kidney repair in animal models.

For example, Bussolati et al., have isolated cells from human and animal kidneys expressing CD133, a well accepted stem cell marker, using magnetic sorting techniques $(81,82)$. These cells lack hematopoietic markers but express the renal developmental transcription factor, Pax-2. These cells also show limited self renewal and could differentiate into either epithelial or endothelial cells in culture $(81,82)$. Administration of these cells into SCID mice with glycerol induced tubular injury resulted in their homing and their integration into tubular structures $(81,82)$. However, there is no direct evidence that CD133 expressing cells within the kidney participate substantially in repair in response to an injury.

Gupta et al., isolated a unique population of cells from adult rat kidney cells that displayed features of a multipotent renal progenitors (termed MRPC). These cells exhibit self renewal and expression of vimentin, CD90, Pax 2 and Oct 4 without evidence of senescence. Oct4 expression was seen in some tubular cells in the adult kidney, suggesting these cells may be candidate renal stem cells. The cells can differentiate into renal tubules when injected under the capsule of an uninjured kidney or intra-arterially after renal ischemia-reperfusion injury (182). Again, evidence that endogenous MRPC participate in the injury process has remained elusive.

Finally, a population of progenitor cells has been identified in the renal papilla based on their ability to retain BrdU label when administered during the early embryonic or neonatal development $(345,411)$. These "label retaining cells" (LRC) cells can be isolated and expanded in culture and display activity consistent with stem cells as they give rise to multiple different cell types $(345,411)$. There are multiple conflicting reports on the fate of LRC that may be based in part on different protocols used to identify them. In one report, Maeshima infused BrdU into rats for 7 days followed by 2 weeks of chase. Subsequent injury by ischemia reperfusion resulted in a transient increase in the number of LRC many of which were dedifferentiated and expressed markers of proliferation (346). In contrast, when LRC were labeled in mice by embryonic BrdU administration and studied at 2 months of age, LRC were found in the outer stripe of the outer medulla but most Ki67 cells did not colocalize with these LRCs (269).

IV.3.7. Impaired recovery in aging and CKD-Elderly patients are more prone to develop AKI and to have a less successful outcome $(93,227,479,480)$. It needs to be emphasized that most of our understanding of the reparative responses of the kidney derive from studies on young healthy animals and such mechanisms are likely not as efficient in older animals (479).

Studies in rats and mice have demonstrated an impaired recovery response in older animals vs. younger animals $(89,481)$. The increased susceptibility is likely the result of multiple factors including impaired vascular reserve, as well as an impaired regenerative capacity. In older animals in response to injury, less cell proliferation was observed and the intrinsic growth potential (i.e., colony forming potential) of proximal tubule cells was significantly reduced in older animals vs. younger animals (481). A comparison of gene expression demonstrated that tubules from older animals express greater amounts of zinc a 2 glycoprotein-1 (Zag-1), a putative inhibitor of proliferation (481). Chen et al (89) demonstrated that the age related sensitivity to injury could be ameliorated by caloric restriction. Up to 92 different genes were altered in the aging kidney that were attenuated by with caloric restriction including genes associated with tight junction activity (Claudin-1) 
and genes associated abnormal matrix regulation, such as MMP-7. Aging likely influences a number of processes regulating epithelial growth potential and/or senescence.

Shortened telomeres are associated with cell senescence and the biological significance of this in recovery from AKI has been studied in a mouse model of telomere shortening. Mice with null mutation in telomerase gene (Terc-/-) develop significantly shortened telomeres within 4 generations and have increased sensitivity to AKI. Moreover, these mice manifest a less successful recovery response that is associated with interstitial fibrosis. At the molecular level, there is an increased expression of the cell cycle inhibitors p21 and p16INK4a relative to age matched wild type controls (604). Interestingly, the cell cycle regulator p16 ${ }^{\mathrm{INK} 4 \mathrm{a}}$ is highly expressed in aging human kidney; following renal transplant, its expression is elevated in failing renal grafts while it low transplant with immediate function (364).

Impaired recovery from renal damage may also be a function of pre-existing renal disease. Epidemiological studies suggest a less successful recovery of individuals from AKI with established CKD (227). Although early impairment in regional blood flow is worsened in diabetic $\mathrm{db} / \mathrm{db}$ mice, there is a paucity of studies investigating renal repair potential in models of CKD (496). However, several studies have investigated recovery of AKI in conjunction with reduced renal mass. Such studies show evidence of impaired recovery characterized by incomplete return of GFR and impaired differentiation of regenerating proximal tubules (617). As described below, impaired recovery may relate in part to the predisposition of chronic kidney disease following AKI.

\section{IV.4. Chronic effects of AKI}

Incomplete repair or conversion from physiological to a pathological repair response could result in declining renal function and chronic kidney disease following AKI. Although typically AKI is not thought to predispose the development of long-term kidney complications, it has been known for several decades that many adult patients who suffer from AKI never recover renal function completely, manifest decrements of GFR and have impaired urinary concentrating ability $(58,71,147,275,304)$. Pediatric patients have a particularly high risk of developing CKD and hypertension following AKI $(8,16)$. As the incidence of AKI has risen, so too has interest in the long-term sequelae of AKI and several studies now suggest patients with AKI progress to $\operatorname{ESRD}(9,97,227,543)$.

From a pathophysiological perspective, animal model data support the connection of AKI with CKD and offer potential mechanistic insights into the process. Nath et al., used a model of cutaneous glycerol injection to induce AKI resulting in a significant but reversible decrease in creatinine clearance in rats. Repetitive administration of glycerol (once monthly over 6 months) resulted in a slow progressive loss of GFR associated with increased TGFbeta expression and progressive interstitial fibrosis (394). The development of CKD occurred despite the presence of an adaptation to the subsequent repetitive glycerol injections, (i.e, preconditioning) suggesting a disconnect between the acute and chronic manifestions of this insult. Moreover, the results imply that CKD may result from a series of small repetitive acute injuries that may not manifest clinically as AKI.

Single episodes of AKI have the potential to alter renal function on a permanent basis. Chronic reductions in urinary concentrating ability have been observed in post AKI rats following either cisplatin or ischemia reperfusion induced injury $(32,465)$. Although early elevation in diuresis may be due to increased $\mathrm{Na}$ excretion or impaired aquaporin activity, concentrating ability remains chronically impaired even following the return of \%FENa to sham levels and the resolution of the response to dehydration stress. Rather it was suggested that the urinary concentration defect persisted due to impaired ability of the kidney to generate a hypertonic medullary gradient $(32,465)$. 
Following a single bout of ischemia reperfusion, proximal tubule repair is typically complete within $4-6$ weeks in rats. The renal expression of TGF- $\beta$ and its cognate receptors are elevated transiently and return to control levels within approximately 4 weeks. With increasing recovery times (16-40 weeks), features of CKD ultimately become manifest, including proteinuria, interstitial fibrosis associated with a secondary increase TGF- $\beta$ (32).

Renal regeneration is less complete and progression of CKD more rapid and severe when AKI is carried out in the setting of reduced renal mass $(31,167,416,574)$. In an elegant study by Patgulanan et al., morphometric analysis of ultra thin serial sections revealed AKI resulted in nephron dropout, presumably due to loss of contact between the injured tubule and its parent glomerulus (416). Post-AKI kidneys in reduced renal mass models have a high proportion of dilated tubules containing cells which have not undergone redifferentiation. Venkatachalam et al., have recently focused attention on the existence of dilated tubules containing dedifferentiated epithelial cells and suggested that these may play a central role in the conversion AKI to CKD. According to the hypothesis, dedifferentiated cells express soluble profibrotic factors which act initially in the physiological repair response; however sustained expression of such factors (e.g., PDGF) may switch the normal repair process to a pathologic/fibrotic response (574). It is possible this relates to the recent observation by Yang et al., in which cells that are growth arrested in G2/M express high levels of profibrotic cytokines such as TGF-beta and CTGF (617). It is currently unclear why dedifferentiated cells are more prominent when regeneration occurs in the presence of reduced renal mass. One possibility may rest with a persistent expression of the TGF-beta axis, which may inhibit redifferentiation of proximal tubule cells (167). However, contributory factors related to reduced renal mass such as exacerbated hypoxia, increased SNGFR and/or exhausted renal compensatory reserve are additional possibilities that remain to be explored. Epigenetic factors may influence gene expression in the post injury state; in a recent report, Zager and colleagues demonstrated that histone modifications may be responsible for sustained profibrotic gene transcription leading toward CKD following an I/ $\mathrm{R}$ insult (636).

\section{IV.4.1. Impaired vascular recovery following AKI: a possible contributor} toward CKD—Although total renal blood flow typically returns to baseline values between 1-4 weeks of recovery, our laboratory hypothesized that there is chronic impairment of medullary vascular function following AKI. This hypothesis was based on early pathological observations suggesting vasa recta capillaries from post AKI patients were separated from nearby cells of the loops of Henle, potentially compromising the efficiency of the processes driving the generation of the medullary concentration gradient. Using microfil to generate vascular casts, we demonstrated between a 30-50\% reduction in the total number of microvessels, most pronounced in the inner stripe of the outer medulla, after recovery from AKI (Figure 13) (32). It is important to note that such alterations in renal blood vessel density do not necessarily indicate a reduction in total RBF and GFR as these values were typically restored to normal levels when vessel density measurements were made $(102,250,629)$. Recently published studies have shown reductions in peritubular capillary density in transplanted renal grafts; in these studies, the degree of capillary rarefaction is more pronounced in grafts experiencing delayed function $(283,510)$.

These observations indicate that the regenerative capacity of the vasculature is reduced relative to that of the tubular epithelium; this impaired vascular regeneration may predispose CKD via a number of possible mechanisms. Hypoxia is a well-known contributor to interstitial fibrosis and is thought to directly stimulate the expression of genes with profibrotic activity (404). Recovery from AKI was shown to be associated with persistent hypoxia using the 2-pimonidizole technique. AKI in combination with reduced renal mass (unilateral nephrectomy) exacerbated hypoxia and accelerated the development proteinuria 
and interstitial fibrosis. Chronic L-arginine supplementation beginning after the establishment of injury, did not protect microvessel dropout, but did increase renal blood flow, reduced hypoxia and attenuated the development of interstitial fibrosis and proteinuria (31).

In addition to its role in hypoxia, reduced medullary blood flow is a characteristic of many models of hypertension (104). Johnson and Schreiner suggested a cause and effect relationship between peritubular capillary loss and salt sensitive hypertension in response to various acute insults (239). When post-AKI rats are provided elevated dietary salt after the completion of the initial recovery response (5 weeks), hypertension develops along with substantial interstitial fibrosis (519). There were no differences between post AKI and sham animals in terms of plasma renin activity or circulating Ang II levels. However, when normotensive post-AKI rats were analyzed acutely after 5 weeks of recovery, there was a rightward shift in the acute pressure natriuresis relationship. In addition, there was impaired medullary flow and interstitial hydrostatic pressure responses to elevations in renal perfusion pressure (429). Thus, impairment of the renal microvasculature represents a primary cause of salt-sensitivity in post AKI rats.

Administration of VEGF-121, a non-heparin binding form of VEGF, can attenuate the loss of renal microvessels, and/or may induce new vascular cell proliferation in various models of progressive renal fibrosis such as aging or reduced renal mass via 5/6 nephrectomy (247, 248). VEGF-121 administration did not affect the initial loss of renal function (i.e., serum creatinine) following bilateral ischemia reperfusion injury, but did mitigate the loss vascular density measured at 5 weeks of recovery. VEGF 121 treatment ameliorated the secondary salt sensitive manifestations of CKD following AKI (302). Importantly, VEGF-121 did not protect against CKD when administered after 3 weeks of recovery, suggesting that there is an effective window of opportunity for intervention in the early injury/repair process. A similar protective effect on capillary preservation and subsequent fibrosis was observed in post-ischemic mice treated with cartilage oligomeric matrix protein/angiopoietin-1 (COMPAng1) a variant of the native angiogenic factor, angiopoietin 1 (242). Taken together, these data highlight the potential importance of the renal microvascular stability in the function of the post-AKI kidney.

\section{IV.4.2. The basis of vascular loss and impaired recovery following acute injury}

- There is no clear consensus regarding the cellular basis of vascular cell loss following injury, which complicates further our lack of understanding of suboptimal vascular repair responses. There is an early alteration in endothelial structure that promotes vascular leakiness (534), but it is not yet clear if this is related to permanent alteration in vascularity. It is certainly reasonable to suggest that apoptotic cell death underlies vascular (i.e., endothelial) cell loss following the induction of acute injury. Lee et al., demonstrated peritubular capillary endothelial cell apoptosis following severe liver I/R in mice which results in a subsequent AKI (292). However, Hörbelt et al., used Tie-GFP mice to label renal endothelial cells and were not able to identify apoptotic endothelial cells in response renal ischemia reperfusion, but did identify apoptotic endothelial cells in response to CD95 ligand. Therefore, it seems that the mode of cell loss (apoptosis or not) may be due in part to the nature of the underlying injury (212).

We recently provided evidence that endothelial mesenchymal transition, a process by which endothelial cells transform into a mesechymal phenotype, may contribute to vascular cell loss following AKI. There was a significant increase in cells that co-localize both endothelial markers (CD31 or cablin) and mesenchymal markers (S100A4) following I/R injury (35). Fate tracing of endothelial cells using Cre-Lox labeling indicated that interstitial 
cells observed 2 weeks following injury were of endothelial origin and the presence of these cells were reduced by treatment with VEGF-121 (35).

Finally, Lin et al, have posited that vascular loss may be mediated by a loss of the critical interaction of the endothelial cells with their neighboring perictyes in response to injury (328). In this hypothesis, the loss of trophic support from surrounding pericytes predisposes the loss of endothelial cells and results in impaired remodeling.

Regardless of the mode of cell loss, the persistent reduction in vascular density highlights the lack of regenerative capacity of renal vascular cells. The basis for the resulting lack of repair may stem from an impaired cellular responsiveness and/or combination with a lack of appropriate growth and/or trophic support factors and evidence exists for both possibilities. Indeed, in contrast to the enhanced expression of factors promoting the growth of epithelial cells, AKI induces a shift toward factors that may prohibit endothelial growth. TGF-beta is a negative regulator of endothelial proliferation and, in certain endothelial cell culture systems, induces endoMT. TGF-beta is prominently expressed during the regeneration process in ischemic and nephroxic AKI models (38). Inhibition of TGF beta using antibodies preserved vascular density and attenuated the deposition of peritubular fibroblasts (520). In addition, other inhibitors of endothelial cell growth are expressed following AKI including angiostatin, sFLT, ADAMTS-1, endostatin, arrestin and canstatin $(33,34,212)$.

Renal tubular expression of VEGF is thought to be the primary source of local vascular trophic support. Reductions in VEGF expression as a function of age correlate with vessel loss (544). Similarly, other models of CKD are characterized by reduced VEGF and loss of peritubular and/or glomerular vessels $(239,404)$. Because hypoxia is considered a primary trigger for VEGF expression via the HIF1a system (183), it is surprising that VEGF expression decreases rather than increases in developing CKD. Following AKI, VEGF expression has been reported to be either unchanged or transiently reduced $(33,246)$. It is possible that a transient reduction in VEGF expression may underlie vascular dropout since administration of VEGF-121 could attenuate vascular loss when administered within the first few days of ischemia (302). Although the balance of research done to date indicate an increase in vascular destabilizing factors and a decrease in pro-angiogenic factors following AKI, angiopoeitin 1, a vascular stabilizing factor was reported to be prominently and persistently induced in the mouse kidney following AKI (212).

Impaired vascular growth may also be due to impaired cellular responses to paracrine cues. Indeed, while new cell proliferation of glomerular endothelial cells has been demonstrated in the early stages of diabetic glomerulopathy or other models of glomerular injury $(151,249)$, there is little evidence that peritubular capillary endothelial cells undergo proliferation in disease states. In a recent study, no BrdU positive endothelial cells were detected within 2 days of ischemic AKI, and less than 1\% of BrdU cells labeled endothelia after 7 days of continuous BrdU administration. VEGF-121 preserved vessel integrity but did not influence endothelial proliferation (35); this suggests that VEGF protects via non proliferative mechanisms (e.g., perhaps blocking transition), but also suggests that renal endothelial cells have intrinsically poor growth potential. It is also noteworthy that endothelial cells of the kidney are notorious for being difficult to grow in culture. The difficulty in growing renal endothelial cells may reflect an intrinsic impairment of their growth potential relative to other endothelial cells derived from different vascular beds.

IV.4.3. Fibroblasts and myofibroblasts-AKI is associated with the deposition of interstitial fibroblasts or myofibroblasts, an important factor necessary for the development of interstitial fibrosis $(520,574)$. The source of these cells may be secondary to proliferation, transdifferentiation and/or migration or infiltration. Studies investigating the origin of renal 
fibroblasts can be complicated by the use of different markers for these cell types and controversy regarding their appropriateness. Nevertheless, some rather interesting concepts are worth summarizing.

The $\mathrm{Ca}^{++}$binding protein S100A4 has also been termed fibroblast specific protein-1 (FSP-1) and has been proposed as a marker of interstitial fibroblasts. In a classic series of studies by Neilson's group, overexpression of FSP-1 in cultured proximal tubules led to the transformation of these cells into a fibroblastic phenotype (408). This led to the suggestion that fibroblasts derive from epithelial cells via a process called epithelial mesenchymal transition (EMT), contributing to the development of interstitial fibrosis (245). Moreover, colocalization of FSP-1 with epithelial markers was subsequently considered suggestive, but not definitive evidence for EMT. A more definitive approach uses epithelial cells labeled with Cre-Lox approaches for fate tracing; When tubular epithelial cells are labeled with a reporter gene using Cre-Lox, ureteral obstruction resulted in interstitial expansion of labeled cells which stained positively for FSP-1 (229). However, other investigators using similar approaches have not been able to confirm these data (218), so the role of EMT in interstitial fibrosis represents a controversial area.

In ischemic AKI, a role for EMT is not well-supported by the literature. In the study described above by Lin et al., Cre-lox labeled tubular epithelial cells were shown to be the source of proliferating immature cells during regeneration. However, the study is also important as it demonstrated that labeled cells remained restricted to the tubule after the final recovery response and did not migrate to interstitium, strongly arguing against EMT (325). In contrast to epithelial mesenchymal transition, recent evidence from our laboratory suggested that endothelial mesenchymal transition (EndoMT) may not only contribute to vessel dropout, but also deposition of interstitial cells (35).

It is possible that fibroblasts/myofibroblasts derive from the circulation and target the injured kidney, perhaps to participate in physiological or pathological repair. Bone marrow transplant of transgenic animals expressing a trackable marker (i.e, beta gal, or GFP) demonstrate bone marrow derived cells in the interstitium following injury, some of which express S100A4 (75). These cells may be macrophages, since S100A4 expression has been described in this cell type. In contrast, these cells may also represent a form of a circulating cell type known as the fibrocyte (580).

An additional hypothesis recently put forward for the genesis of myofibroblasts was recently articulated by Humphries et al. in which a cre expression was driven in non SMA positive pericytes using the Col I promoter (218). Ureteral obstruction promotes SMA expression in these pericytes accounting for a large number of interstitial myofibroblasts. It will be of interest to see if other models of AKI are also associated with expansion and activation of pericytes as a prominent feature of fibrosis.

IV.4.4. Contribution of immune and inflammatory factors-It is clear that immune and inflammatory factors participate in the progressive process following AKI. Not surprisingly, a number of studies derived primarily from the transplant literature demonstrate that immune suppression blocks the expansiveness of nephropathy following an acute injury. A comprehensive assessment of the long term immune responses following $\mathrm{AKI}$ is beyond the scope of this review, however there are a few important points worth highlighting. An important role of both lymphocytes and macrophages has been demonstrated in progression following acute injury. Forbes et al, demonstrated that an early treatment with an endothelin antagonist blocked the deposition of ED-1 positive macrophages and attenuated the late manifestions of acute kidney injury (153). Secondly, there is clearly an important yet unexplained role for T cells in CKD development following 
AKI. In a classic study by Chandrakar et al, the B7 costimulatory pathway was blocked one month following unilateral $\mathrm{I} / \mathrm{R}$ with reduced renal mass, resulting in a dramatic decrease in the development of proteinuria and interstitial fibrosis with recovery times up to 6 months (86). Burne-Taney et al carried out adoptive transfer of splenocytes from post-I/R or shamoperated mice and followed their renal function over time. Splenocytes from injured mice promoted proteinuria non injured recipients within 12 weeks of injury, suggesting that immune responses were sufficient to mediate some of the abnormal responses (79). Finally, Pechman et al, blocked lymphocyte activity with mycophenolate mofetil beginning 5 weeks following recovery from I/R injury. This treatment prevented the development of hypertension and the dramatic increase in interstitial fibrosis concomitant with elevated sodium intake (428). Taken together, it would seem that the full manifestion of CKD following AKI relies on several important features working together including, improper tubule repair, vascular dropout, and persistent activation of immune function. Activities for progression are fueled by features such as reduced renal mass, elevated sodium intake and perhaps, even dietary proteins sources or underlying genetic factors. A schematic summarizing these events is shown in Figure 14.

\section{Conclusion}

Acute kidney injury remains a vexing problem in the clinical setting. The last four decades of research have substantially advanced our understanding of the cellular and pathophysiological processes in AKI and the recovery of organ function following established injury. Such work has provided an ample number of potential therapeutic targets yet effective therapies have remained elusive. What then, are the challenges in AKI research that should be addressed in the coming years? In our opinion, technological advances in genomics, proteomic and engineering will be at the forefront of exciting new work on the field of AKI. However, such technologies will likely be focused on addressing established problems not yet adequately addressed. For example:

1. Studies that will identify renal injury earlier and that will more precisely stratify patients based on the stages of injury will aid clinical and preclinical targeting of important mechanisms of kidney dysfunction in AKI. Factors controlling the initial fall in GFR may not be the same as those leading to sustained reductions in GFR during the extension and maintenance phases of injury and as described previously, the assessment of renal function based on surrogate markers of renal injury and GFR is fairly imprecise. Markers such as creatinine change slowly over time following injury to the kidney $(138,363)$. Improved methods for evaluating GFR and a more rapid assessment of renal injury are likely to improve the ability of the practicing nephrologist to diagnose AKI earlier and perhaps identify the clinical course more accurately (96). Based on our current understanding of the clinical phases of injury and repair processes, a more reliable charting of the course of individual patients may allow therapies tailored for each phase.

Over the past decade, significant advancements in the field of clinical proteomics have been targeted toward identifying novel plasma and urine proteins that may characterize the course of renal injury. The advent of the field of biomarkers and its potential impact on AKI is vast and a thoughtful consideration of its role in diagnosis and treatment options on AKI is beyond the scope of this chapter. However, it must be pointed out that potential markers such as NGAL, KIM-1, cystatin C, IL-18 and NAG have all been postulated to represent early markers of renal injury and the reader is encouraged to seek reviews dedicated to this topic $(138,422,504)$. Of note, NGAL, a protein that is upregulated in the proximal tubule following acute injury, and KIM-1, a membrane glycoprotein whose 
ectodomain is shed into the urine following renal injury, have received a significant attention and potential early markers of renal injury.

This field has moved rapidly in the last decade from identification of these molecules in the preclinical models, to the initial validation in limited clinical settings $(47,315,331)$. Although these studies suggest a potential predictive power, the next several years should provide important information on whether such tools will enable the clinician to initiate treatments earlier and to determine if stratification of patients based on such markers will provide any substantive impact in terms of outcome.

2. Studies that provide a better understanding of the link between tubular injury and the fall in GFR need to be performed. Crosstalk between tubules, blood vessels/ endothelium, inflammatory cells, and resident supporting cells may be key in defining this area and warrant further investigation.

3. A greater recognition of the simplicity of our preclinical models and a more precise understanding of the factors in AKI that lead to death, including but not limited to issues related to organ cross talk and the development of therapies geared toward sustaining multi organ function.

4. A continuation of work on recovery response of AKI will continue to be of significant interest, and important advances will likely be influenced by advances genomic and epigenetic technology. In our opinion, an understanding of the factors that lead from physiological repair to a pathological response leading to CKD will represent an increasingly important area; therefore, work leading to the identification of patients at high risk for developing AKI may help to improve long term outcomes. All in all there is still much work to be done. However, recent efforts provide optimism that some of the long standing hurdles to progress may soon be overcome.

\section{Acknowledgments}

The authors are supported by NIH grants DK063114 (DB), and DK-077124 (TS) and pilot funding from the George M O’Brien grant, P30 DK79312 (Bruce Molitoris, Program Director), and Bridge Funding from the Indiana University Research Foundation.

\section{References}

1. Abul-Ezz SR, Walker PD, Shah SV. Role of glutathione in an animal model of myoglobinuric acute renal failure. Proceedings of the National Academy of Sciences of the United States of America. 1991; 88:9833-9837. [PubMed: 1946409]

2. Adabag AS, Ishani A, Koneswaran S, Johnson DJ, Kelly RF, Ward HB, McFalls EO, Bloomfield $\mathrm{HE}$, Chandrashekhar Y. Utility of $\mathrm{N}$-acetylcysteine to prevent acute kidney injury after cardiac surgery: A randomized controlled trial. American heart journal. 2008; 155:1143-1149. [PubMed: 18513531]

3. Agarwal A, Balla J, Alam J, Croatt AJ, Nath KA. Induction of heme oxygenase in toxic renal injury: A protective role in cisplatin nephrotoxicity in the rat. Kidney Int. 1995; 48:1298-1307. [PubMed: 8569092]

4. Aki Y, Nishiyama A, Miyatake A, Kimura S, Kohno M, Abe Y. Role of Adenosine A1 Receptor in Angiotensin II- and Norepinephrine-Induced Renal Vasoconstriction. Journal of Pharmacology and Experimental Therapeutics. 2002; 303:117-123. [PubMed: 12235241]

5. Akira S, Takeda K. Toll-like receptor signalling. Nat Rev Immunol. 2004; 4:499-511. [PubMed: 15229469] 
6. Alejandro V, Scandling JD Jr, Sibley RK, Dafoe D, Alfrey E, Deen W, Myers BD. Mechanisms of filtration failure during postischemic injury of the human kidney. A study of the reperfused renal allograft. J Clin Invest. 1995; 95:820-831. [PubMed: 7860766]

7. Alejandro VS, Nelson WJ, Huie P, Sibley RK, Dafoe D, Kuo P, Scandling JD Jr, Myers BD. Postischemic injury, delayed function and $\mathrm{Na}+\mathrm{K}(+)$-ATPase distribution in the transplanted kidney. Kidney Int. 1995; 48:1308-1315. [PubMed: 8569093]

8. Alon US. Neonatal acute renal failure: the need for long-term follow-up. Clinical Pediatrics. 1998; 37:387-390. [PubMed: 9637905]

9. Amdur RL, Chawla LS, Amodeo S, Kimmel PL, Palant CE. Outcomes following diagnosis of acute renal failure in U.S. veterans: focus on acute tubular necrosis. Kidney Int. 2009; 76:1089-1097. [PubMed: 19741590]

10. Arany I, Megyesi JK, Kaneto H, Tanaka S, Safirstein RL. Activation of ERK or inhibition of JNK ameliorates H2O2 cytotoxicity in mouse renal proximal tubule cells. Kidney Int. 2004; 65:12311239. [PubMed: 15086462]

11. Argaud L, Gateau-Roesch O, Muntean D, Chalabreysse L, Loufouat J, Robert D, Ovize M. Specific inhibition of the mitochondrial permeability transition prevents lethal reperfusion injury. Journal of Molecular and Cellular Cardiology. 2005; 38:367-374. [PubMed: 15698843]

12. Arora P, Rajagopalam S, Ranjan R, Kolli H, Singh M, Venuto R, Lohr J. Preoperative Use of Angiotensin-Converting Enzyme Inhibitors/Angiotensin Receptor Blockers Is Associated with Increased Risk for Acute Kidney Injury after Cardiovascular Surgery. Clinical Journal of the American Society of Nephrology. 2008; 3:1266-1273. [PubMed: 18667735]

13. Arriero M, Brodsky SV, Gealekman O, Lucas PA, Goligorsky MS. Adult skeletal muscle stem cells differentiate into endothelial lineage and ameliorate renal dysfunction after acute ischemia. American Journal of Physiology - Renal Physiology. 2004; 287:F621-F627. [PubMed: 15198930]

14. Ashworth SL, Molitoris BA. Pathophysiology and functional significance of apical membrane disruption during ischemia. Current Opinion in Nephrology \& Hypertension. 1999; 8:449-458. [PubMed: 10491740]

15. Ashworth SL, Sandoval RM, Hosford M, Bamburg JR, Molitoris BA. Ischemic injury induces ADF relocalization to the apical domain of rat proximal tubule cells. American Journal of Physiology - Renal Fluid \& Electrolyte Physiology. 2001; 280:F886-F894.

16. Askenazi D, Feig D, Graham N, Hui-Stickle S, Goldstein SL. 3-5 year longitudinal follow-up of pediatric patients after acute renal failure. Kidney Int. 2006; 69:184-189. [PubMed: 16374442]

17. Ates E, Geno E, Erkasap N, Erkasap S, Akman S, Firat P, Emre Sk, Kiper H. Renal protection by brief liver ischemia in rats. Transplantation. 2002; 74:1247-1251. [PubMed: 12451261]

18. Atkinson SJ, Hosford MA, Molitoris BA. Mechanism of Actin Polymerization in Cellular ATP Depletion. Journal of Biological Chemistry. 2004; 279:5194-5199. [PubMed: 14623892]

19. Aufricht C, Lu E, Thulin G, Kashgarian M, Siegel NJ, Van Why SK. ATP releases HSP-72 from protein aggregates after renal ischemia. American Journal of Physiology. 1998; 274:F268-F274. [PubMed: 9486221]

20. Bacallao R, Fine LG. Molecular events in the organization of renal tubular epithelium: from nephrogenesis to regeneration. Am J Physiol. 1989; 257:F913-F924. [PubMed: 2690642]

21. Bacallao R, Garfinkel A, Monke S, Zampighi G, Mandel L. ATP depletion: a novel method to study junctional properties in epithelial tissues. I. Rearrangement of the actin cytoskeleton. J Cell Sci. 1994; 107:3301-3313. [PubMed: 7706387]

22. Badr KF, Ichikawa I. Prerenal failure: a deleterious shift from renal compensation to decompensation. N Engl J Med. 1988; 319:623-629. [PubMed: 3045546]

23. Bae EH, Lee J, Ma SK, Kim IJ, Frøkiaer, Nielsen Sr, Kim SY, Kim SW. a-Lipoic acid prevents cisplatin-induced acute kidney injury in rats. Nephrology Dialysis Transplantation. 2009; 24:2692-2700.

24. Bagnasco S, Good D, Balaban R, Burg M. Lactate production in isolated segments of the rat nephron. Am J Physiol. 1985; 248:F522-F526. [PubMed: 3985159]

25. Bagshaw SM, Laupland KB, Doig CJ, Mortis G, Fick GH, Mucenski M, Godinez-Luna T, Svenson LW, Rosenal T. Prognosis for long-term survival and renal recovery in critically ill patients with 
severe acute renal failure: a population-based study. Crit Care. 2005; 9:R700-R709. [PubMed: 16280066]

26. Baldwin DD, Maynes LJ, Berger KA, Desai PJ, Zuppan CW, Zimmerman GJ, Winkielman AM, Sterling TH, Tsai CK, Ruckle HC. Laparoscopic warm renal ischemia in the solitary porcine kidney model. Urology. 2004; 64:592-597. [PubMed: 15351615]

27. Baliga R, Zhang Z, Baliga M, Ueda N, Shah SV. In vitro and in vivo evidence suggesting a role for iron in cisplatin-induced nephrotoxicity. Kidney Int. 1998; 53:394-401. [PubMed: 9461098]

28. Bard JBL, McConnell JE, Davies JA. Towards a genetic basis for kidney development. Mechanisms of Development. 1994; 48:3-11. [PubMed: 7833287]

29. Basile DP. The endothelial cell in ischemic acute kidney injury: implications for acute and chronic function. Kidney Int. 2007; 72:151-156. [PubMed: 17495858]

30. Basile DP, Donohoe DL, Cao X, Van Why S. Resistance to ischemic acute renal failure in the Brown Norway rat: A new model to study cytoprotection. Kidney Int. 2004; 65:2201-2211. [PubMed: 15149333]

31. Basile DP, Donohoe DL, Roethe K, Mattson DL. Chronic renal hypoxia following ischemia/ reperfusion injury: Effects of L-Arginine on hypoxia and secondary damage. Am J Physiol Renal Physiol. 2003; 284:F338-F348. [PubMed: 12388385]

32. Basile DP, Donohoe DL, Roethe K, Osborn JL. Renal ischemic injury results in permanent damage to peritubular capillaries and influences long-term function. American Journal of Physiology. 2001; 281:F887-F899. [PubMed: 11592947]

33. Basile DP, Fredrich K, Chelladurai B, Leonard EC, Parrish AR. Renal ischemia reperfusion inhibits VEGF expression and induces ADAMTS-1, a novel VEGF inhibitor. Am J Physiol Renal Physiol. 2008; 294

34. Basile DP, Fredrich K, Weihrauch DW, Hattan N, Chilian WM. Angiostatin and matrix metalloprotease expression following ischemic acute renal failure. Am J Physiol Renal Physiol. 2004; 286:F893-F902. [PubMed: 15075185]

35. Basile DP, Friedrich JL, Spahic J, Knipe NL, Mang HE, Leonard EC, Ashtiyani SC, Bacallao RL, Molitoris BA, Sutton TA. Impaired endothelial proliferation and mesenchymal transition contribute to vascular rarefaction following acute kidney injury. American Journal of Physiology Renal Physiology. 2011; 300:F721-F733. [PubMed: 21123492]

36. Basile DP, Liapis H, Hammerman MR. Expression of bcl-2 and bax in regenerating rat renal tubules following ischemic injury. American Journal of Physiology - Renal Fluid \& Electrolyte Physiology. 1997; 41:F 640-F 647.

37. Basile DP, Martin DR, Hammerman MH. Extracellular matrix related genes in kidney postischemic injury: Potential role for TGF- $\beta$ in repair. American Journal of Physiology-Renal Physiology. 1998; 275 (Renal Physiol, 44):F894-F903.

38. Basile DP, Rovak JM, Martin DR, Hammerman MR. Increased transforming growth factor-s expression in regenerating rat renal tubules following ischemic injury. American Journal of Physiology. 1996; 270 (Renal Fluid Electrolyte Physiol. 39):F500-F509. [PubMed: 8780254]

39. Basnakian AG, Apostolov EO, Yin X, Napirei M, Mannherz HG, Shah SV. Cisplatin Nephrotoxicity Is Mediated by Deoxyribonuclease I. J Am Soc Nephrol. 2005; 16:697-702. [PubMed: 15647342]

40. Basnakian AG, Ueda N, Hong X, Galitovsky VE, Yin X, Shah SV. Ceramide synthase is essential for endonuclease-mediated death of renal tubular epithelial cells induced by hypoxiareoxygenation. Am J Physiol Renal Physiol. 2005; 288:F308-F314. [PubMed: 15479855]

41. Basnakian AG, Ueda N, Kaushal GP, Mikhailova MV, Shah SV. DNase I-Like Endonuclease in Rat Kidney Cortex That Is Activated during Ischemia/Reperfusion Injury. J Am Soc Nephrol. 2002; 13:1000-1007. [PubMed: 11912259]

42. Bastin J, Cambon N, Thompson M, Lowry OH, Burch HB. Change in energy reserves in different segments of the nephron during brief ischemia. Kidney Int. 1987; 31:1239-1247. [PubMed: 3613402]

43. Bayati A, Hellberg O, Odlind B, Wolgast M. Prevention of ischaemic acute renal failure with superoxide dismutase and sucrose. Acta Physiologica Scandinavica. 1987; 130:367-372. [PubMed: 3630718] 
44. Bayir H, Kagan V. Bench-to-bedside review: Mitochondrial injury, oxidative stress and apoptosis there is nothing more practical than a good theory. Critical Care. 2008; 12:206. [PubMed: 18341705]

45. Bellomo R, Chapman M, Hickling K, Myburgh J. Low-dose dopamine in patients with early renal dysfunction: a placebo-controlled randomised trial. The Lancet. 2000; 356:2139-2143.

46. Benjamin IJ, McMillan DR. Stress (heat shock) proteins: molecular chaperones in cardiovascular biology and disease. Circulation Research. 1998; 83:117-132. [PubMed: 9686751]

47. Bennett M, Dent CL, Ma Q, Dastrala S, Grenier F, Workman R, Syed H, Ali S, Barasch J, Devarajan P. Urine NGAL Predicts Severity of Acute Kidney Injury After Cardiac Surgery: A Prospective Study. Clinical Journal of the American Society of Nephrology. 2008; 3:665-673. [PubMed: 18337554]

48. Bernard GR, Vincent J-L, Laterre P-F, LaRosa SP, Dhainaut J-F, Lopez-Rodriguez A, Steingrub JS, Garber GE, Helterbrand JD, Ely EW, Fisher CJ. Efficacy and Safety of Recombinant Human Activated Protein C for Severe Sepsis. New England Journal of Medicine. 2001; 344:699-709. [PubMed: 11236773]

49. Bia M, Tyler K. Effect of cyclosporine on renal ischemic injury. Transplantation. 1987; 43:800804. [PubMed: 3590299]

50. Bidmon B, Endemann M, Muller T, Arbeiter K, Herkner K, Aufricht C. Heat shock protein-70 repairs proximal tubule structure after renal ischemia. Kidney Int. 2000; 58:2400-2407. [PubMed: 11115073]

51. Biju MP, Akai Y, Shrimanker N, Haase VH. Protection of HIF-1-deficient primary renal tubular epithelial cells from hypoxia-induced cell death is glucose dependent. American Journal of Physiology - Renal Physiology. 2005; 289:F1217-F1226. [PubMed: 16048903]

52. Bishop AL, Hall A. Rho GTPases and their effector proteins. Biochem J. 2000; 348:241-255. [PubMed: 10816416]

53. Blantz RC, Pelayo JC. A functional role for the tubuloglomerular feedback mechanism. Kidney Int. 1984; 25:739-746. [PubMed: 6381854]

54. Blazer-Yost BL, Watanabe M, Haverty TP, Ziyadeh FN. Role of insulin and IGF1 receptors in proliferation of cultured renal proximal tubule cells. Biochimica et Biophysica Acta. 1992; 1133:329-335. [PubMed: 1310625]

55. Blydt-Hansen TD, Katori M, Lassman C, Ke B, Coito AJ, Iyer S, Buelow R, Ettenger R, Busuttil RW, Kupiec-Weglinski JW. Gene Transfer-Induced Local Heme Oxygenase-1 Overexpression Protects Rat Kidney Transplants From Ischemia/Reperfusion Injury. Journal of the American Society of Nephrology. 2003; 14:745-754. [PubMed: 12595512]

56. Bohle A, Jahnecke J, Meyer D, Schubert GE. Morphology of acute renal failure: comparative data from biopsy and autopsy. Kidney Int Suppl. 1976; 6:S9-S16. [PubMed: 1068332]

57. Bolisetty S, Agarwal A. Neutrophils in acute kidney injury: not neutral any more. Kidney Int. 2009; 75:674-676. [PubMed: 19282858]

58. Bonomini V, Stefoni S, Vagelista A. Long-term patient and renal prognosis in acute renal failure. Nephron. 1984; 36:169-172. [PubMed: 6700808]

59. Bonventre JV. Kidney ischemic preconditioning. Current Opinion in Nephrology \& Hypertension. 2002; 11:43-48. [PubMed: 11753086]

60. Bonventre JV. Mechanisms of ischemic acute renal failure. Kidney Int. 1993; 43:1160-1178. [PubMed: 8510397]

61. Bonventre JV, Brezis M, Siegel NJ, Rosen S, Portilla D, Venkatachalam MA. Acute renal failure I: Relative importance of proximal vs. distal tubular injury. Am J Physiol. 1998:F623-F632.

62. Bonventre JV, Weinberg JM. Recent advances in the pathophysiology of ischemic acute renal failure. Journal of the American Society of Nephrology. 2003; 14:2199-2210. [PubMed: 12874476]

63. Bonventre JV, Zuk A. Ischemic acute renal failure: An inflammatory disease? Kidney Int. 2004; 66:480-485. [PubMed: 15253693]

64. Bor M, Durmuş O, Bilgihan A, Çevik C, Türközkan N. The beneficial effect of 2'deoxycoformycin in renal ischemia-reperfusion is mediated both by preservation of tissue ATP 
and inhibition of lipid peroxidation. International Journal of Clinical \& Laboratory Research. 1999; 29:75-79. [PubMed: 10436265]

65. Borkan SC, Gullans SR. MOLECULAR CHAPERONES IN THE KIDNEY. Annual Review of Physiology. 2002; 64:503-527.

66. Borthwick E, Ferguson A. Perioperative acute kidney injury: risk factors, recognition, management, and outcomes. BMJ. 341

67. Bosch X, Poch E, Grau JM. Rhabdomyolysis and Acute Kidney Injury. New England Journal of Medicine. 2009; 361:62-72. [PubMed: 19571284]

68. Brady, H.; Brenner, B.; Clarkson, M.; Lieberthal, W. Acute Renal Failure. In: Brenner, B., editor. The Kidney. Philadelphia: WB Saunders; 2000. p. 1201-1262.

69. Brady HR, Kone BC, Stromski ME, Zeidel ML, Giebisch G, Gullans SR. Mitochondrial injury: an early event in cisplatin toxicity to renal proximal tubules. Am J Physiol Renal Physiol. 1990; 258:F1181-F1187.

70. Brezis M, Rosen S. Hypoxia of the renal medulla-its implications for disease. New England Journal of Medicine. 1995; 332:647-657. [PubMed: 7845430]

71. Briggs J, Kennedy A, Young L, Luke R, Gray M. Renal function after acute tubular necrosis. British Med J. 1967; 3:513-516.

72. Bright, R. Reports of Medical Cases, Selected with a View of Illustrating the Symptoms and Cure of Diseases by a Reference to Morbid Anatomy. London: Richard Taylor for Longman, Rees, Orme, Browne, and Greene; 1827.

73. Brivet FG, Kleinknecht DJ, Loirat P, Landais PJ. Acute renal failure in intensive care units-causes, outcome, and prognostic factors of hospital mortality; a prospective, multicenter study. French Study Group on Acute Renal Failure. Crit Care Med. 1996; 24:192-198. [PubMed: 8605788]

74. Brodsky SV, Yamamoto T, Tada T, Kim B, Chen J, Kajiya F, Goligorsky MS. Endothelial dysfunction in ischemic acute renal failure: rescue by transplanted endothelial cells. American Journal of Physiology - Renal Fluid \& Electrolyte Physiology. 2002; 282:F1140-F1149.

75. Broekema M, Harmsen MC, van Luyn M, Koerts J, Persersen AH, Kooten TG, van Goor H, Navis G, Popa ER. Bone marrow-derived myofibroblasts contribute to renal interstital myofibroblasts population and produce procollagen I after ischemia reperfusion in rats. J Am Soc Nephrol. 2007 In press.

76. Brooks C, Wei Q, Cho S, Dong Z. Regulation of mitochondrial dynamics in acute kidney injury in cell culture and rodent models. Journal of Clinical Investigation. 2009; 119:1275-1285. [PubMed: 19349686]

77. Bulent Gul C, Gullulu M, Oral B, Aydinlar A, Oz O, Budak F, Yilmaz Y, Yurtkuran M. Urinary IL-18: A marker of contrast-induced nephropathy following percutaneous coronary intervention? Clinical Biochemistry. 2008; 41:544-547. [PubMed: 18237555]

78. Burne-Taney MJ, Rabb H. The role of adhesion molecules and T cells in ischemic renal injury. Current Opinion in Nephrology \& Hypertension. 2003; 12:85-90. [PubMed: 12496671]

79. Burne-Taney MJ, Yokota N, Rabb H. Persistent renal and extrarenal immune changes after severe ischemic injury. Kidney Int. 2005; 67:1002-1009. [PubMed: 15698438]

80. Bush KT, Tsukamoto T, Nigam SK. Selective degradation of E-cadherin and dissolution of Ecadherin-catenin complexes in epithelial ischemia. American Journal of Physiology - Renal Physiology. 2000; 278:F847-F852. [PubMed: 10807598]

81. Bussolati B, Bruno S, Grange C, Buttiglieri S, Deregibus MC, Cantino D, Camussi G. Isolation of renal progenitor cells from adult human kidney. American Journal of Pathology. 2005; 166:545555. [PubMed: 15681837]

82. Bussolati B, Tetta C, Camussi G. Contribution of Stem Cells to Kidney Repair. American Journal of Nephrology. 2008; 28:813-822. [PubMed: 18535367]

83. Buyukgebiz O, Aktan AÖ, Haklar G, Yalcin AS, Yeğen C, Yalin R, Ercan ZS. BQ-123, a specific endothelin (ET1) receptor antagonist, prevents ischemia-reperfusion injury in kidney transplantation. Transplant International. 1996; 9:201-207. [PubMed: 8723187]

84. Bywaters E, Beal D. Crush injuries and renal function. British Medical Journal. 1941; 1:427-432. [PubMed: 20783577] 
85. Castaneda MP, Swiatecka-Urban A, Mitsnefes MM, Feuerstein D, Kaskel FJ, Tellis V, Devarajan P. Activation of mitochondrial apoptotic pathways in human renal allografts after ischemiareperfusion injury. Transplantation. 2003; 76:50-54. [PubMed: 12865785]

86. Chandraker A, Takada M, Nadeau KC, Peach R, Tilney NL, Sayegh MH. CD28-b7 blockade in organ dysfunction secondary to cold ischemia/reperfusion injury. Kidney Int. 1997; 52:16781684. [PubMed: 9407517]

87. Chatterjee PK, Cuzzocrea S, Brown PA, Zacharowski K, Stewart KN, Mota-Filipe H, Thiemermann C. Tempol, a membrane-permeable radical scavenger, reduces oxidant stressmediated renal dysfunction and injury in the rat. Kidney International. 2000; 58:658-673. [PubMed: 10916089]

88. Chen C-F, Tsai S-Y, Ma M-C, Wu M-S. Hypoxic preconditioning enhances renal superoxide dismutase levels in rats. The Journal of Physiology. 2003; 552:561-569. [PubMed: 14561837]

89. Chen G, Bridenbaugh EA, Akintola AD, Catania JM, Vaidya VS, Bonventre JV, Dearman AC, Sampson HW, Zawieja DC, Burghardt RC, Parrish AR. Increased susceptibility of aging kidney to ischemic injury: identification of candidate genes changed during aging, but corrected by caloric restriction. American Journal of Physiology - Renal Physiology. 2007; 293:F1272-F1281. [PubMed: 17670906]

90. Chen J, John R, Richardson JA, Shelton JM, Zhou XJ, Wang Y, Wu QQ, Hartono JR, Winterberg $\mathrm{PD}, \mathrm{Lu} \mathrm{CY}$. Toll-like receptor 4 regulates early endothelial activation during ischemic acute kidney injury. Kidney Int. 2010

91. Chen Y-F, Li P-L, Zou A-P. Oxidative stress enhances the production and actions of adenosine in the kidney. American Journal of Physiology - Regulatory, Integrative and Comparative Physiology. 2001; 281:R1808-R1816.

92. Chen Z, Chua CC, Ho Y-S, Hamdy RC, Chua BHL. Overexpression of Bcl-2 attenuates apoptosis and protects against myocardial I/R injury in transgenic mice. Am J Physiol Heart Circ Physiol. 2001; 280:H2313-H2320. [PubMed: 11299236]

93. Chertow GM, Burdick E, Honour M, Bonventre JV, Bates DW. Acute Kidney Injury, Mortality, Length of Stay, and Costs in Hospitalized Patients. Journal of the American Society of Nephrology. 2005; 16:3365-3370. [PubMed: 16177006]

94. Chertow GM, Christiansen CL, Cleary PD, Munro C, Lazarus JM. Prognostic stratification in critically ill patients with acute renal failure requiring dialysis. Arch Intern Med. 1995; 155:15051511. [PubMed: 7605152]

95. Choudhury D, Ahmed Z. Drug-associated renal dysfunction and injury. Nat Clin Pract Nephrol. 2006; 2:80-91. [PubMed: 16932399]

96. Coca SG, Yalavarthy R, Concato J, Parikh CR. Biomarkers for the diagnosis and risk stratification of acute kidney injury: a systematic review. Kidney Int. 2008; 73:1008-1016. [PubMed: 18094679]

97. Coca SG, Yusuf B, Shlipak MG, Garg AX, Parikh CR. Long-term Risk of Mortality and Other Adverse Outcomes After Acute Kidney Injury: A Systematic Review and Meta-analysis. American Journal of Kidney Diseases. 2009; 53:961-973. [PubMed: 19346042]

98. Conesa, ELp; Valero, F.; Nadal, JC.; Fenoy, FJ.; López, B.; Arregui, Ba; Salom, MGa. N-acetyl-1cysteine improves renal medullary hypoperfusion in acute renal failure. American Journal of Physiology - Regulatory, Integrative and Comparative Physiology. 2001; 281:R730-R737.

99. Conger JD, Robinette JB, Guggenheim SJ. Effect of acetylcholine on the early phase of reversible norepinephrine-induced acute renal failure. Kidney Int. 1981; 19:399-409. [PubMed: 7241880]

100. Conger JD, Robinette JB, Hammond WS. Differences in vascular reactivity in models of ischemic acute renal failure. Kidney Int. 1991; 39:1087-1097. [PubMed: 1895663]

101. Conger JD, Robinette JB, Schrier RW. Smooth muscle calcium and endothelium derived relaxing factor in the abnormal vascular responses of acute renal failure. Journal of Clinical Investigation. 1988; 82:532-537. [PubMed: 3261301]

102. Conger JD, Weil JV. Abnormal vascular function following ischemiareperfusion injury. Journal of Investigative Medicine. 1995; 43:431-442. [PubMed: 8528754] 
103. Coopersmith CM, O'Donnell D, Gordon JI. Bcl-2 inhibits ischemia-reperfusioninduced apoptosis in the intestinal epithelium of transgenic mice. Am J Physiol Gastrointest Liver Physiol. 1999; 276:G677-G686.

104. Cowley AW Jr. Role of the renal medulla in volume and arterial pressure regulation. 1997 Jul.:R1-R15.

105. Crompton M. The mitochondrial permeability transition pore and its role in cell death. Biochem J. 1999; 341:233-249. [PubMed: 10393078]

106. Cruthirds DL, Novak L, Akhi KM, Sanders PW, Thompson JA, MacMillan-Crow LA. Mitochondrial targets of oxidative stress during renal ischemia/reperfusion. Archives of Biochemistry \& Biophysics. 2003; 412:27-33. [PubMed: 12646264]

107. Cunningham PN, Dyanov HM, Park P, Wang J, Newell KA, Quigg RJ. Acute Renal Failure in Endotoxemia Is Caused by TNF Acting Directly on TNF Receptor-1 in Kidney. J Immunol. 2002; 168:5817-5823. [PubMed: 12023385]

108. Cunningham PN, Wang Y, Guo R, He G, Quigg RJ. Role of Toll-Like Receptor 4 in EndotoxinInduced Acute Renal Failure. The Journal of Immunology. 2004; 172:2629-2635. [PubMed: 14764737]

109. Cuppage FE, Chiga M, Tate A. Cell cycle studeis in the regenerating rat nephron following injury with mercuric chloride. Laboratory Investigation. 1972; 26:122-126. [PubMed: 5009212]

110. Cuppage FE, Cunningham N, Tate A. Nucleic acid synthesis in the regenerating nephron following injury with mercuric chloride. Laboratory Investigation. 1969; 21:449-457. [PubMed: 5351490]

111. Cuttle L, Zhang X-J, Endre ZH, Winterford C, Gobe GC. Bcl-XL translocation in renal tubular epithelial cells in vitro protects distal cells from oxidative stress. Kidney Int. 2001; 59:17791788. [PubMed: 11318948]

112. Dagher F, Pollina RM, Rogers DM, Gennaro M, Ascer E. The value and limitations of L-arginine infusion on glomerular and tubular function in the ischemic/reperfused kidney. Journal of Vascular Surgery. 1995; 21:453-458. [PubMed: 7877227]

113. Dagher PC, Basile DP. An expanding role of Toll-like receptors in sepsisinduced acute kidney injury. American Journal of Physiology - Renal Physiology. 2008; 294:F1048-F1049. [PubMed: 18353874]

114. Dagher PC, Herget-Rosenthal S, Ruehm SG, Jo S-K, Star RA, Agarwal R, Molitoris BA. Newly developed techniques to study and diagnose acute renal failure. Journal of the American Society of Nephrology. 2003; 14:2188-2198. [PubMed: 12874475]

115. Dagher Pierre C. Apoptosis in ischemic renal injury: Roles of GTP depletion and p53. Kidney Int. 2004; 66:506-509. [PubMed: 15253698]

116. Dawidson I, Rooth P. Effects of calcium antagonists in ameliorating cyciosporine A nephrotoxicity and post-transplant ATN. 1990:233-246.

117. Day Y-J, Huang L, Ye H, Li L, Linden J, Okusa MD. Renal Ischemia-Reperfusion Injury and Adenosine 2A Receptor-Mediated Tissue Protection: The Role of CD4+ T Cells and IFN- $\gamma$. The Journal of Immunology. 2006; 176:3108-3114. [PubMed: 16493070]

118. De Greef KE, Ysebaert DK, Dauwe S, Persy V, Vercauteren SR, Mey D, De Broe ME. Anti-B7-1 blocks mononuclear cell adherence in vasa recta after ischemia. Kidney International. 2001; 60:1415-1427. [PubMed: 11576355]

119. de la Coste A, Fabre M, McDonell N, Porteu A, Gilgenkrantz H, Perret C, Kahn A, Mignon A. Differential protective effects of Bcl-xL and Bcl-2 on apoptotic liver injury in transgenic mice. Am J Physiol Gastrointest Liver Physiol. 1999; 277:G702-G708.

120. Dear JW, Yasuda H, Hu X, Hieny S, Yuen PST, Hewitt SM, Sher A, Star RA. Sepsis-induced organ failure is mediated by different pathways in the kidney and liver: Acute renal failure is dependent on MyD88 but not renal cell apoptosis. Kidney Int. 2006; 69:832-836. [PubMed: 16518342]

121. Denton Mark D, Chertow GM, Brady HR. [ldquo]Renal-dose[rdquo] dopamine for the treatment of acute renal failure: Scientific rationale, experimental studies and clinical trials. Kidney Int. 1996; 50:4-14. [PubMed: 8807566] 
122. Devalaraja-Narashimha K, Diener AM, Padanilam BJ. Cyclophilin D gene ablation protects mice from ischemic renal injury. American Journal of Physiology - Renal Physiology. 2009; 297:F749-F759. [PubMed: 19553348]

123. Devarajan P, Mishra J, Supavekin S, Patterson LT, Steven Potter S. Gene expression in early ischemic renal injury: clues towards pathogenesis, biomarker discovery, and novel therapeutics. Molecular Genetics and Metabolism. 2003; 80:365-376. [PubMed: 14654349]

124. di Mari JF, Davis R, Safirstein RL. MAPK activation determines renal epithelial cell survival during oxidative injury. American Journal of Physiology. 1999; 277:F195-F203. [PubMed: 10444573]

125. DiMari J, Megyesi J, Udvarhelyi N, Price P, Davis R, Safirstein R. N-acetyl cysteine ameliorates ischemic renal failure. American Journal of Physiology - Renal Physiology. 1997; 272:F292_ F298.

126. Djamali A, Sadowski E, Muehrer RJ, reese S, Smavatkul C, Vidyasagar A, Fain SB, Lipscomb RC, Hullett DH, Samaniego-Picota M, Grist TM, Becker BN. BOLD MRI assessment of intrarenal oxygenation and oxidative stress in patients with chronic kidney alograft dysfunction. Am J Physiol. 2007; 292:F513-F522.

127. Dobashi K, Ghosh B, Orak JK, Singh I, Singh AK. Kidney ischemiareperfusion: Modulation of antioxidant defenses. Molecular and Cellular Biochemistry. 2000; 205:1-11. [PubMed: 10821417]

128. Doctor R, Mandel L. Minimal role of xanthine oxidase and oxygen free radicals in rat renal tubular reoxygenation injury. Journal of the American Society of Nephrology. 1991; 1:959-969. [PubMed: 1883966]

129. Dong X, Swaminathan S, Bachman LA, Croatt AJ, Nath KA, Griffin MD. Resident dendritic cells are the predominant TNF-secreting cell in early renal ischemiareperfusion injury. Kidney Int. 2007; 71:619-628. [PubMed: 17311071]

130. Dong Z. Response to / Autophagy: a protective mechanism against nephrotoxicant-induced renal injury/'. Kidney Int. 75:119-119.

131. Donnahoo KK, Meng X, Ao L, Ayala A, Shames BD, Cain MP, Harken AH, Meldrum DR. Differential cellular immunolocalization of renal tumour necrosis factor-a production during ischaemia versus endotoxaemia. Immunology. 2001; 102:53-58. [PubMed: 11168637]

132. Donnahoo KK, Meng X, Ayala A, Cain MP, Harken AH, Meldrum DR. Early kidney TNF--alpha expression mediates neutrophil infiltration and injury after renal ischemia-reperfusion. American Journal of Physiology - Regulatory, Integrative and Comparative Physiology. 1999; 277:R922R929.

133. Donohoe JF, Venkatchalam MA, Bernard DB, Levinsky NG. Tubular leakage and obstruction after renal ischemia: Structural-functional correlations. Kidney Int. 1978; 13:208-222. [PubMed: 651122]

134. Du C, Guan Q, Diao H, Yin Z, Jevnikar AM. Nitric oxide induces apoptosis in renal tubular epithelial cells through activation of caspase-8. Am J Physiol Renal Physiol. 2006; 290:F1044F1054. [PubMed: 16352744]

135. Du C, Guan Q, Yin Z, Zhong R, Jevnikar AM. IL-2-mediated apoptosis of kidney tubular epithelial cells is regulated by the caspase-8 inhibitor c-FLIP. Kidney Int. 2005; 67:1397-1409. [PubMed: 15780092]

136. Duffield JS. Macrophages and Immunologic Inflammation of the Kidney. Seminars in Nephrology. 2010; 30:234-254. [PubMed: 20620669]

137. Dunnill MS. A review of the pathology and pathogenesis of acute renal failure due to acute tubular necrosis. J Clin Pathol. 1974; 27:2-13. [PubMed: 16811078]

138. Edelstein CL. Biomarkers of Acute Kidney Injury. Advances in chronic kidney disease. 2008; 15:222-234. [PubMed: 18565474]

139. Eickelberg O, Seebach FA, Riordan M, Thulin G, Mann A, Reidy K, Van Why SK, Kashgarian M, Siegel NJ. Functional activation of heat shock factor and hypoxiainducible factor in the kidney. J Am Soc Nephrol. 2002; 13:2094-2101. [PubMed: 12138141]

140. Emami A, Schwartz JH, Borkan SC. Transient ischemia or heat stress induces a cytoprotectant protein in rat kidney. Am J Physiol. 1991; 260:F479-F485. [PubMed: 2012203] 
141. Enestrom S, Druid H, Rammer L. Fibrin deposition in the kidney in postischaemic renal damage. British Journal of Experimental Pathology. 1988; 69:387-394. [PubMed: 3291926]

142. Erley CM, Duda SH, Schlepckow S, Koehler J, Huppert PE, Strohmaier WL, Bohle A, Risler T, Osswald H. Adenosine antagonist theophylline prevents the reduction of glomerular filtration rate after contrast media application. Kidney Int. 1994; 45:1425-1431. [PubMed: 8072255]

143. Faubel S, Ljubanovic D, Reznikov L, Somerset H, Dinarello CA, Edelstein CL. Caspase-1deficient mice are protected against cisplatin-induced apoptosis and acute tubular necrosis. Kidney Int. 2004; 66:2202-2213. [PubMed: 15569309]

144. Feldkamp T, Kribben A, Weinber JM. Assessment of mitochondrial membrane potential in proximal tubules after hypoxia-reoxygenation injury. Am J Physiol Renal Physiol. 2005; 288:F1092-F1102. [PubMed: 15625081]

145. Feldkamp T, Park JS, Pasupulati R, Amora D, Roeser NF, Venkatachalam MA, Weinberg JM. Regulation of the mitochondrial permeability transition in kidney proximal tubules and its alteration during hypoxia-reoxygenation. Am J Physiol Renal Physiol. 2009; 297:F1632-F1646. [PubMed: 19741014]

146. Fernando AR, Armstrong DMG, Griffiths JR, Hendry WF, O'Donoghue EPN, Perrett D, Ward JP, Wickham JEA. INOSINE IN EXPERIMENTAL ACUTE RENAL FAILURE. The Lancet. 1976; 308:1302-1303.

147. Finn, WF. Recovery from acute renal failure. In: Lazarus, JM.; B B., editors. Acute Renal Failure. New York: Churchill Livingstone; 1993. p. 553-596.

148. Finn WF, Hak LJ, Grossman SH. Protective effect of prostacyclin on postischemic acute renal failure in the rat. Kidney Int. 1987; 32:479-487. [PubMed: 3323594]

149. Firth JD, Raine AEG, Ratcliffe PJ, Ledingham JGG. ENDOTHELIN: AN IMPORTANT FACTOR IN ACUTE RENAL FAILURE? The Lancet. 1988; 332:1179-1182.

150. Fissell WH, Dyke DB, Weitzel WF, Buffington DA, Westover AJ, MacKay SM, Gutierrez JM, Humes HD. Bioartificial kidney alters cytokine response and hemodynamics in endotoxinchallenged uremic animals. Blood Purification. 2002; 20(1):55-60. 2002. [PubMed: 11803160]

151. Floege J, Burns MW, Alpers CE, Yoshimura A, Pritzl P, Gordon K, Seifert RA, Bowen-Pope DF, Couser WG, Johnson RJ. Glomerular cell proliferation and PDGF expression precede glomerulosclerosis in the remnant kidney model. Kidney Int. 1992; 41:297-309. [PubMed: 1313122]

152. Flores J, DiBona DR, Beck CH, Leaf A. The Role of Cell Swelling in Ischemic Renal Damage and the Protective Effect of Hypertonic Solute. The Journal of Clinical Investigation. 1972; 51:118-126. [PubMed: 5007042]

153. Forbes JM, Hewitson TD, Becker GJ, Jones CL. Simultaneous blockade of endothelin A and B receptors in ischemic acute renal failure is detrimental to long-term kidney function. Kidney International. 2001; 59:1333-1341. [PubMed: 11260394]

154. Fowler B. Mechanisms of kidney cell injury from metals. Environmental Health Perspect. 1993; 100:57-63.

155. Friedewald JJ, Rabb H. Inflammatory cells in ischemic acute renal failure. Kidney Int. 2004; 66:486-491. [PubMed: 15253694]

156. Fujii T, Kurata H, Takaoka M, Muraoka T, Fujisawa Y, Shokoji T, Nishiyama A, Abe Y, Matsumura Y. The role of renal sympathetic nervous system in the pathogenesis of ischemic acute renal failure. European Journal of Pharmacology. 2003; 481:241-248. [PubMed: 14642792]

157. Fuller TF, Rose F, Singleton KD, Linde Y, Hoff U, Freise CE, Dragun D, Niemann CU. Glutamine Donor Pretreatment in Rat Kidney Transplants with Severe Preservation Reperfusion Injury. The Journal of surgical research. 2007; 140:77-83. [PubMed: 17292409]

158. Fuller TF, Sattler B, Binder L, Vetterlein F, Ringe B, Lorf T. Reduction of severe ischemia/ reperfusion injury in rat idney grafts by a soluble P-Selectin glycprotein ligand. Tranplantation. 2001; 72:216-222.

159. Funk JA, Odejinmi S, Schnellmann RG. SRT1720 Induces Mitochondrial Biogenesis and Rescues Mitochondrial Function after Oxidant Injury in Renal Proximal Tubule Cells. Journal of Pharmacology and Experimental Therapeutics. 333:593-601. [PubMed: 20103585] 
160. Furuichi K, Wada T, Iwata Y, Sakai N, Yoshimoto K, Kobayashi K, Mukaida N, Matsushima K, Yokoyama H. Administration of FR167653, a new anti-inflammatory compound, prevents renal ischaemia/reperfusion injury in mice. Nephrology Dialysis Transplantation. 2002; 17:399-407.

161. Garcia-Criado FJ, Eleno N, Santos-Benito F, Valdunciel JJ, Reverte M, Lozano-Sanchez FS, Ludena MD, Gomez-Alonso A, Lopez-Novoa JM. Protective effect of exogenous nitric oxide on the renal function and inflammatory response in a model of ischemia-reperfusion.

Transplantation. 1998; 66:982-990. [PubMed: 9808479]

162. Gaudio KM, Ardito TA, Reilly HF, Kashgarian M, Siegel NJ. Accelerated cellular recovery after an ischemic renal injury. American Journal of Pathology. 1983; 112:338-346. [PubMed: 6604459]

163. Gaudio KM, Taylor MR, Chaudry IH, Kashgarian M, Siegel NJ. Accelerated recovery of single nephron function by the postischemic infusion of ATP-MgCl2. Kidney Int. 1982; 22:13-20. [PubMed: 7120752]

164. Gaudio KM, Thulin G, Ardito T, Kashgarian M, Siegel NJ. Redistribution of cellular energy following renal ischemia. Pediatric Nephrology. 1991; 5:591-596. [PubMed: 1911145]

165. Gellai M, Jugus M, Fletcher T, DeWolf R, Nambi P. Reversal of postischemic acute renal failure with a selective endothelinA receptor antagonist in the rat. The Journal of Clinical Investigation. 1994; 93:900-906. [PubMed: 8113422]

166. Genescà M, Sola A, Hotter G. Actin cytoskeleton derangement induces apoptosis in renal ischemia/reperfusion. Apoptosis. 2006; 11:563-571. [PubMed: 16528472]

167. Geng H, Lan R, Wang G, Siddiqi AR, Naski MC, Brooks AI, Barnes JL, Saikumar P, Weinberg JM, Venkatachalam MA. Inhibition of Autoregulated TGF-B Signaling Simultaneously Enhances Proliferation and Differentiation of Kidney Epithelium and Promotes Repair Following Renal Ischemia. The American journal of pathology. 2009; 174:1291-1308. [PubMed: 19342372]

168. Ghielli M, Verstrepen W, Nouwen E, De Broe ME. Regeneration processes in the kidney after acute injury: role of infiltrating cells. Exp Nephrol. 1998; 6:502-507. [PubMed: 9807021]

169. Glaumann B, Glaumann H, Berezesky I, Trump BF. Studies on the cellular recovery from injury. II. Ultrastructural studies on the recovery of the pars convoluta of the proximal tubule of the rate kidney from temporary ischemia. Virchows Arch B Cell Pathol. 1977; 24:1-18. [PubMed: 405790]

170. Glaumann B, Glaumann H, Berezesky I, Trump BF. Studies on the pathogenesis of ischemic cell injury. II. Morphological changes of the pars convolutia (P1 and P2) of the proximal tubule of the rat kidney made ischemic in vivo. Virchows Arch B Cell Pathol. 1975; 19:281-302. [PubMed: 813377]

171. Glaumann B, Trump BF. Studies on the pathogenesis of ischemic cell injury. III. Morphological changes of the proximal pars recta tubules (P3) of the rat kidney made ischemic in vivo. Virchows Arch B Cell Pathol. 1975; 19:303-332. [PubMed: 813378]

172. Gobe G, Zhang XJ, Cuttle L, Pat B, Willgoss D, Hancock J, Barnard R, Endre RB. Bcl-2 genes and growth factors in the pathology of ischaemic acute renal failure. Immunology \& Cell Biology. 1999; 77:279-286. [PubMed: 10361261]

173. Goligorsky MS, Lieberthal W, Racusen L, Simon EE. Integrin receptors in renal tubular epithelium: new insights into pathophysiology of acute renal failure. American Journal of Physiology. 1993; 264:F1-F8. [PubMed: 8430820]

174. Gomes M, Cancherini V, Reboucas MA, Reboucas NA. Ischemic Preconditioning of Renal Tissue: Identification of Early Up-Regulated Genes. Nephron Experimental Nephrology. 2003; 93:e107-e116. [PubMed: 12660413]

175. Gonzalez-Flecha B, Boveris A. Mitochondrial sites of hydrogen peroxide production in reperfused rat kidney cortex. Biochimica et Biophysica Acta (BBA) - General Subjects. 1995; 1243:361-366.

176. Gopalakrishnan S, Hallett MA, Atkinson SJ, Marrs JA. Differential regulation of junctional complex assembly in renal epithelial cell lines. American Journal of Physiology - Cell Physiology. 2003; 285:C102-C111. [PubMed: 12777255]

177. Grams ME, Rabb H. The distant organ effects of acute kidney injury. Kidney Int. 2011 
178. Gunter TE, Pfeiffer DR. Mechanisms by which mitochondria transport calcium. Am J Physiol Cell Physiol. 1990; 258:C755-C786.

179. Guo R, Wang Y, Minto AW, Quigg RJ, Cunningham PN. Acute Renal Failure in Endotoxemia is Dependent on Caspase Activation. J Am Soc Nephrol. 2004; 15:3093-3102. [PubMed: 15579512]

180. Guo S, Wharton W, Moseley P, Shi H. Heat shock protein 70 regulates cellular redox status by modulating glutathione-related enzyme activities. Cell Stress and Chaperones. 2007; 12:245-254. [PubMed: 17915557]

181. Gupta S, Verfaillie C, Chmielewski D, Kim Y, Rosenberg ME. A role for extrarenal cells in the regeneration following acute renal failure. Kidney Int. 2002; 62:1285-1290. [PubMed: 12234298]

182. Gupta S, Verfaillie C, Chmielewski D, Kren S, Eidman K, Connaire J, Heremans Y, Lund T, Blackstad M, Jiang Y, Luttun A, Rosenberg ME. Isolation and Characterization of KidneyDerived Stem Cells. Journal of the American Society of Nephrology. 2006; 17:3028-3040. [PubMed: 16988061]

183. Haase VH. Hypoxia-inducible factors in the kidney. American Journal of Physiology - Renal Physiology. 2006; 291:F271-F281. [PubMed: 16554418]

184. Hagen TM, Aw TY, Jones DP. Glutathione uptake and protection against oxidative injury in isolated kidney cells. Kidney Int. 1988; 34:74-81. [PubMed: 3172638]

185. Hall AM, Unwin RJ, Parker N, Duchen MR. Multiphoton Imaging Reveals Differences in Mitochondrial Function between Nephron Segments. J Am Soc Nephrol. 2009; 20:1293-1302. [PubMed: 19470684]

186. Hallett MA, Dagher PC, Atkinson SJ. Rho GTPases show differential sensitivity to nucleotide triphosphate depletion in a model of ischemic cell injury. American Journal of Physiology - Cell Physiology. 2003; 285:C129-C138. [PubMed: 12620811]

187. Hamerski DA, Santoro SA. Integrins and the kidney: biology and pathobiology. Current Opinion in Nephrology and Hypertension. 1999; 8:9-14. [PubMed: 9914855]

188. Hammerman MR, Rogers SA, Ryan G. Growth factors and metanephrogenesis. American Journal of Physiology. 1992; 262:F523-F532. [PubMed: 1566866]

189. Hammerschmidt E, Loeffler I, Wolf G. Morg1 heterozygous mice are protected from acute renal ischemia-reperfusion injury. American Journal of Physiology - Renal Physiology. 2009; 297:F1273-F1287. [PubMed: 19726548]

190. Haq M, Norman J, Saba S, Ramirez G, Rabb H. Role of IL-1 in renal ischemic reperfusion injury. Journal of the American Society of Nephrology. 1998; 9:614-619. [PubMed: 9555664]

191. Harris RC. Growth factors and cytokines in acute renal failure. Advances in Renal Replacement Therapy. 1997; 4:43-53. [PubMed: 9113240]

192. Harris RC, Breyer MD. Physiological regulation of cyclooxygenase-2 in the kidney. American Journal of Physiology - Renal Physiology. 2001; 281:F1-F11. [PubMed: 11399641]

193. Hasegawa K, Wakino S, Yoshioka K, Tatematsu S, Hara Y, Minakuchi H, Sueyasu K, Washida N, Tokuyama H, Tzukerman M, Skorecki K, Hayashi K, Itoh H. Kidney-specific Overexpression of Sirt1 Protects against Acute Kidney Injury by Retaining Peroxisome Function. Journal of Biological Chemistry. 285:13045-13056. [PubMed: 20139070]

194. Hashimoto-Uoshima M, Yan Y, Schneider G, Aukhil I. The alternatively spliced domains EIIIB and EIIIA of human fibronectin affect cell adhesion and spreading. J Cell Sci. 1997; 110:22712280. [PubMed: 9378776]

195. Haug CE, Colvin RB, Delmonico FL, Auchincloss HJ, Tolkoff-Rubin N, Preffer FI, Rothlein R, Norris S, Scharschmidt L, Cosimi AB. A PHASE I TRIAL OF IMMUNOSUPPRESSION WITH ANTIIC AM-1 (CD54) mAb IN RENAL ALLOGRAFT RECIPIENTS. Transplantation. 1993; 55:766-772. [PubMed: 8097341]

196. Hausenloy DJ, Yellon DM. Remote ischaemic preconditioning: underlying mechanisms and clinical application. Cardiovascular Research.

197. Havasi A, Li Z, Wang Z, Martin JL, Botla V, Ruchalski K, Schwartz JH, Borkan SC. Hsp27 Inhibits Bax Activation and Apoptosis via a Phosphatidylinositol 3-Kinasedependent Mechanism. Journal of Biological Chemistry. 2008; 283:12305-12313. [PubMed: 18299320] 
198. Havasi A, Wang Z, Gall JM, Spaderna M, Suri V, Canlas E, Martin JL, Schwartz JH, Borkan SC. Hsp27 inhibits sublethal, Src-mediated renal epithelial cell injury. American Journal of Physiology - Renal Physiology. 2009; 297:F760-F768. [PubMed: 19553351]

199. Healy E, Dempsey M, Lally C, Ryan MP. Apoptosis and necrosis: Mechanisms of cell death induced by cyclosporine A in a renal proximal tubular cell line. Kidney Int. 1998; 54:1955-1966. [PubMed: 9853260]

200. Hegarty NJ, Young LS, Kirwan CN, O'Neill AJ, Bouchier-Hayes DM, Sweeney P, Watson RWG, Fitzpatrick JM. Nitric oxide in unilateral ureteral obstruction: Effect on regional renal blood flow. Kidney Int. 2001; 59:1059-1065. [PubMed: 11231361]

201. Hellberg PO, Kallskog O, Wolgast M. Nephron function in the early phase of ischemic renal failure. Significance of erythrocyte trapping. Kidney Int. 1990; 38:432-439. [PubMed: 2232485]

202. Hellberg POA, Kallskog O, Wolgast M. Red cell trapping and postischemic renal blood flow. Differences between the cortex, outer and inner medulla. Kidney Int. 1991; 40:625-631. [PubMed: 1745011]

203. Hellberg POA, Kallskog OT, Ojteg G, Wolgast M. Peritubular capillary permeability and intravascular RBC aggregation after ischemia: effects of neutrophils. American Journal of Physiology. 1990; 258 (Renal Fluid Electrolyte Physiology):F1018-F1025. [PubMed: 2330969]

204. Herrera MB, Bussolati B, Bruno S, Morando L, Mauriello-Romanazzi G, Sanavio F, Stamenkovic I, Biancone L, Camussi G. Exogenous mesenchymal stem cells localize to the kidney by means of CD44 following acute tubular injury. Kidney Int. 2007; 72:430-441. [PubMed: 17507906]

205. Hill P, Shukla D, Tran MGB, Aragones J, Cook HT, Carmeliet P, Maxwell PH. Inhibition of Hypoxia Inducible Factor Hydroxylases Protects Against Renal Ischemia-Reperfusion Injury. Journal of the American Society of Nephrology. 2008; 19:39-46. [PubMed: 18178798]

206. Himmelfarb J, Joannidis M, Molitoris B, Schietz M, Okusa MD, Warnock D, Laghi F, Goldstein SL, Prielipp R, Parikh CR, Pannu N, Lobo SM, Shah S, D'Intini V, Kellum JA. Evaluation and initial management of acute kidney injury. Clin J Am Soc Nephrol. 2008; 3:962-967. [PubMed: 18354074]

207. Hirschberg R. Effects of growth hormone and IGF-I on glomerular ultrafiltration in growth hormone-deficient rats. Regulatory Peptides. 1993; 48:241-250. [PubMed: 8265813]

208. Hirschberg R, Kopple J, Lipsett P, Benjamin E, Minei J, Albertson T, Munger M, Metzler M, Zaloga G, Murray M, Lowry S, Conger J, McKeown W, O'Shea M, Baughman R, Wood K, Haupt M, Kaiser R, Simms H, Warnock D, Summer W, Hintz R, Myers B, Haenftling K, Capra W, Pike M, Guler H-P. Multicenter clinical trial of recombinant human insulinlike growth factor I in patients with acute renal failure. Kidney Int. 1999; 55:2423-2432. [PubMed: 10354291]

209. Hohenstein B, Kuo M-C, Addabbo F, Yasuda K, Ratliff B, Schwarzenberger C, Eckardt K-U, Hugo CPM, Goligorsky MS. Enhanced progenitor cell recruitment and endothelial repair after selective endothelial injury of the mouse kidney. American Journal of Physiology - Renal Physiology. 2010; 298:F1504-F1514. [PubMed: 20237239]

210. Hollenberg NK, Epstein FH, Rosen S, Basch R, Oken D, Merrill. Acute oliguric renal failure in man: evidence for preferential renal cortical ischemia. Medicne (Balt). 1968; 47:455-474.

211. Homma-Takeda S, Takenaka Y, Kumagai Y, Shimojo N. Selective induction of apoptosis of renal proximal tubular cells caused by inorganic mercury in vivo. Environmental Toxicology and Pharmacology. 1999; 7:179-187. [PubMed: 21781924]

212. Horbelt M, Lee S-Y, Mang HE, Knipe NL, Sado Y, Kribben A, Sutton TA. Acute and chronic microvascular alterations in a mouse model of ischemic acute kidney injury. Am J Physiol Renal Physiol. 2007; 293:F688-F695. [PubMed: 17626153]

213. Hou SH, Bushinsky DA, Wish JB, Cohen JJ, Harrington JT. Hospital-acquired renal insufficiency: a prospective study. Am J Med. 1983; 74:243-248. [PubMed: 6824004]

214. Hsu CY, McCulloch CE, Fan D, Ordonez JD, Chertow GM, Go AS. Communitybased incidence of acute renal failure. Kidney Int. 2007; 72:208-212. [PubMed: 17507907]

215. Huang C, Huang C, Hestin D, Dent PC, Barclay P, Collis M, Johns EJ. The effect of endothelin antagonists on renal ischaemia-reperfusion injury and the development of acute renal failure in the rat. Nephrology Dialysis Transplantation. 2002; 17:1578-1585. 
216. Huang H, He Z, Roberts LJ, Salahudeen AK. Deferoxamine Reduces Cold-Ischemic Renal Injury in a Syngeneic Kidney Transplant Model. American Journal of Transplantation. 2003; 3:15311537. [PubMed: 14629283]

217. Humes D, Cieslinski DA, Coimbra TM, Messana JM, Galvao C. Epidermal growth factor enhaces renal tubule cell regeneration and repair and accelerates the recovery of renal function in postischemic acute renal failure. The Journal of Clinical Investigation. 1989; 84:1757-1761. [PubMed: 2592559]

218. Humphreys BD, Lin S-L, Kobayashi A, Hudson TE, Nowlin BT, Bonventre JV, Valerius MT, McMahon AP, Duffield JS. Fate Tracing Reveals the Pericyte and Not Epithelial Origin of Myofibroblasts in Kidney Fibrosis. The American journal of pathology. 176:85-97. [PubMed: 20008127]

219. Humphreys BD, Valerius MT, Kobayashi A, Mugford JW, Soeung S, Duffield JS, McMahon AP, Bonventre JV. Intrinsic Epithelial Cells Repair the Kidney after Injury. Cell stem cell. 2008; 2:284-291. [PubMed: 18371453]

220. Hynninen MS, Niemi TT, Poyhia R, Raininko EI, Salmenperä MT, Lepantalo MJ, Railo MJ, Tallgren MK. N-Acetylcysteine for the Prevention of Kidney Injury in Abdominal Aortic Surgery: A Randomized, Double-Blind, Placebo-Controlled Trial. Anesthesia \& Analgesia. 2006; 102:1638-1645. [PubMed: 16717300]

221. Ichikawa I, Kiyama S, Yoshioka T. Renal antioxidant enzymes: Their regulation and function. Kidney Int. 1994; 45:1-9. [PubMed: 8126996]

222. Ichikawa I, Purkerson ML, Yates J, Klahr S. Dietary protein intake conditions the degree of renal vasoconstriction in acute renal failure caused by ureteral obstruction. American Journal of Physiology - Renal Physiology. 1985; 249:F54-F61.

223. Igawa T, Matsumoto K, Kanda S, Saito Y, Nakamura T. Hepatocyte growth factor may function as a renotropic factor for regeneration in rats with acute renal injury. American Journal of Physiology. 1993; 265(Renal Fluid Electrolyte Physiol. 34):F61-F69. [PubMed: 8342615]

224. Ilker K, Ilker T, Nigar Y, Recep S, Nermin K, Ahmet O. Iloprost Downregulates Expression of Adhesion Molecules and Reduces Renal Injury Induced by Abdominal Aortic IschemiaReperfusion. Annals of vascular surgery. 2009; 23:212-223. [PubMed: 18774689]

225. Imgrund M, Grone E, Grone H-J, Kretzler M, Holzman L, Schlondorff D, Rothenpieler UW. Reexpression of the developmental gene Pax-2 during experimental acute tubular necrosis in mice1. Kidney Int. 1999; 56:1423-1431. [PubMed: 10504494]

226. Inscho EW, Imig JD, Cook AK, Pollock DM. ETA and ETB receptors differentially modulate afferent and efferent arteriolar responses to endothelin. British Journal of Pharmacology. 2005; 146:1019-1026. [PubMed: 16231007]

227. Ishani A, Xue JL, Himmelfarb J, Eggers PW, Kimmel PL, Molitoris BA, Collins AJ. Acute kidney injury increases risk of ESRD among elderly. J Am Soc Nephrol. 2009; 20:223-228. [PubMed: 19020007]

228. Ishizuka S, Yano T, Hagiwara K, Sone M, Nihei H, Ozasa H, Horikawa S. Extracellular SignalRegulated Kinase Mediates Renal Regeneration in Rats with Myoglobinuric Acute Renal Injury. Biochemical and Biophysical Research Communications. 1999; 254:88-92. [PubMed: 9920737]

229. Iwano M, Plieth D, Danoff TM, Xue C, Okada H, Neilson EG. Evidence that fibroblasts derive from epithelium during tissue fibrosis.[see comment]. Journal of Clinical Investigation. 2002; 110:341-350. [PubMed: 12163453]

230. Iwasaki M, Adachi Y, Minamino K, Suzuki Y, Zhang Y, Okigaki M, Nakano K, Koike Y, Wang J, Mukaide H, Taketani S, Mori Y, Takahashi H, Iwasaka T, Ikehara S. Mobilization of Bone Marrow Cells by G-CSF Rescues Mice from Cisplatin-Induced Renal Failure, and M-CSF Enhances the Effects of G-CSF. Journal of the American Society of Nephrology. 2005; 16:658666. [PubMed: 15689404]

231. Jang H-S, Kim J, Park Y-K, Park KM. Infiltrated Macrophages Contribute to Recovery After Ischemic Injury But Not to Ischemic Preconditioning in Kidneys. Transplantation. 2008; 85:447455. 410.1097/TP.1090b1013e318160f318160d318161. [PubMed: 18301336]

232. Janin A, Deschaumes C, Daneshpouy M, Estaquier J, Micic-Polianski J, Rajagopalan-Levasseur P, Akarid K, Mounier N, Gluckman E, Socie G, Ameisen JC. CD95 engagement induces 
disseminated endothelial cell apoptosis in vivo: immunopathologic implications. Blood. 2002; 99:2940-2947. [PubMed: 11929785]

233. Jefayri MK, Grace PA, Mathie RT. Attenuation of reperfusion injury by renal ischaemic preconditioning: the role of nitric oxide. BJU International. 2000; 85:1007-1013. [PubMed: 10848685]

234. Jensen AM, Norregaard R, Topcu SO, Frokiaer J, Pedersen M. Oxygen tension correlates with regional blood flow in obstructed rat kidney. Journal of Experimental Biology. 2009; 212:31563163. [PubMed: 19749109]

235. Jiang M, Pabla N, Murphy RF, Yang T, Yin X-M, Degenhardt K, White E, Dong Z. Nutlin-3 Protects Kidney Cells during Cisplatin Therapy by Suppressing Bax/Bak Activation. Journal of Biological Chemistry. 2007; 282:2636-2645. [PubMed: 17130128]

236. Jo S-K, Cho WY, Sung SA, Kim HK, Won NH. MEK inhibitor, U0126, attenuates cisplatininduced renal injury by decreasing inflammation and apoptosis. Kidney Int. 2005; 67:458-466. [PubMed: 15673293]

237. Jo SK, Rosner MH, Okusa MD. Pharmacologic Treatment of Acute Kidney Injury: Why Drugs Haven't Worked and What Is on the Horizon. Clinical Journal of the American Society of Nephrology. 2007; 2:356-365. [PubMed: 17699435]

238. Johannes T, Ince C, Klingel K, Unertl KE, Mik EG. Iloprost preserves renal oxygenation and restores kidney function in endotoxemia-related acute renal failure in the rat. Critical Care Medicine. 2009; 37:1423-1432. 1410.1097/CCM.1420b1013e31819b31815f31814e. [PubMed: 19318827]

239. Johnson RJ, Schreiner GF. Hypothesis: the role of acquired tubulointerstitial disease in the pathogenesis of salt-dependent hypertension. Kidney Int. 1997; 52:1169-1179. [PubMed: 9350640]

240. Joyce DE, Gelbert L, Ciaccia A, DeHoff B, Grinnell BW. Gene Expression Profile of Antithrombotic Protein C Defines New Mechanisms Modulating Inflammation and Apoptosis. Journal of Biological Chemistry. 2001; 276:11199-11203. [PubMed: 11278252]

241. Jung KY, Uchida S, Endou H. Nephrotoxicity assessment by measuring cellular ATP content : I. Substrate specificities in the maintenance of ATP content in isolated rat nephron segments. Toxicology and Applied Pharmacology. 1989; 100:369-382. [PubMed: 2551074]

242. Jung YJ, Kim DH, Lee AS, Lee S, Kang KP, Lee SY, Jang KY, Sung MJ, Park SK, Kim W. Peritubular capillary preservation with COMP-angiopoietin-1 decreases ischemia-reperfusioninduced acute kidney injury. American Journal of Physiology - Renal Physiology. 2009; 297:F952-F960. [PubMed: 19656917]

243. Kaizu T, Tamaki T, Tanaka M, Uchida Y, Tsuchihashi S-I, Kawamura A, Kakita A. Preconditioning with tin-protoporphyrin IX attenuates ischemia/reperfusion injury in the rat kidney. Kidney Int. 2003; 63:1393-1403. [PubMed: 12631355]

244. Kakoki M, Hirata Y, Hayakawa H, Suzuki E, Nagata D, Tojo A, Nishimatsu H, Nakanishi N, Hattori Y, Kikuchi K, Nagano T, Omata M. Effects of tetrahydrobiopterin on endothelial dysfunction in rats with ischemic acute renal failure. J Am Soc Nephrol. 2000; 11:301-309. [PubMed: 10665937]

245. Kalluri R, Neilson EG. Epithelial-mesenchymal transition and its implications for fibrosis. Journal of Clinical Investigation. 2003; 112:1776-1784. [PubMed: 14679171]

246. Kanellis J, Paizis K, Cox AJ, Stacker SA, Gilbert RE, Cooper ME, Power DA. Renal ischemiareperfusion increases endothelial VEGFR-2 without increasing VEGF or VEGFR-1 expression. Kidney International. 2002; 61:1696-1706. [PubMed: 11967019]

247. Kang DH, Anderson S, Kim YG, Mazzalli M, Suga S, Jefferson JA, Gordon KL, Oyama TT, Hughes J, Hugo C, Kerjaschki D, Schreiner GF, Johnson RJ. Impaired angiogenesis in the aging kidney: vascular endothelial growth factor and thrombospondin-1 in renal disease. American Journal of Kidney Diseases. 2001; 37:601-611. [PubMed: 11228186]

248. Kang DH, Hughes J, Mazzali M, Schreiner GF, Johnson RJ. Impaired angiogenesis in the remnant kidney model: II. Vascular endothelial growth factor administration reduces renal fibrosis and stabilizes renal function. Journal of the American Society of Nephrology. 2001; 12:1448-1457. [PubMed: 11423573] 
249. Kang DH, Kanellis J, Hugo C, Truong L, Anderson S, Kerjaschki D, Schreiner GF, Johnson R. Role of the microvascular endothelium in progressive renal disease. J Am Soc Nephrol. 2002; 13:806-816. [PubMed: 11856789]

250. Karlberg L, Kallskog O, Norlen BJ, Wolgast M. Postischemic renal failure. Intrarenal blood flow and functional characteristics in the recovery phase. Acta Physiologica Scandinavica. 1982; 115:1-10. [PubMed: 7136794]

251. Karlberg L, Norlen BJ, Ojteg G, Wolgast M. Impaired medullary circulation in postischemic acute renal failure. Acta Physiologica Scandinavica. 1983; 118:11-17. [PubMed: 6624494]

252. Kaushal GP, Kaushal V, Hong X, Shah SV. Role and regulation of activation of caspases in cisplatin-induced injury to renal tubular epithelial cells. Kidney Int. 2001; 60:1726-1736. [PubMed: 11703590]

253. Kellerman PS, Bogusky RT. Microfilament disruption occurs very early in ischemic proximal tubule cell injury. Kidney Int. 1992; 42:896-902. [PubMed: 1453583]

254. Kellum JA. Systematic review: The use of diuretics and dopamine in acute renal failure: a systematic review of the evidence. Critical Care. 1997; 1:53-59. [PubMed: 11094464]

255. Kellum JA, M Decker J. Use of dopamine in acute renal faiulre: a meta analysis. Crit Care Med. 2001; 29:1526-1531. [PubMed: 11505120]

256. Kelly KJ. Distant effects of experimental renal ischemia/reperfusion injury. Journal of the American Society of Nephrology. 2003; 14:1549-1558. [PubMed: 12761255]

257. Kelly KJ, Baird NR, Greene AL. Induction of stress response proteins and experimental renal ischemia/reperfusion. Kidney Int. 2001; 59:1798-1802. [PubMed: 11318950]

258. Kelly KJ, Plotkin Z, Dagher PC. Guanosine supplementation reduces apoptosis and protects renal function in the setting of ischemic injury. The Journal of Clinical Investigation. 2001; 108:12911298. [PubMed: 11696573]

259. Kelly KJ, Sutton TA, Weathered N, Ray N, Caldwell EJ, Plotkin Z, Dagher PC. Minocycline inhibits apoptosis and inflammation in a rat model of ischemic renal injury. Am J Physiol. 2004; 287:F760-F766.

260. Kelly KJ, Williams W, Colvin R, Bonventre JV. Antibody to intercellular adhesion molecule 1 protects the kidney against ischemic injury. Proc Natl Acad Sci. 1994; 18:812-816. [PubMed: 7904759]

261. Kelly KJ, Williams WW Jr, Colvin RB, Bonventre JV. Antibody to intercellular adhesion molecule 1 protects the kidney against ischemic injury. Proceedings of the National Academy of Sciences of the United States of America. 1994; 91:812-816. [PubMed: 7904759]

262. Kelly KJ, Williams WW Jr, Colvin RB, Meehan SM, Springer TA, Gutierrez-Ramos JC, Bonventre JV. Intercellular adhesion molecule-1-deficient mice are protected against ischemic renal injury. Journal of Clinical Investigation. 1996; 97:1056-1063. [PubMed: 8613529]

263. Kerr JFR, Wyllie AH, Currie AR. Apoptosis: a basic biological phenomenon with wide-ranging implications in tissue kinetics. Br J Cancer. 1972; 26:239-257. [PubMed: 4561027]

264. Kerr LE, Birse-Archbold JL, Short DM, McGregor AL, Heron I, MacDonald DC, Thompson J, Carlson GJ, Kelly JS, McCulloch J, Sharkey J. Nucleophosmin is a novel Bax chaperone that regulates apoptotic cell death. Oncogene. 2006; 26:2554-2562. [PubMed: 17072349]

265. Kessler RH. Effects of ischemia on the concentration of adenine nucleotides in the kidney of anesthetized dogs. Proc Soc Exp Biol Med. 1970; 134:1091-1095. [PubMed: 5457736]

266. Kielar ML, John R, Bennett M, Richardson JA, Shelton JM, Chen L, Jeyarajah DR, Zhou XJ, Zhou H, Chiquett B, Nagami GT, Lu CY. Maladaptive Role of IL-6 in Ischemic Acute Renal Failure. Journal of the American Society of Nephrology. 2005; 16:3315-3325. [PubMed: 16192425]

267. Kim J-S, He L, Lemasters JJ. Mitochondrial permeability transition: a common pathway to necrosis and apoptosis. Biochemical and Biophysical Research Communications. 2003; 304:463470. [PubMed: 12729580]

268. Kim J-S, Jin Y, Lemasters JJ. Reactive oxygen species, but not Ca2+ overloading, trigger pHand mitochondrial permeability transition-dependent death of adult rat myocytes after ischemiareperfusion. Am J Physiol Heart Circ Physiol. 2006; 290:H2024-H2034. [PubMed: 16399872] 
269. Kim K, Lee K, Han D, Yu E, Cho Y. Adult stem cell like tubular cells reside in the corticomedullary junction of the kidney. Int J Clin Exp Pathol. 2007; 1:232-241. [PubMed: 18784815]

270. Kim M, Park SW, Kim M, Chen SWC, Gerthoffer WT, D'Agati VD, Lee HT. Selective renal overexpression of human heat shock protein 27 reduces renal ischemiareperfusion injury in mice. American Journal of Physiology - Renal Physiology. 2010; 299:F347-F358. [PubMed: 20484296]

271. Kim YK, Kim HJ, Kwon CH, Kim JH, Woo JS, Jung JS, Kim JM. Role of ERK activation in cisplatin-induced apoptosis in OK renal epithelial cells. Journal of Applied Toxicology. 2005; 25:374-382. [PubMed: 16013042]

272. Kinsey GR, Li L, Okusa MD. Inflammation in Acute Kidney Injury. Nephron Experimental Nephrology. 2008; 109:e102-e107. [PubMed: 18802372]

273. Kinsey GR, McHowat J, Patrick KS, Schnellmann RG. Role of Ca2+-Independent Phospholipase $\mathrm{A} 2 \gamma$ in Ca2+-Induced Mitochondrial Permeability Transition. Journal of Pharmacology and Experimental Therapeutics. 2007; 321:707-715. [PubMed: 17312185]

274. Kinsey GR, Sharma R, Huang L, Li L, Vergis AL, Ye H, Ju S-T, Okusa MD. Regulatory T Cells Suppress Innate Immunity in Kidney Ischemia-Reperfusion Injury. Journal of the American Society of Nephrology. 2009; 20:1744-1753. [PubMed: 19497969]

275. Kjellstrand CJE, Davin T. Time of death, recovery of renal function, development of chronic renal failure and need for chronic hemodialysis in patients with acute tubular necrosis. Trans Am Soc Artif Intern Organs. 1981; 27:45-51. [PubMed: 7331111]

276. Klingebiel T, von Gise H, Bohle A. Morphometric studies on acute renal failure in humans during the oligoanuric and polyuric phases. Clin Nephrol. 1983; 20:1-10. [PubMed: 6883815]

277. Knoll T, Schult S, Birck R, Braun C, Michel MS, Bross S, Juenemann KP, Kirchengast M, Rohmeiss P. Therapeutic administration of an endothelin-A receptor antagonist after acute ischemic renal failure dose-dependently improves recovery of renal function. Journal of Cardiovascular Pharmacology. 2001; 37:483-488. [PubMed: 11300661]

278. Konya L, Szenasi G, Bencsath P, Feher J. Study of the effect of superoxide dismutase on acute renal failure in dogs. Acta Medica Hungarica. 1991; 48:79-85. [PubMed: 1813861]

279. Kramer AA, Postler G, Salhab KF, Mendez C, Carey LC, Rabb H. Renal ischemia/reperfusion leads to macrophage-mediated increase in pulmonary vascular permeability. Kidney Int. 1999; 55:2362-2367. [PubMed: 10354283]

280. Kruger B, Krick S, Dhillon N, Lerner SM, Ames S, Bromberg JS, Lin M, Walsh L, Vella J, Fischereder M, Krämer BK, Colvin RB, Heeger PS, Murphy BT, Schröppel B. Donor Toll-like receptor 4 contributes to ischemia and reperfusion injury following human kidney transplantation. Proceedings of the National Academy of Sciences. 2009; 106:3390-3395.

281. Kruidering M, Van De Water B, De Heer E, Mulder GJ, Nagelkerke JF. Cisplatin-Induced Nephrotoxicity in Porcine Proximal Tubular Cells: Mitochondrial Dysfunction by Inhibition of Complexes I to IV of the Respiratory Chain. Journal of Pharmacology and Experimental Therapeutics. 1997; 280:638-649. [PubMed: 9023274]

282. Kuida K, Haydar T, Kuan C, Gu Y, Taya C, Karasuyama H, Su M, Rakic P, Flavell R. Reduced apoptosis and cytochrome c-mediated caspase activation in mice lacking caspase 9. Cell. 1998; 94:325-337. [PubMed: 9708735]

283. Kwon O, Hong S-M, Sutton TA, Temm CJ. Preservation of peritubular capillary endothelial integrity and increasing pericytes may be critical to recovery from postischemic acute kidney injury. American Journal of Physiology - Renal Physiology. 2008; 295:F351-F359. [PubMed: 18562634]

284. Kwon O, Nelson WJ, Sibley R, Huie P, Scandling JD, Dafoe D, Alfrey E, Myers BD. Backleak, tight junctions, and cell-cell adhesion in postischemic injury to the renal allograft. Journal of Clinical Investigation. 1998; 101:2054-2064. [PubMed: 9593761]

285. Kwon O, Phillips CL, Molitoris BA. Ischemia induces alterations in actin filaments in renal vascular smooth muscle cells. American Journal of Physiology - Renal Fluid \& Electrolyte Physiology. 2002; 282:F1012-F1019. 
286. Lau AH. Apoptosis induced by cisplatin nephrotoxic injury. Kidney Int. 1999; 56:1295-1298. [PubMed: 10504479]

287. Laubach VE, French BA, Okusa MD. Targeting of adenosine receptors in ischemia reperfusion injury. Expert Opinion on Therapeutic Targets. 2011; 15:103-118. [PubMed: 21110787]

288. Lauschke A, Teichgraber UKM, Frei U, Eckardt KU. / Low-dose/' dopamine worsens renal perfusion in patients with acute renal failure. Kidney Int. 2006; 69:1669-1674. [PubMed: 16572117]

289. Leach M, Frank S, Olbrich A, Pfeilschifter J, Thiemermann C. Decline in the expression of copper/zinc superoxide dismutase in the kidney of rats with endotoxic shock: Effects of the superoxide anion radical scavenger, tempol, on organ injury. British Journal of Pharmacology. 1998; 125:817-825. [PubMed: 9831920]

290. Lee DBN, Huang E, Ward HJ. Tight junction biology and kidney dysfunction. American Journal of Physiology - Renal Physiology. 2006; 290:F20-F34. [PubMed: 16339962]

291. Lee HT, Emala CW. Protective effects of renal ischemic preconditioning and adenosine pretreatment: role of A(1) and A(3) receptors. American Journal of Physiology. 2000; 278:F380F387. [PubMed: 10710542]

292. Lee HT, Park SW, Kim M, D'Agati VD. Acute kidney injury after hepatic ischemia and reperfusion injury in mice. Lab Invest. 2008; 89:196-208. [PubMed: 19079326]

293. Lee RH, Pulin AA, Seo MJ, Kota DJ, Ylostalo J, Larson BL, Semprun-Prieto L, Delafontaine P, Prockop DJ. Intravenous hMSCs Improve Myocardial Infarction in Mice because Cells Embolized in Lung Are Activated to Secrete the Anti-inflammatory Protein TSG-6. Cell stem cell. 2009; 5:54-63. [PubMed: 19570514]

294. Lee W-K, Bork U, Gholamrezaei F, Thevenod F. Cd2+-induced cytochrome c release in apoptotic proximal tubule cells: role of mitochondrial permeability transition pore and $\mathrm{Ca} 2+$ uniporter. Am J Physiol Renal Physiol. 2005; 288:F27-F39. [PubMed: 15339793]

295. Leemans JC, Stokman G, Claessen N, Rouschop KM, Teske GJD, Kirschning CJ, Akira S, van der Poll T, Weening JJ, Florquin S. Renal-associated TLR2 mediates ischemia/reperfusion injury in the kidney. The Journal of Clinical Investigation. 2005; 115:2894-2903. [PubMed: 16167081]

296. Lei K, Davis RJ. JNK phosphorylation of Bim-related members of the Bcl2 family induces Baxdependent apoptosis. Proceedings of the National Academy of Sciences of the United States of America. 2003; 100:2432-2437. [PubMed: 12591950]

297. Lemasters J, Qian T, He L, Kim J, Elmore S, Cascio W, Brenner D. Role of mitochondrial inner membrane permeabilization in necrotic cell death, apoptosis, and autophagy. Antioxid Redox Signal. 2002; 4:769-781. [PubMed: 12470504]

298. Lemasters JJ. Mechanisms of Hepatic toxicity V: Necroapoptosis and the mitochondrial permeability transion: shared pathways to necrosis and apoptosis. American Journal of Physiology -Gastrointest Liver Physiol. 1999; 276:G1-G6.

299. Lemasters JJ, Nieminen A-L, Qian T, Trost LC, Elmore SP, Nishimura Y, Crowe RA, Cascio WE, Bradham CA, Brenner DA, Herman B. The mitochondrial permeability transition in cell death: a common mechanism in necrosis, apoptosis and autophagy. Biochimica et Biophysica Acta (BBA) - Bioenergetics. 1998; 1366:177-196.

300. Lemasters JJ, Theruvath TP, Zhong Z, Nieminen A-L. Mitochondrial calcium and the permeability transition in cell death. Biochimica et Biophysica Acta (BBA) - Bioenergetics. 2009; 1787:1395-1401.

301. Lemos FBC, Ijzermans JNM, Zondervan PE, Peeters AMA, van den Engel S, Mol WM, Weimar W, Baan CC. Differential Expression of Heme Oxygenase-1 and Vascular Endothelial Growth Factor in Cadaveric and Living Donor Kidneys after Ischemia-Reperfusion. Journal of the American Society of Nephrology. 2003; 14:3278-3287. [PubMed: 14638927]

302. Leonard EC, Friderich J, Basile DP. VEGF-121 preserves renal microvessel structure and ameliorates secondary renal disease following acute kidney injury. Am J Physiol-Renal Physiol. 2008; 295:F1648-F1657. [PubMed: 18799550]

303. Levy EM, Viscoli CM, Horwitz RI. The effect of acute renal failure on mortality. A cohort analysis. JAMA. 1996; 275:1489-1494. [PubMed: 8622223] 
304. Lewers D, Mathew TH, Maher JF, Schreiner G. Long-term follow-up of renal function and histology after acute tubular necrosis. Annals of Internal Medicine. 1970; 73:523-529. [PubMed: 5506003]

305. Lewis J, Salem M, Chertow GM, Weisberg L, McGrew F, Marbury T, Allgren R. Atrial natriuretic factor in oliguric acute renal failure. American Journal of Kidney Diseases. 2000; 36:767-774. [PubMed: 11007679]

306. Lewy PR, Quintanilla A, Levin NW, Kessler RH. Renal Energy Metabolism and Sodium Reabsorption. Annual Review of Medicine. 1973; 24:365-384.

307. Li B, Cohen A, Hudson TE, Motlagh D, Amrani DL, Duffield JS. Mobilized Human Hematopoietic Stem/Progenitor Cells Promote Kidney Repair After Ischemia/Reperfusion Injury. Circulation. 2010; 121:2211-2220. [PubMed: 20458011]

308. Li F, Mao HP, Ruchalski KL, Wang YH, Choy W, Schwartz JH, Borkan SC. Heat stress prevents mitochondrial injury in ATP-depleted renal epithelial cells. Am J Physiol Cell Physiol. 2002; 283:C917-C926. [PubMed: 12176748]

309. Li H-F, Cheng C-F, Liao W-J, Lin H, Yang R-B. ATF3-Mediated Epigenetic Regulation Protects against Acute Kidney Injury. Journal of the American Society of Nephrology. 2010; 21:10031013. [PubMed: 20360311]

310. Li K, Li Y, Shelton J, Richarsdson J, Spencer E, Chen Z, Wang X, Williams R. Cytochrome c deficiency causes embryonic lethality and attenuates stress induced apoptosis. Cell. 2000; 101:389-399. [PubMed: 10830166]

311. Li L, Huang L, Sung S-sJ, Lobo PI, Brown MG, Gregg RK, Engelhard VH, Okusa MD. NKT Cell Activation Mediates Neutrophil IFN- $\gamma$ Production and Renal Ischemia-Reperfusion Injury. The Journal of Immunology. 2007; 178:5899-5911. [PubMed: 17442974]

312. Li L, Huang L, Sung S-SJ, Vergis AL, Rosin DL, Rose CE Jr, Lobo PI, Okusa MD. The chemokine receptors CCR2 and CX3CR1 mediate monocyte//macrophage trafficking in kidney ischemia-reperfusion injury. Kidney Int. 2008; 74:1526-1537. [PubMed: 18843253]

313. Li LY, Luo X, Wang X. Endonuclease G is an apoptotic DNase when released from mitochondria. Nature. 2001; 412:95-99. [PubMed: 11452314]

314. Liang H, Arsenault J, Mortensen J, Park F, Johnson C, Nilakantan V. Partial attenuation of cytotoxicity and apoptosis by SOD1 in ischemic renal epithelial cells. Apoptosis. 2009; 14:11761189. [PubMed: 19685188]

315. Liangos O, Tighiouart H, Perianayagam MC, Kolyada A, Han WK, Wald R, Bonventre JV, Jaber BL. Comparative analysis of urinary biomarkers for early detection of acute kidney injury following cardiopulmonary bypass. Biomarkers. 2009; 14:423-431. [PubMed: 19572801]

316. Liangos O, Wald R, O'Bell JW, Price L, Pereira BJ, Jaber BL. Epidemiology and Outcomes of Acute Renal Failure in Hospitalized Patients: A National Survey. Clinical Journal of the American Society of Nephrology. 2006; 1:43-51. [PubMed: 17699189]

317. Liano F, Junco E, Pascual J, Madero R, Verde E. The spectrum of acute renal failure in the intensive care unit compared with that seen in other settings. The Madrid Acute Renal Failure Study Group. Kidney Int Suppl. 1998; 66:S16-S24. [PubMed: 9580541]

318. Liano F, Pascual J. Epidemiology of acute renal failure: a prospective, multicenter, communitybased study. Madrid Acute Renal Failure Study Group. Kidney Int. 1996; 50:811-818. [PubMed: 8872955]

319. Lieberthal W, Koh JS, Levine JS. Necrosis and apoptosis in acute renal failure. Seminars in Nephrology. 1998; 18:505-518. [PubMed: 9754603]

320. Lieberthal W, Levine JS. Mechanisms of apoptosis and its potential role in renal tubular epithelial cell injury. American Journal of Physiology. 1996; 271:F477-F488. [PubMed: 8853409]

321. Lieberthal W, Menza SA, Levine JS. Graded ATP depletion can cause necrosis or apoptosis of cultured mouse proximal tubular cells. American Journal of Physiology. 1998; 274:F315-F327. [PubMed: 9486226]

322. Lieberthal W, Nigam SK. Acute renal failure. I. Relative importance of proximal vs. distal tubular injury. Am J Physiol. 1998; 275:F623-F631. [PubMed: 9815122] 
323. Lieberthal W, Nigam SK. Acute renal failure. II. Experimental models of acute renal failure: imperfect but indispensable. American Journal of Physiology - Renal Fluid \& Electrolyte Physiology. 2000; 278:F1-F12.

324. Lin F, Cordes K, Li L, Hood L, Couser WG, Shankland SJ, Igarashi P. Hematopoietic Stem Cells Contribute to the Regeneration of Renal Tubules after Renal Ischemia-Reperfusion Injury in Mice. Journal of the American Society of Nephrology. 2003; 14:1188-1199. [PubMed: 12707389]

325. Lin F, Moran A, Igarashi P. Intrarenal cells, not bone marrow-derived cells are the major source of regeneration of the post-ischemic kidney. Journal of Clinical Investigation. 2005; 115:17561764. [PubMed: 16007252]

326. Lin J-J, Churchill PC, Bidani AK. Theophylline in rats during maintenance phase of postischemic acute renal failure. Kidney Int. 1988; 33:24-28. [PubMed: 3352158]

327. Lin S-L, Li B, Rao S, Yeo E-J, Hudson TE, Nowlin BT, Pei H, Chen L, Zheng JJ, Carroll TJ, Pollard JW, McMahon AP, Lang RA, Duffield JS. Macrophage Wnt7b is critical for kidney repair and regeneration. Proceedings of the National Academy of Sciences. 2010; 107:41944199.

328. Lin S, Chang F, Schrimpf C, Chen Y, Wu C, Wu V, Chiang W, Kuhnert F, Kuo C, Chen Y, Wu $\mathrm{K}$, Tsai T, duffield J. Targetting endothelium pericyte cross talk by inhibiting VEGF receptor signaling attenuates kidney microvascular rarefaction and fibrosis. Am J Pathol. 2011; 178:911923. [PubMed: 21281822]

329. Linas S, Whittenburg D, Repine JE. Nitric oxide prevents neutrophil-mediated acute renal failure. American Journal of Physiology - Renal Physiology. 1997; 272:F48-F54.

330. Linas SL, Whittenburg D, Parsons PE, Repine JE. Ischemia increases neutrophil retention and worsens acute renal failure: Role of oxygen metabolites and ICAM-1. Kidney Int. 1995; 48:1584-1591. [PubMed: 8544418]

331. Ling W, Zhaohui N, Ben H, Leyi G, Jianping L, Huili D, Jiaqi Q. Urinary IL-18 and NGAL as Early Predictive Biomarkers in Contrast-Induced Nephropathy after Coronary Angiography. Nephron Clinical Practice. 2008; 108:c176-c181. [PubMed: 18287807]

332. Liu J-P, Baker J, Perkins AS, Robertson EJ, Efstratiadis A. Mice carrying null mutations of the genes encoding insulin-like growth factor I (Igf-1) and type 1 IGF receptor (Igf1r). Cell. 1993; 75:59-72. [PubMed: 8402901]

333. Liu L, Yang C, Herzog C, Seth R, Kaushal G. Proteosome inhibitors prevent cisplatin induced mitochondrial release of apoptosis inducing factor and markedly ameliorate cisplatin nephrotoxicity. Biochemical pharmacology. 2010; 79:137-146. [PubMed: 19699182]

334. Liu M, Grigoryev DN, Crow MT, Haas M, Yamamoto M, Reddy SP, Rabb H. Transcription factor $\mathrm{Nrf} 2$ is protective during ischemic and nephrotoxic acute kidney injury in mice. Kidney Int. 2009; 76:277-285. [PubMed: 19436334]

335. Liu M, Liang Y, Chigurupati S, Lathia JD, Pletnikov M, Sun Z, Crow M, Ross CA, Mattson MP, Rabb H. Acute Kidney Injury Leads to Inflammation and Functional Changes in the Brain. Journal of the American Society of Nephrology. 2008; 19:1360-1370. [PubMed: 18385426]

336. Lo LJ, Go AS, Chertow GM, McCulloch CE, Fan D, Ordonez JD, Hsu CY. Dialysis-requiring acute renal failure increases the risk of progressive chronic kidney disease. Kidney Int. 2009; 76:893-899. [PubMed: 19641480]

337. Lopez-Novoa JM. Potential role of platelet activating factor in acute renal failure. Kidney Int. 1999; 55:1672-1682. [PubMed: 10231429]

338. Lopez-Novoa JM, Quiros Y, Vicente L, Morales AI, Lopez-Hernandez FJ. New insights into the mechanism of aminoglycoside nephrotoxicity: an integrative point of view. Kidney Int. 79:3345. [PubMed: 20861826]

339. Loukogeorgakis SP, Williams R, Panagiotidou AT, Kolvekar SK, Donald A, Cole TJ, Yellon DM, Deanfield JE, MacAllister RJ. Transient Limb Ischemia Induces Remote Preconditioning and Remote Postconditioning in Humans by a KATP Channel Dependent Mechanism. Circulation. 2007; 116:1386-1395. [PubMed: 17724264]

340. Lovis C, Mach F, Donati YR, Bonventre JV, Polla BS. Heat shock proteins and the kidney. Renal Failure. 1994; 16:179-192. [PubMed: 8041958] 
341. Ma D, Lim T, Xu J, Tang H, Wan Y, Zhao H, Hossain M, Maxwell PH, Maze M. Xenon Preconditioning Protects against Renal Ischemic-Reperfusion Injury via HIF-1a Activation. Journal of the American Society of Nephrology. 2009; 20:713-720. [PubMed: 19144758]

342. Ma H, Saenko M, Opuko A, Togawa A, Soda K, Marlier A, Moeckel GW, Cantley LG, Ishibe S. Deletion of the Met receptor in the collecting duct decreases renal repair following ureteral obstruction. Kidney Int. 2009; 76:868-876. [PubMed: 19675527]

343. MacNider W. The functional and pathological response of the kidney in dogs subjected to a second subcutaneous injection of uranium nitrate. Journal of Experimental Medicine. 1929; 49:411-433. [PubMed: 19869554]

344. Madesh M, Hajnoczky G. VDAC-dependent permeabilization of the outer mitochondrial membrane by superoxide induces rapid and massive cytochrome c release. The Journal of Cell Biology. 2001; 155:1003-1016. [PubMed: 11739410]

345. Maeshima A. Label-retaining cells in the kidney: origin of regenerating cells after renal ischemia. Clinical and Experimental Nephrology. 2007; 11:269-274. [PubMed: 18085386]

346. Maeshima A, Yamashita S, Nojima Y. Identification of Renal Progenitor-Like Tubular Cells that Participate in the Regeneration Processes of the Kidney. Journal of the American Society of Nephrology. 2003; 14:3138-3146. [PubMed: 14638912]

347. Maines MD, Mayer RD, Ewing JF, McCoubrey WK. Induction of kidney heme oxygenase-1 (HSP32) mRNA and protein by ischemia/reperfusion: possible role of heme as both promotor of tissue damage and regulator of HSP32. Journal of Pharmacology and Experimental Therapeutics. 1993; 264:457-462. [PubMed: 8423544]

348. Mandel L, Doctor R, Bacallao R. ATP depletion: a novel method to study junctional properties in epithelial tissues. II. Internalization of $\mathrm{Na}+\mathrm{K}(+)$-ATPase and Ecadherin. J Cell Sci. 1994; 107:3315-3324. [PubMed: 7706388]

349. Manley GT, Binder DK, Papadopoulos MC, Verkman AS. New insights into water transport and edema in the central nervous system from phenotype analysis of aquaporin-4 null mice. Neuroscience. 2004; 129:981-989.

350. Mao H, Wang Y, Li Z, Ruchalski KL, Yu X, Schwartz JH, Borkan SC. Hsp72 Interacts with Paxillin and Facilitates the Reassembly of Focal Adhesions during Recovery from ATP Depletion. Journal of Biological Chemistry. 2004; 279:15472-15480. [PubMed: 14718530]

351. Martinez-Salgado C, Eleno N, Tavares P, Rodriguez-Barbero A, Garcia-Criado J, Bolanos JP, Lopez-Novoa JM. Involvement of reactive oxygen species on gentamicininduced mesangial cell activation. Kidney Int. 2002; 62:1682-1692. [PubMed: 12371968]

352. Marumo T, Hishikawa K, Yoshikawa M, Fujita T. Epigenetic Regulation of BMP7 in the Regenerative Response to Ischemia. Journal of the American Society of Nephrology. 2008; 19:1311-1320. [PubMed: 18322163]

353. Mason J, Beck F, Dorge A, Rick R, Thurau K. Intracellular electrolyte composition following renal ischemia. Kidney Int. 1981; 20:61-70. [PubMed: 7300114]

354. Mason J, Torhorst J, Welsch J. Role of the medullary perfusion defect in the pathogenesis of ischemic renal failure. Kidney Int. 1984; 26:283-293. [PubMed: 6513274]

355. Massicot F, Martin C, Dutertre-Catella H, Ellouk-Achard S, Pham-Huy C, Thevenin M, Rucay P, Warnet J-M, Claude J-R. Modulation of energy status and cytotoxicity induced by FK506 and cyclosporin A in a renal epithelial cell line. Archives of Toxicology. 1997; 71:529-531. [PubMed: 9248632]

356. Matejka GL, Jennische E. IGF-I binding and IGF-I mRNA expression in the post-ischemic regenerating rat kidney. Kidney Int. 1992; 42:1113-1123. [PubMed: 1453597]

357. Matsuyama M, Yoshimura R, Akioka K, Okamoto M, Ushigome H, Kadotani Y, Nakatani T, Yoshimura M. Tissue factor antisense oligonucleotides prevent renal ischema reperfusion injury. Tranplantation. 2003; 76:786-791.

358. Matthys E, Patton MK, Osgood RW, Venkatachalam MA, Stein JH. Alterations in vascular function and morphology in acute ischemic renal failure. Kidney Int. 1983; 23:717-724. [PubMed: 6876567] 
359. Mattson DL, Lu S, Cowley AW Jr. Role of nitric oxide in the control of the renal medullary circulation. Clinical \& Experimental Pharmacology \& Physiology. 1997; 24:587-590. [PubMed: 9269532]

360. Megyesi J, Andrade L, Vieira JM Jr, Safirstein RL, Price PM. Positive effect of the induction of p21WAF1/CIP1 on the course of ischemic acute renal failure. Kidney International. 2001; 60:2164-2172. [PubMed: 11737590]

361. Megyesi J, Di Mari J, Udvarhelyi N, Price PM, Safirstein R. DNA synthesis is dissociated from the immediate-early gene response in the post-ischemic kidney. Kidney Int. 1995; 48:1451-1458. [PubMed: 8544401]

362. Megyesi J, Safirstein RL, Price PM. Induction of p21WAF1/CIP1/SDI1 in kidney tubule cells affects the course of cisplatin-induced acute renal failure. Journal of Clinical Investigation. 1998; 101:777-782. [PubMed: 9466972]

363. Mehta RL, Chertow GM. Acute renal failure definitions and classification: time for change? J Am Soc Nephrol. 2003; 14:2178-2187. [PubMed: 12874474]

364. Melk A, Schmidt BMW, Vongwiwatana A, Rayner DC, Halloran PF. Increased Expression of Senescence-Associated Cell Cycle Inhibitor p16INK4a in Deteriorating Renal Transplants and Diseased Native Kidney. American Journal of Transplantation. 2005; 5:1375-1382. [PubMed: 15888044]

365. Melnikov VY, Ecder T, Fantuzzi G, Siegmund B, Lucia MS, Dinarello CA, Schrier RW, Edelstein CL. Impaired IL-18 processing protects caspase-1 deficient mice from ischemic acute renal failure. The Journal of Clinical Investigation. 2001; 107:1145-1152. [PubMed: 11342578]

366. Melnikov VY, Faubel S, Siegmund B, Lucia MS, Ljubanovic D, Edelstein CL. Neutrophilindependent mechanisms of caspase-1 and IL-18 mediated ischemic acute tubular necrosis in mice. The Journal of Clinical Investigation. 2002; 110:1083-1091. [PubMed: 12393844]

367. Michal JT, Julio PJ, Joseph PG, Anthony JC, Allan WA, Govindarajan R, Keith LK, Andrew DB, Matthew DG, Jawed A, Karl AN. Renal Hemodynamic, Inflammatory, and Apoptotic Responses to Lipopolysaccharide in HO-1-/- Mice. The American journal of pathology. 2007; 170:1820 1830. [PubMed: 17525251]

368. Mikhailov V, Mikhailova M, Pulkrabek DJ, Dong Z, Venkatachalam MA, Saikumar P. Bcl-2 Prevents Bax Oligomerization in the Mitochondrial Outer Membrane. Journal of Biological Chemistry. 2001; 276:18361-18374. [PubMed: 11279112]

369. Miller SB, Martin DR, Kissane J, Hammerman MR. Hepatocyte growth factor accelerates recovery from acute ischemic renal injury in rats. American Journal of Physiology. 1994; 266(Renal Fluid Electrolye Physiol. 35):F129-F134. [PubMed: 8304478]

370. Miller SB, Martin DR, Kissane J, Hammerman MR. Insulin-like growth factor I accelerates recovery from ischemic acute tubular necrosis in the rat. Proceedings of the National Academy of Sciences of the United States of America. 1992; 89:11876-11880. [PubMed: 1465411]

371. Miller SB, Martin DR, Kissane J, Hammerman MR. Rat models for clinical use of insulin-like growth factor I in acute renal failure. American Journal of Physiology. 1994; 266(Renal Fluid Electrolyte Physiol. 35):F949-F956. [PubMed: 8023974]

372. Miller TR, Anderson RJ, Linas SL, Henrich WL, Berns AS, Gabow PA, Schrier RW. Urinary diagnostic indices in acute renal failure: a prospective study. Ann Intern Med. 1978; 89:47-50. [PubMed: 666184]

373. Misao J, Hayakawa Y, Ohno M, Kato S, Fujiwara T, Fujiwara H. Expression of bcl-2 Protein, an Inhibitor of Apoptosis, and Bax, an Accelerator of Apoptosis, in Ventricular Myocytes of Human Hearts With Myocardial Infarction. Circulation. 1996; 94:1506-1512. [PubMed: 8840837]

374. Mitazaki S, Kato N, Suto M, Hiraiwa K, Abe S. Interleukin-6 deficiency accelerates cisplatininduced acute renal failure but not systemic injury. Toxicology. 2009; 265:115-121. [PubMed: 19833167]

375. Miyazawa S, Watanabe H, Miyaji C, Hotta O, Abo T. Leukocyte accumulation and changes in extra-renal organs during renal ischemia reperfusion in mice. Journal of Laboratory and Clinical Medicine. 2002; 139:269-278. [PubMed: 12032487]

376. Molitoris BA. Actin cytoskeleton in ischemic acute renal failure. Kidney Int. 2004; 66:871-883. [PubMed: 15253754] 
377. Molitoris BA. Ischemia-induced loss of epithelial polarity: potential role of the actin cytoskeleton. American Journal of Physiology - Renal Physiology. 1991; 260:F769-F778.

378. Molitoris BA. Putting the actin cytoskeleton into perspective: pathophysiology of ischemic alterations. American Journal of Physiology. 1997; 272:F430-F433. [PubMed: 9140042]

379. Molitoris BA, Chan LK, Shapiro JI, Conger JD, Falk SA. Loss of epithelial polarity: a novel hypothesis for reduced proximal tubule $\mathrm{Na}+$ transport following ischemic injury. Journal of Membrane Biology. 1989; 107:119-127. [PubMed: 2541248]

380. Molitoris BA, Falk SA, Dahl RH. Ischemia-induced loss of epithelial polarity. Role of the tight junction. Journal of Clinical Investigation. 1989; 84:1334-1339. [PubMed: 2551926]

381. Molitoris, BA.; Finn, WF. Acute renal failure : a companion to Brenner and Rector's The kidney. Philadelphia: Saunders; 2001. p. xiv, 535 p., 538 p. of plates

382. Molitoris BA, Geerdes A, McIntosh JR. Dissociation and redistribution of $\mathrm{Na}+\mathrm{K}(+)$-ATPase from its surface membrane actin cytoskeletal complex during cellular ATP depletion. Journal of Clinical Investigation. 1991; 88:462-469. [PubMed: 1650794]

383. Molitoris BA, Sandoval R, Sutton TA. Endothelial injury and dysfunction in ischemic acute renal failure. Critical Care Medicine. 2002; 30:S235-S240. [PubMed: 12004242]

384. Morgera S, Kraft AK, Siebert G, Luft FC, Neumayer HH. Long-term outcomes in acute renal failure patients treated with continuous renal replacement therapies. Am J Kidney Dis. 2002; 40:275-279. [PubMed: 12148099]

385. Morigi M, Rota C, Montemurro T, Montelatici E, Lo Cicero V, Imberti B, Abbate M, Zoja C, Cassis P, Longaretti L, Rebulla P, Introna M, Capelli C, Benigni A, Remuzzi G, Lazzari L. LifeSparing Effect of Human Cord Blood-Mesenchymal Stem Cells in Experimental Acute Kidney Injury. STEM CELLS. 2010; 28:513-522. [PubMed: 20049901]

386. Mortensen J, Shames B, Johnson C, Nilakantan V. MnTMPyP, a superoxide dismutase/catalase mimetic, decreases inflammatory indices in ischemic acute kidney injury. Inflammation Research. :1-9.

387. Muramatsu Y, Tsujie M, Kohda Y, Pham B, Perantoni AO, Zhao H, Jo SK, Yuen PS, Craig L, Hu $\mathrm{X}$, Star RA. Early detection of cysteine rich protein 61 (CYR61, CCN1) in urine following renal ischemic reperfusion injury. Kidney Int. 2002; 62:1601-1610. [PubMed: 12371960]

388. Nagothu KK, Bhatt R, Kaushal GP, Portilla D. Fibrate prevents cisplatininduced proximal tubule cell death. Kidney Int. 2005; 68:2680-2693. [PubMed: 16316343]

389. Nash K, Hafeez A, Hou S. Hospital-acquired renal insufficiency. Am J Kidney Dis. 2002; 39:930-936. [PubMed: 11979336]

390. Nash K, Hafeez A, Hou S. Hospital-acquired renal insufficiency. American journal of kidney diseases : the official journal of the National Kidney Foundation. 2002; 39:930-936. [PubMed: 11979336]

391. Nath K, Haggard J, Croatt A, Grande J, Poss K, Alam J. The Indispensability of Heme Oxygenase-1 in Protecting against Acute Heme Protein-Induced Toxicity in Vivo. The American journal of pathology. 2000; 156:1527-1535. [PubMed: 10793064]

392. Nath K, Norby S. Reactive oxygen species and acute renal failure. The American journal of medicine. 2000; 109:665-678. [PubMed: 11099687]

393. Nath KA. Heme oxygenase-1: A provenance for cytoprotective pathways in the kidney and other tissues. Kidney Int. 2006; 70:432-443. [PubMed: 16775600]

394. Nath KA, Croatt AJ, Haggard JJ, Grande JP. Renal response to repetitive exposure to heme proteins: Chronic injury induced by an acute insult. Kidney Int. 2000; 57:2423-2433. [PubMed: 10844611]

395. Nemoto T, Burne MJ, Daniels F, O'Donnell MP, Crosson J, Berens K, Issekutz A, Kasiske BL, Keane WF, Rabb H. Small molecule selectin ligand inhibition improves outcome in ischemic acute renal failure. Kidney Int. 2001; 60:2205-2214. [PubMed: 11737594]

396. Neto JS, Nakao A, Kimizuka K, Romanosky AJ, Stolz DB, Uchiyama T, Nalesnik MA, Otterbein LE, Murase N. Protection of transplant-induced renal ischemia-reperfusion injury with carbon monoxide. American Journal of Physiology - Renal Physiology. 2004; 287:F979-F989.

[PubMed: 15292046] 
397. Nicolli A, Basso E, Petronilli V, Wenger RM, Bernardi P. Interactions of Cyclophilin with the Mitochondrial Inner Membrane and Regulation of the Permeability Transition Pore, a Cyclosporin A-sensitive Channel. Journal of Biological Chemistry. 1996; 271:2185-2192. [PubMed: 8567677]

398. Nitescu N, Ricksten S-E, Marcussen N, Haraldsson Br, Nilsson U, Basu S, Guron G. Nacetylcysteine attenuates kidney injury in rats subjected to renal ischaemiareperfusion. Nephrology Dialysis Transplantation. 2006; 21:1240-1247.

399. Nogae S, Miyazaki M, Kobayashi N, Saito T, Abe K, Saito H, Nakane P, Nakanishi Y, Koji T. Induction of apoptosis in ischemia-reperfusion model of mouse kidney: possible involvement of Fas. J Am Soc Nephrol. 1998; 9:620-631. [PubMed: 9555665]

400. Noiri E, Gailit J, Sheth D, Magazine H, Gurrath M, Muller G, Kessler H, Goligorsky MS. Cyclic RGD peptides ameliorate ischemic acute renal failure in rats. Kidney Int. 1994; 46:1050-1058. [PubMed: 7861698]

401. Nolan CV, Shaikh ZA. Lead nephrotoxicity and associated disorders: biochemical mechanisms. Toxicology. 1992; 73:127-146. [PubMed: 1319092]

402. Nony PA, Schnellmann RG. Mechanisms of Renal Cell Repair and Regeneration after Acute Renal Failure. Journal of Pharmacology and Experimental Therapeutics. 2003; 304:905-912. [PubMed: 12604664]

403. Norman J, Badie-Dezfooly B, Nord EP, Kurtz I, Schlosser J, Chaudhari A, Fine LG. EGFinduced mitogenesis in proximal tubular cells: potentiation by angiotensin II. American Journal of Physiology - Renal Physiology. 1987; 253:F299-F309.

404. Norman J, Fine LG. Intrarenal oxygenation in chronic renal failure. Clinical \& Experimental Pharmacology \& Physiology. 2006; 33:989-996. [PubMed: 17002678]

405. O'Shea MH, Miller SB, Hammerman MR. Effects of IGF-I on renal function in patients with chronic renal failure. American Journal of Physiology - Renal Physiology. 1993; 264:F917F922.

406. Ogata M, Iwamoto T, Tazawa N, Nishikawa M, Yamashita J, Takaoka M, Matsumura Y. A novel and selective $\mathrm{Na}+\mathrm{Ca} 2+$ exchange inhibitor, SEA0400, improves ischemia/reperfusion-induced renal injury. European Journal of Pharmacology. 2003; 478:187-198. [PubMed: 14575804]

407. Oh D-J, Dursun B, He Z, Lu L, Hoke TS, Ljubanovic D, Faubel S, Edelstein CL. Fractalkine receptor (CX3CR1) inhibition is protective against ischemic acute renal failure in mice. American Journal of Physiology - Renal Physiology. 2008; 294:F264-F271. [PubMed: 18003857]

408. Okada H, Danoff TM, Kalluri R, Neilson EG. Early role of Fsp1 in epithelialmesenchymal transformation. American Journal of Physiology. 1997; 273:F563-F574. [PubMed: 9362334]

409. Okusa MD, Linden J, Huang L, Rosin DL, Smith DF, Sullivan G. Enhanced protection from renal ischemia: Reperfusion injury with A2A-adenosine receptor activation and PDE 4 inhibition. Kidney Int. 2001; 59:2114-2125. [PubMed: 11380813]

410. Oliver J, Mac DM, Tracy A. The pathogenesis of acute renal failure associated with traumatic and toxic injury; renal ischemia, nephrotoxic damage and the ischemic episode. J Clin Invest. 1951; 30:1307-1439. [PubMed: 14897900]

411. Oliver JA, Maarouf O, Cheema FH, Martens TP, Al-Awqati Q. The renal papilla is a niche for adult kidney stem cells. The Journal of Clinical Investigation. 2004; 114:795-804. [PubMed: 15372103]

412. Oltval ZN, Milliman CL, Korsmeyer SJ. Bcl-2 heterodimerizes in vivo with a conserved homolog, Bax, that accelerates programed cell death. Cell. 1993; 74:609-619. [PubMed: 8358790]

413. Ortiz A, Lorz C, Egido J. The Fas ligand/Fas system in renal injury. Nephrol Dial Transplant. 1999; 14:1831-1834. [PubMed: 10462254]

414. Ouellette AJ, Malt RA, Sukhatme VP, Bonventre JV. Expression of two "immediate early" genes, Egr-1 and c-fos, in response to renal ischemia and during compensatory renal hypertrophy in mice. J Clin Invest. 1990; 85:766-771. [PubMed: 2107209] 
415. Padanilam BJ. Cell death induced by acute renal injury: a perspective on the contributions of apoptosis and necrosis. American Journal of Physiology - Renal Fluid \& Electrolyte Physiology. 2003; 284:F608-F627.

416. Pagtalunan M, Olson J, Tilney N, Meyer T. Late consequences of acute ischemic injury to a solitary kidney. J Am Soc Nephrol. 1999; 10:366-373. [PubMed: 10215337]

417. Paller MS. Free radical scavengers in mercuric chloride-induced acute renal failure in the rat. Journal of Laboratory \& Clinical Medicine. 1985; 105:459-463. [PubMed: 3920337]

418. Paller MS. Renal work, glutathione and susceptibility to free radical-mediated postischemic injury. Kidney Int. 1988; 33:843-849. [PubMed: 3386136]

419. Paller MS, Hedlund BE. Extracellular iron chelators protect kidney cells from hypoxia/ reoxygenation. Free Radical Biology and Medicine. 1994; 17:597-603. [PubMed: 7532608]

420. Paller MS, Hedlund BE. The role of iron in postischemic renal failure in the rat. Kidney Int. 1988; 34:474-480. [PubMed: 3143849]

421. Paller MS, Hoidal JR, Ferris TF. Oxygen free radicals in ischemic acute renal failure in the rat. Journal of Clinical Investigation. 1984; 74:1156-1164. [PubMed: 6434591]

422. Parikh CR, Devarajan P. New biomarkers of acute kidney injury. Critical Care Medicine. 2008; 36:S159-S165. [PubMed: 18382188]

423. Park H-C, Yasuda K, Kuo M-C, Ni J, Ratliff B, Chander P, Goligorsky MS. Renal capsule as a stem cell niche. American Journal of Physiology - Renal Physiology. 2010; 298:F1254-F1262. [PubMed: 20200095]

424. Park K, Chen A, Bonventre JV. Prevention of kidney ischemia/reperfusion induced functional injury and JNK, p38 and MAPK kinase activation by remote ischemic pretreatment. Journal of Biological Chemistry. 2001; 276:11870-11876. [PubMed: 11150293]

425. Park KM, Kramers C, Vayssier-Taussat M, Chen A, Bonventre JV. Prevention of kidney ischemia/reperfusion-induced functional injury, MAPK and MAPK kinase activation, and inflammation by remote transient ureteral obstruction. Journal of Biological Chemistry. 2002; 277:2040-2049. [PubMed: 11696540]

426. Patschan D, Krupincza K, Patschan S, Zhang Z, Hamby C, Goligorsky MS. Dynamics of mobilzation and homing of endothelial progenitor cells after acute renal ischemia: modulation by ischemic preconditioning. Am J Physiol. 2006; 291:F176-F185.

427. Patschan D, Patschan S, Gobe GG, Chintala S, Goligorsky MS. Uric Acid Heralds Ischemic Tissue Injury to Mobilize Endothelial Progenitor Cells. Journal of the American Society of Nephrology. 2007; 18:1516-1524. [PubMed: 17409313]

428. Pechman K, Basile DP, Lund H, Mattson DL. Immune suppression blocks sodium sensitive hypertension following recovery from acute renal failure. Am J Physiol Regulatory Integrative. 2008; 294:R1234-R1239.

429. Pechman KR, De Miguel C, Lund H, Leonard EC, Basile DP, Mattson DL. Recovery from renal ischemia-reperfusion injury is associated with altered renal hemodynamics, blunted pressure natriuresis, and sodium-sensitive hypertension. Am J Physiol Regul Integr Comp Physiol. 2009; 297:R1358-R1363. [PubMed: 19710386]

430. Peralta C, Perales JC, Bartrons R, Mitchell C, Gilgenkrantz H, Xaus C, Prats N, Fernandez L, Gelpi E, Panes J, Rosello-Catafau J. The Combination of Ischemic Preconditioning and Liver Bcl-2 Overexpression Is a Suitable Strategy to Prevent Liver and Lung Damage after Hepatic Ischemia-Reperfusion. Am J Pathol. 2002; 160:2111-2122. [PubMed: 12057915]

431. Perianayagam MC, Liangos O, Kolyada AY, Wald R, MacKinnon RW, Li L, Rao M, Balakrishnan VS, Bonventre JV, Pereira BJ, Jaber BL. NADPH Oxidase p22phox and Catalase Gene Variants Are Associated with Biomarkers of Oxidative Stress and Adverse Outcomes in Acute Renal Failure. Journal of the American Society of Nephrology. 2007; 18:255-263. [PubMed: 17151330]

432. Periyasamy-Thandavan S, Jiang M, Schoenlein P, Dong Z. Autophagy: molecular machinery, regulation, and implications for renal pathophysiology. American Journal of Physiology - Renal Physiology. 2009; 297:F244-F256. [PubMed: 19279132] 
433. Periyasamy-Thandavan S, Jiang M, Wei Q, Smith R, Yin X-M, Dong Z. Autophagy is cytoprotective during cisplatin injury of renal proximal tubular cells. Kidney Int. 2008; 74:631640. [PubMed: 18509315]

434. Peterson O, Gabbai F, Myers R, Mizisin A, Blantz RC. A single nephron model of acute tubular injury: role of tubularglomerular feedback. Kidney Int. 1989; 36:1037-1044. [PubMed: 2601254]

435. Petrinec D, Reilly JM, Sicard GA, Lowell JA, Howard TK, Martin DR, Brennan DC, Miller SB. Insulin-like growth factor-I attenuates delayed graft function in a canine renal autotransplantation model. Surgery. 1996; 120:221-226. [PubMed: 8751586]

436. Piechota M, Banach M, Irzmanski R, Barylski M, Piechota-Urbanska M, Kowalski J, Pawlicki L. Plasma Endothelin-1 Levels in Septic Patients. Journal of Intensive Care Medicine. 2007; 22:232-239. [PubMed: 17722367]

437. Pittock ST, Norby SM, Grande JP, Croatt AJ, Bren GD, Badley AD, Caplice NM, Griffin MD, Nath KA. MCP-1 is up-regulated in unstressed and stressed HO-1 knockout mice: Pathophysiologic correlates1. Kidney Int. 2005; 68:611-622. [PubMed: 16014038]

438. Plotnikov EY, Kazachenko AV, Vyssokikh MY, Vasileva AK, Tcvirkun DV, Isaev NK, Kirpatovsky VI, Zorov DB. The role of mitochondria in oxidative and nitrosative stress during ischemia//reperfusion in the rat kidney. Kidney Int. 2007; 72:1493-1502. [PubMed: 17914353]

439. Pritchard KA, Ackerman AW, Gross ER, Stepp DW, Shi Y, Fontana JT, Baker JE, Sessa WC. Heat Shock Protein 90 Mediates the Balance of Nitric Oxide and Superoxide Anion from Endothelial Nitric-oxide Synthase. Journal of Biological Chemistry. 2001; 276:17621-17624. [PubMed: 11278264]

440. Qian T, Nieminen A-L, Herman B, Lemasters JJ. Mitochondrial permeability transition in pHdependent reperfusion injury to rat hepatocytes. Am J Physiol Cell Physiol. 1997; 273:C1783C1792.

441. Quigg RJ. Complement and the Kidney. The Journal of Immunology. 2003; 171:3319-3324. [PubMed: 14500622]

442. Quiros Y, Vicente-Vicente L, Morales AI, Lopez-Novoa J, Lopez-Hernandez FJ. An Integrative Overview on the Mechanisms Underlying the Renal Tubular Cytotoxicity of Gentamicin. Toxicological Sciences. 2011; 119:245-256. [PubMed: 20829429]

443. Rabb H. Immune Modulation of Acute Kidney Injury. Journal of the American Society of Nephrology. 2006; 17:604-606. [PubMed: 16481410]

444. Rabb H, Mendiola CC, Dietz J, Saba SR, Issekutz TB, Abanilla F, Bonventre JV, Ramirez G. Role of CD11a and CD11b in ischemic acute renal failure in rats. American Journal of Physiology. 1994; 267:F1052-F1058. [PubMed: 7810691]

445. Rabb H, Mendiola CC, Saba SR, Dietz JR, Smith CW, Bonventre JV, Ramirez G. Antibodies to ICAM-1 protect kidneys in severe ischemic reperfusion injury. Biochemical \& Biophysical Research Communications. 1995; 211:67-73. [PubMed: 7779111]

446. Rabb H, O'Meara YM, Maderna P, Coleman P, Brady HR. Leukocytes, cell adhesion molecules and ischemic acute renal failure. Kidney Int. 1997; 51:1463-1468. [PubMed: 9150459]

447. Racusen LC. The histopathology of acute renal failure. New Horizons. 1995; 3:662-668. [PubMed: 8574596]

448. Rajdev S, Hara K, Kokubo Y, Mestril R, Dillmann W, Weinstein PR, Sharp FR. Mice overexpressing rat heat shock protein 70 are protected against cerebral infarction. Annals of Neurology. 2000; 47:782-791. [PubMed: 10852544]

449. Rajesh KK, Torsten Toftgard N, Andrew NR. Translation of remote ischaemic preconditioning into clinical practice. The Lancet. 2009; 374:1557-1565.

450. Ramesh G, Reeves WB. TNFR2-mediated apoptosis and necrosis in cisplatininduced acute renal failure. American Journal of Physiology - Renal Physiology. 2003; 285:F610-F618. [PubMed: 12865254]

451. Ramirez V, Mejia-Vilet JM, Hernandez D, Gamba G, Bobadilla NA. Radicicol, a heat shock protein 90 inhibitor, reduces glomerular filtration rate. American Journal of Physiology - Renal Physiology. 2008; 295:F1044-F1051. [PubMed: 18667483]

452. Rasbach KA, Schnellmann RG. Isoflavones Promote Mitochondrial Biogenesis. Journal of Pharmacology and Experimental Therapeutics. 2008; 325:536-543. [PubMed: 18267976] 
453. Rasbach KA, Schnellmann RG. PGC-1[alpha] over-expression promotes recovery from mitochondrial dysfunction and cell injury. Biochemical and Biophysical Research Communications. 2007; 355:734-739. [PubMed: 17307137]

454. Ratcliffe PJ, Moonen CTW, Holloway PAH, Ledingham JGG, Radda GK. Acute renal failure in hemorrhagic hypotension: Cellular energetics and renal function. Kidney Int. 1986; 30:355-360. [PubMed: 3784280]

455. Reinders MEJ, Rabelink TJ, Briscoe DM. Angiogenesis and Endothelial Cell Repair in Renal Disease and Allograft Rejection. Journal of the American Society of Nephrology. 2006; 17:932942. [PubMed: 16481411]

456. Rhoden E, Telöken C, Lucas M, Rhoden C, Mauri M, Zettler C, Belló-Klein A, Barros E. Protective effect of allopurinol in the renal ischemia-reperfusion in uninephrectomized rats. General Pharmacology: The Vascular System. 2000; 35:189-193.

457. Rifkin IR, Leadbetter EA, Busconi L, Viglianti G, Marshak-Rothstein A. Toll-like receptors, endogenous ligands, and systemic autoimmune disease. Immunological Reviews. 2005; 204:2742. [PubMed: 15790348]

458. Robinson JWL, Mirkovitch V, Gomba Sz. Alterations in the dog renal tubular epithelium during normothermic ischemia. Kidney Int. 1977; 11:86-92. [PubMed: 846066]

459. Romanov V, Noiri E, Czerwinski G, Finsinger D, Kessler H, Goligorsky MS. Two novel probes reveal tubular and vascular Arg-Gly-Asp (RGD) binding sites in the ischemic rat kidney. Kidney Int. 1997; 52:93-102. [PubMed: 9211351]

460. Rookmaaker MB, Verhaar MC, van Zonneveld AJ, Rabelink TJ. Progenitor cells in the kidney: biology and therapeutic perspectives. Kidney Int. 2004; 66:518-522. [PubMed: 15253701]

461. Ruchalski K, Mao H, Singh SK, Wang Y, Mosser DD, Li F, Schwartz JH, Borkan SC. HSP72 inhibits apoptosis-inducing factor release in ATP-depleted renal epithelial cells. American Journal of Physiology - Cell Physiology. 2003; 285:C1483-C1493. [PubMed: 12930708]

462. Sabbagh R, Chawla A, Tisdale B, Kwan K, Chatterjee S, Kwiecien J, Kapoor A. Renal histological features according to various warm ischemia time in a porcine laparoscopic and open surgery model. Can Urol Assoc J. 2011; 5:40-43. [PubMed: 21470513]

463. Safirstein R. Gene expression in nephrotoxic and ischemic acute renal failure. Journal of the American Society of Nephrology. 1994; 4:1387-1395. [PubMed: 8161720]

464. Safirstein R. Renal stress response and acute renal failure. Advances in Renal Replacement Therapy. 1997; 4:38-42. [PubMed: 9113239]

465. Safirstein R, Miller P, Dikman S, Lyman N, Shapiro C. Cisplatin nephrotocxicity in rats: defect in papillary hypertonicity. Am J Phyiol. 1981; 241 (Renal Fluid Electrolyte Physiol 10):F175-F185.

466. Safirstein R, Winston J, Moel D, Dikman S, Guttenplan J. Cisplatin nephrotoxicity: insights into mechanism. International Journal of Andrology. 1987; 10:325-346. [PubMed: 3583421]

467. Safirstein R, Zelent AZ, Price PM. Reduced renal prepro-epidermal growth factor mRNA and decreased EGF excretion in ARF. Kidney Int. 1989; 36:810-815. [PubMed: 2615190]

468. Safirstein RL. Cell cycle events during renal injury. Renal Failure. 1999; 21:427-431. [PubMed: 10416223]

469. Saikumar P, Dong Z, Patel Y, Hall K, Hopfer U, Weinberg JM, Venkatachalam MA. Role of hypoxia-induced Bax translocation and cytochrome $\mathrm{c}$ release in reoxygenation injury. Oncogene. 1998; 17:3401-3415. [PubMed: 10030664]

470. Sakai M, Zhang M, Homma T, Garrick B, Abraham JA, McKanna JA, Harris RC. Production of heparin binding epidermal growth factor-like growth factor in the early phase of regeneration after acute renal injury. Isolation and localization of bioactive molecules. The Journal of Clinical Investigation. 1997; 99:2128-2138. [PubMed: 9151785]

471. Salahudeen AK, Huang H, Joshi M, Moore NA, Jenkins JK. Involvement of the Mitochondrial Pathway in Cold Storage and Rewarming-Associated Apoptosis of Human Renal Proximal Tubular Cells. American Journal of Transplantation. 2003; 3:273-280. [PubMed: 12614281]

472. Salman IM, Ameer OZ, Sattar MA, Abdullah NA, Yam MF, Najim HS, Khan AH, Johns EJ. Role of the renal sympathetic nervous system in mediating renal ischaemic injury-induced reductions in renal haemodynamic and excretory functions. Pathology. 42:259-266. [PubMed: 20350220] 
473. Salmela K, Wramner L, Ekberg H, Hauser I, Bentdal O, Lins L-E, Isoniemi H, Bäckman L, Persson N, Neumayer H-H, Jørgensen PIF, Spieker C, Hendry B, Nicholls A, Kirste Gn, Hasche G. A RANDOMIZED MULTICENTER TRIAL OF THE ANTI-ICAM-1 MONOCLONAL ANTIBODY (ENLIMOMAB) FOR THE PREVENTION OF ACUTE REJECTION AND DELAYED ONSET OF GRAFT FUNCTION IN CADAVERIC RENAL TRANSPLANTATION: A Report Of The European Anti-ICAM-1 Renal Transplant Study Group. Transplantation. 1999; 67:729-736. [PubMed: 10096530]

474. Salvioli S, Ardizzoni A, Franceschi C, Cossarizza A. JC-1, but not DiOC6(3) or rhodamine 123, is a reliable fluorescent probe to assess [Delta][Psi] changes in intact cells: implications for studies on mitochondrial functionality during apoptosis. FEBS Letters. 1997; 411:77-82. [PubMed: 9247146]

475. Sanz AB, Santamaria B, Ruiz-Ortega M, Egido J, Ortiz A. Mechanisms of Renal Apoptosis in Health and Disease. J Am Soc Nephrol. 2008; 19:1634-1642. [PubMed: 18632846]

476. Schaudies RP, Johnson JP. Increased soluble EGF after ischemia is accompanied by a decrease in membrane associated precursors. American Journal of Physiology. 1993; 264(Renal Fluid Electrolyte Physiol 33):F523-F531. [PubMed: 8456964]

477. Schiffl H. Renal recovery from acute tubular necrosis requiring renal replacement therapy: a prospective study in critically ill patients. Nephrol Dial Transplant. 2006; 21:1248-1252. [PubMed: 16449291]

478. Schinzel AC, Takeuchi O, Huang Z, Fisher JK, Zhou Z, Rubens J, Hetz C, Danial NN, Moskowitz MA, Korsmeyer SJ. Cyclophilin D is a component of mitochondrial permeability transition and mediates neuronal cell death after focal cerebral ischemia. Proceedings of the National Academy of Sciences of the United States of America. 2005; 102:12005-12010. [PubMed: 16103352]

479. Schmitt R, Cantley LG. The impact of aging on kidney repair. Am J Physiol Renal Physiol. 2008; 294:F1265-F1272. [PubMed: 18287400]

480. Schmitt R, Coca S, Kanbay M, Tinetti ME, Cantley LG, Parikh CR. Recovery of Kidney Function After Acute Kidney Injury in the Elderly: A Systematic Review and Metaanalysis. American Journal of Kidney Diseases. 2008; 52:262-271. [PubMed: 18511164]

481. Schmitt R, Marlier A, Cantley LG. Zag Expression during Aging Suppresses Proliferation after Kidney Injury. Journal of the American Society of Nephrology. 2008; 19:2375-2383. [PubMed: 18815245]

482. Schneider J, Friderichs E, Giertz H. Comparison of the protective effects by human and bovine superoxide dismutase against ischemia- and reperfusion-induced impairment of kidney function in anesthetized rats. Free Radical Biology and Medicine. 1987; 3:21-26. [PubMed: 3623186]

483. Schneider R, Raff U, Vornberger N, Schmidt M, Freund R, Reber M, Schramm L, Gambaryan S, Wanner C, Schmidt HH, Galle J. L-Arginine counteracts nitric oxide deficiency and improves the recovery phase of ischemic acute renal failure in rats. Kidney Int. 2003; 64:216-225. [PubMed: 12787412]

484. Schober A, Muller E, Thurau K, Beck F. The response of heat shock proteins- 25 and 72 to ischaemia in different kidney zones. Pflugers Archiv - European Journal of Physiology. 1997:292-299. [PubMed: 9178629]

485. Schramm L, La M, Heidbreder E, Hecker M, Beckman JS, Lopau K, Zimmermann J, Rendl J, Reiners C, Winderl S, Wanner C, Schmidt HH. L-arginine deficiency and supplementation in experimental acute renal failure and in human kidney transplantation. Kidney Int. 2002; 61:1423-1432. Apr., 2002. [PubMed: 11918749]

486. Schrier RW, Arnold PE, Van Putten VJ, Burke TJ. Cellular calcium in ischemic acute renal failure: Role of calcium entry blockers. Kidney Int. 1987; 32:313-321. [PubMed: 3312759]

487. Schrier RW, Burke TJ. Role of calcium-channel blockers in preventing acute and chronic renal failure. J Cardiovasc Pharmacol. 1991; 18:S38-S43. [PubMed: 1725916]

488. Schulman G, Fogo A, Gung A, Badr K, Hakim R. Complement activation retards resolution of acute ischemic renal failure in the rat. Kidney Int. 1991; 40:1069-1074. [PubMed: 1762308]

489. Schumer M, Colombel MC, Sawczuk IS, Gobe G, Connor J, O'Toole KM, Olsson CA, Wise GJ, Buttyan R. Morphologic, biochemical, and molecular evidence of apoptosis during the 
reperfusion phase after brief periods of renal ischemia. Am J Pathol. 1992; 140:831-838. [PubMed: 1562048]

490. Schwartz N, Hosford M, Sandoval RM, Wagner MC, Atkinson SJ, Bamburg J, Molitoris BA. Ischemia activates actin depolymerizing factor: role in proximal tubule microvillar actin alterations. American Journal of Physiology. 1999; 276:F544-F551. [PubMed: 10198413]

491. Shanley PF, Brezis M, Spokes K, Silva P, Epstein FH, Rosen S. Hypoxic injury in the proximal tubule of the isolated perfused rat kidney. Kidney Int. 1986; 29:1021-1032. [PubMed: 3723924]

492. Sharfuddin AA, Sandoval RM, Berg DT, McDougal GE, Campos SB, Phillips CL, Jones BE, Gupta A, Grinnell BW, Molitoris BA. Soluble Thrombomodulin Protects Ischemic Kidneys. Journal of the American Society of Nephrology. 2009; 20:524-534. [PubMed: 19176699]

493. Sheikh-Hamad D, Cacini W, Buckley A, Isaac J, Truong L, Tsao C, Kishore B. Cellular and molecular studies on cisplatin-induced apoptotic cell death in rat kidney. Archives of Toxicology. 2004; 78:147-155. [PubMed: 14551673]

494. Shelden E, MJ B, FM P, Bonham R. Heat shock protein 27 associates with basolateral cell boudaries in heat shocked and ATP depleted epithelial cells. J Am Soc Nephrol. 2002; 13:332341. [PubMed: 11805160]

495. Sheridan AM, Bonventre JV. Cell biology and molecular mechanisms of injury in ischemic acute renal failure. Current Opinion in Nephrology \& Hypertension. 2000; 9:427-434. [PubMed: 10926180]

496. Shi H, Patschan D, Epstein T, Goligorsky MS, Winaver J. Delayed recovery of renal regional blood flow in diabetic mice subjected to acute ischemic kidney injury. American Journal of Physiology - Renal Physiology. 2007; 293:F1512-F1517. [PubMed: 17881464]

497. Shimizu A, Yamanaka N. Apoptosis and cell desquamation in repair process of ischemic tubular necrosis. Virchows Archives B Cell Pathology. 1993; 64:171-180.

498. Shimizu H, Takahashi T, Suzuki T, Yamasaki A, Fujiwara T, Odaka Y, Hirakawa M, Fujita H, Akagi R. Protective effect of heme oxygenase induction in ischemic acute renal failure. Critical Care Medicine. 2000; 28:809-817. [PubMed: 10752834]

499. Shoag J, Arany Z. Regulation of Hypoxia-Inducible Genes by PGC-1 \{alpha\}. Arterioscler Thromb Vasc Biol. 30:662-666. [PubMed: 19948845]

500. Siegel N, Feldman R, Lytton B, Hayslett J, Kashgarian M. Renal cortical blood flow distribution in obstructive nephropathy in rats. Circulation Research. 1977; 40:379-384. [PubMed: 844151]

501. Siegel NJ, Avison MJ, Reilly HF, Alger JR, Shulman RG. Enhanced recovery of renal ATP with postischemic infusion of ATP-MgCl2 determined by 31P-NMR. American Journal of Physiology. 1983; 245:F530-F534. [PubMed: 6605093]

502. Siegel NJ, Devarajan P, Van Why S. Renal cell injury: metabolic and structural alterations. Pediatric Research. 1994; 36:129-136. [PubMed: 7970927]

503. Siegel NJ, Glazier WB, Chaudry IH, Gaudio KM, Lytton B, Baue AE, Kashgarian M. Enhanced recovery from acute renal failure by the postischemic infusin of adenine nucleotides and magnesium chloride in rats. Kidney Int. 1980; 17:338-349. [PubMed: 7401453]

504. Siew ED, Ware LB, Ikizler TA. Biological Markers of Acute Kidney Injury. Journal of the American Society of Nephrology. 2011; 22:810-820. [PubMed: 21493774]

505. Sikorski EM, Hock T, Hill-Kapturczak N, Agarwal A. The story so far: molecular regulation of the heme oxygenase-1 gene in renal injury. American Journal of Physiology - Renal Physiology. 2004; 286:F425-F441. [PubMed: 14761930]

506. Simmons CF, Bogusky RT, Humes HD. Inhibitory effects of gentamicin on renal mitochondrial oxidative phosphorylation. Journal of Pharmacology and Experimental Therapeutics. 1980; 214:709-715. [PubMed: 7400973]

507. Singh AB, Kaushal V, Megyesi JK, Shah SV, Kaushal GP. Cloning and expression of rat caspase-6 and its localization in renal ischemia/reperfusion injury. Kidney Int. 2002; 62:106-115. [PubMed: 12081569]

508. Singh I, Gulati S, Orak JK, Singh AK. Expression of antioxidant enzymes in rat kidney during ischemia-reperfusion injury. Molecular and Cellular Biochemistry. 1993; 125:97-104. [PubMed: 8283974] 
509. Smoyer WE, Ransom R, Harris RC, Welsh MJ, Lutsch G, Benndorf R. Ischemic acute renal failure induces differential expression of small heat shock proteins. Journal of the American Society of Nephrology. 2000; 11:211-221. [PubMed: 10665928]

510. Snoeijs MG, Vink H, Voesten N, Christiaans MH, Daemen J-WH, Peppelenbosch AG, Tordoir JH, Peutz-Kootstra CJ, Buurman WA, Schurink GWH, van Heurn LWE. Acute ischemic injury to the renal microvasculature in human kidney transplantation. American Journal of Physiology Renal Physiology. 2010; 299:F1134-F1140. [PubMed: 20810613]

511. Solez, K. Acute renal failure. In: Heptinstall, R., editor. Pathophysiology of the kidney. Toronto: Little, Brown and Company; 1983.

512. Solez K, Ideura T, Silvia CB, Hamilton B, Saito H. Clonidine after renal ischemia to lessen acute renal failure and microvascular damage. Kidney Int. 1980; 18:309-322. [PubMed: 7463946]

513. Solez K, Kramer E, Fox J, Heptinstall R. Medullary plasma flow and intravascular leukocyte accumulation in acute renal failure. Kidney Int. 1974; 6:24-37. [PubMed: 4421857]

514. Solez K, Morel-Maroger L, Sraer JD. The morphology of "acute tubular necrosis" in man: analysis of 57 renal biopsies and a comparison with the glycerol model. Medicine (Baltimore). 1979; 58:362-376. [PubMed: 481195]

515. Solez K, Racusen LC, Marcussen N, Slatnik I, Keown P, Burdick JF, Olsen S. Morphology of ischemic acute renal failure, normal function, and cyclosporine toxicity in cyclosporine-treated renal allograft recipients. Kidney Int. 1993; 43:1058-1067. [PubMed: 8510383]

516. Solez K, Racusen LC, Whelton A. Glomerular epithelial cell changes in early postischemic acute renal failure in rabbits and man. Am J Pathol. 1981; 103:163-173. [PubMed: 7015870]

517. Son D, Kojima I, Inagi R, Matsumoto M, Fujita T, Nangaku M. Chronic hypoxia aggravates renal injury via suppression of $\mathrm{Cu} / \mathrm{Zn}-\mathrm{SOD}$ : a proteomic analysis. American Journal of Physiology Renal Physiology. 2008; 294:F62-F72. [PubMed: 17959751]

518. Spargias K, Adreanides E, Demerouti E, Gkouziouta A, Manginas A, Pavlides G, Voudris V, Cokkinos DV. Iloprost Prevents Contrast-Induced Nephropathy in Patients With Renal Dysfunction Undergoing Coronary Angiography or Intervention. Circulation. 2009; 120:17931799. [PubMed: 19841299]

519. Spurgeon-Pechman KR, Donohoe DL, Mattson DL, Lund H, James L, Basile DP. Recovery from acute renal failure predisposes hypertension and secondary renal disease in response to elevated sodium. Am J Physiol Renal Physiol. 2007; 293:F269-F278. [PubMed: 17507599]

520. Spurgeon KS, Donohoe DL, Basile DP. Transforming growth factor-s in acute renal failure: receptor expression, influence in cell proliferation, cellularity and vascularization after recovery from injury. Am J Physiol Renal Physiol. 2005; 288:F568-F577. [PubMed: 15536165]

521. Sreedharan, R.; Devarajan, P.; Van Why, S. Pathogenesis of acute renal failure. In: Avner, E.; Harmon, W.; Niaudet, P.; Yoshikawa, N., editors. Pediatric Nephrology. Heidleberg: SpringerVerlag; 2009. p. 1579-1602.

522. Star RA. Treatment of acute renal failure. Kidney Int. 1998; 54:1817-1831. [PubMed: 9853246]

523. Stracke S, Ernst F, Jehle DR, Grunewald RW, Haller H, Keller F, Jehle PM. Differentiating and proliferative effects of HGF in renal proximal tubular cells are mediated via different signalling pathways. Nephrology Dialysis Transplantation. 1998; 13:1398-1405.

524. Stromski ME, Cooper K, Thulin G, Gaudio KM, Siegel NJ, Shulman RG. Chemical and functional correlates of postischemic renal ATP levels. Proceedings of the National Academy of Sciences of the United States of America. 1986; 83:6142-6145. [PubMed: 3461481]

525. Sucher R, Gehwolf P, Oberhuber R, Hermann M, Margreiter C, Werner ER, Obrist P, Schneeberger S, Ollinger R, Margreiter R, Brandacher G. Tetrahydrobiopterin protects the kidney from ischemia-reperfusion injury. Kidney Int. 2010; 77:681-689. [PubMed: 20164829]

526. Sugiura H, Yoshida T, Tsuchiya K, Mitobe M, Nishimura S, Shirota S, Akiba T, Nihei H. Klotho reduces apoptosis in experimental ischaemic acute renal failure. Nephrology Dialysis Transplantation. 2005; 20:2636-2645.

527. Sugiura T, Kobuchi S, Tsutsui H, Takaoka M, Fujii T, Hayashi K, Matsumura Y. Preventive mechanisms of agmatine against ischemic acute kidney injury in rats. European Journal of Pharmacology. 2009; 603:108-113. [PubMed: 19105953] 
528. Sun D, Samuelson LC, Yang T, Huang Y, Paliege A, Saunders T, Briggs J, Schnermann J. Mediation of tubuloglomerular feedback by adenosine: Evidence from mice lacking adenosine 1 receptors. Proceedings of the National Academy of Sciences of the United States of America. 2001; 98:9983-9988. [PubMed: 11504952]

529. Supavekin S, Zhang W, Kucherlapati R, Kaskel FJ, Moore LC, Devarajan P. Differential gene expression following early renal ischemia/reperfusion. Kidney Int. 2003; 63:1714-1724. [PubMed: 12675847]

530. Surendran K, Kopan R. Chromatin-based Mechanisms of Renal Epithelial Differentiation. Journal of the American Society of Nephrology. 2011; 22:1208-1212. [PubMed: 21700830]

531. Sutton TA. Alteration of microvascular permeability in acute kidney injury. Microvascular Research. 2009; 77:4-7. [PubMed: 18938184]

532. Sutton TA, Fisher CJ, Molitoris BA. Microvascular endothelial injury and dysfunction during ischemic acute renal failure. Kidney Int. 2002; 62:1539-1549. [PubMed: 12371954]

533. Sutton TA, Kelly KJ, Mang H, Plotkin Z, Sandoval R, Dagher PC. Minocycline reduces microvascular leakage in a model of ischemic renal injury. Am J Physiol Renal Physiol. 2005; 288:F91-F97. [PubMed: 15353401]

534. Sutton TA, Mang HE, Campos SB, Sandoval RM, Yoder MC, Molitoris BA. Injury of the renal microvascular endothelium alters barrier function after ischemia. American Journal of Physiology - Renal Physiology. 2003; 285:F191-F198. [PubMed: 12684225]

535. Suzuki S, Maruyama S, Sato W, Morita Y, Sato F, Miki Y, Kato S, Katsuno M, Sobue G, Yuzawa Y, Matsuo S. Geranylgeranylacetone ameliorates ischemic acute renal failure via induction of Hsp70. 2005; 67:2210-2220.

536. Tadagavadi RK, Reeves WB. Renal Dendritic Cells Ameliorate Nephrotoxic Acute Kidney Injury. Journal of the American Society of Nephrology. 2010; 21:53-63. [PubMed: 19875815]

537. Takada M, Chandraker A, Nadeau KC, Sayegh MH, Tilney NL. The role of the B7 costimulatory pathway in experimental cold ischemia/reperfusion injury. The Journal of Clinical Investigation. 1997; 100:1199-1203. [PubMed: 9276737]

538. Takeyama N, Miki S, Hirakawa A, Tanaka T. Role of the Mitochondrial Permeability Transition and Cytochrome c Release in Hydrogen Peroxide-Induced Apoptosis. Experimental Cell Research. 2002; 274:16-24. [PubMed: 11855853]

539. Taman M, Liu Y, Tolbert E, Dworkin L. Increase urinary hepatocyte growth factor excretion in human acute renal failure. Clin Nephrol. 1997; 48:241-245. [PubMed: 9352159]

540. Terada Y, Tanaka H, Okado T, Shimamura H, Inoshita S, Kuwahara M, Sasaki S. Expression and Function of the Developmental Gene Wnt-4 during Experimental Acute Renal Failure in Rats. Journal of the American Society of Nephrology. 2003; 14:1223-1233. [PubMed: 12707392]

541. Terry B, Jones D, Mueller C. Experimental ischemic renal arterial necrosis with resolution. American Journal of Pathology. 1970; 58:69-83. [PubMed: 5414018]

542. Thadhani R, Pascual M, Bonventre JV. Acute Renal Failure. New England Journal of Medicine. 1996; 334:1448-1460. [PubMed: 8618585]

543. Thakar CV, Quate-Operacz M, Leonard AC, Eckman MH. Outcomes of Hemodialysis Patients in a Long-term Care Hospital Setting: A Single-Center Study. American Journal of Kidney Diseases. 2010; 55:300-306. [PubMed: 20006413]

544. Thomas SE, Anderson S, Gordon KL, Oyama TT, Shankland SJ, Johnson RJ. Tubulointerstitial disease in aging: evidence for underlying peritubular capillary damage, a potential role for renal ischemia. Journal of the American Society of Nephrology. 1998; 9:231-242. [PubMed: 9527399]

545. Thurman JM, Lenderink AM, Royer PA, Coleman KE, Zhou J, Lambris JD, Nemenoff RA, Quigg RJ, Holers VM. C3a Is Required for the Production of CXC Chemokines by Tubular Epithelial Cells after Renal Ishemia/Reperfusion. The Journal of Immunology. 2007; 178:1819_ 1828. [PubMed: 17237432]

546. Thurman JM, Ljubanovic D, Edelstein CL, Gilkeson GS, Holers VM. Lack of a Functional Alternative Complement Pathway Ameliorates Ischemic Acute Renal Failure in Mice. The Journal of Immunology. 2003; 170:1517-1523. [PubMed: 12538716]

547. Thurman JM, Ljubanovic D, Royer PA, Kraus DM, Molina H, Barry NP, Proctor G, Levi M, Holers VM. Altered renal tubular expression of the complement inhibitor Crry permits 
complement activation after ischemia/reperfusion. The Journal of Clinical Investigation. 2006; 116:357-368. [PubMed: 16444293]

548. Tobimatsu M, Euda Y, Saito S, Tsumagari T, Konomi K. Effects of a stable prostocyclin analog on experimental ischemic acute renal failure. Ann Surg. 1988; 208:65-70. [PubMed: 3291800]

549. Togel F, Isaac J, Hu Z, Weiss K, Westenfelder C. Renal SDF-1 signals mobilization and homing of CXCR4-positive cells to the kidney after ischemic injury. Kidney Int. 2005; 67:1772-1784. [PubMed: 15840024]

550. Togel F, Isaac J, Westenfelder C. Hematopoietic Stem Cell Mobilization-Associated Granulocytosis Severely Worsens Acute Renal Failure. Journal of the American Society of Nephrology. 2004; 15:1261-1267. [PubMed: 15100366]

551. Togel F, Weiss K, Yang Y, Hu Z, Zhang P, Westenfelder C. Vasculotropic, paracrine actions of infused mesenchymal cells are important to the recovery from acute kidney injury. Am J Physiol. 2007 epub;

552. Togel F, Yang Y, Zhang P, Hu Z, Westenfelder C. Bioluminescence imaging to monitor the in vivo distribution of administered mesenchymal stem cells in acute kidney injury. American Journal of Physiology - Renal Physiology. 2008; 295:F315-F321. [PubMed: 18480180]

553. Togel FE, Westenfelder C. Mesenchymal stem cells: a new therapeutic tool for AKI. Nat Rev Nephrol. 2010; 6:179-183. [PubMed: 20186233]

554. Toronyi E, Lord R, Bowen ID, Perner F, Szende B. RENAL TUBULAR CELL NECROSIS AND APOPTOSIS IN TRANSPLANTED KIDNEYS. Cell Biology International. 2001; 25:267270. [PubMed: 11352501]

555. Torras J, Herrero-Fresneda I, Lloberas N, Riera M, Ma Cruzado J, Ma Grinyo J. Promising effects of ischemic preconditioning in renal transplantation. Kidney International. 2002; 61:2218-2227. [PubMed: 12028463]

556. Torras J, Seron D, Herrero I, Martinez-Castelao A, Carrera M, Alsina J, Grino JM. Renal protective effect of liposomed superoxide dismutase in an experimental warm ischemia model. Transplant International. 1994; 7:S472-S475. [PubMed: 11271283]

557. Traktuev DO, Merfeld-Clauss S, Li J, Kolonin M, Arap W, Pasqualini R, Johnstone BH, March KL. A Population of Multipotent CD34-Positive Adipose Stromal Cells Share Pericyte and Mesenchymal Surface Markers, Reside in a Periendothelial Location, and Stabilize Endothelial Networks. Circ Res. 2008; 102:77-85. [PubMed: 17967785]

558. Tsukamoto T, Nigam SK. Tight Junction Proteins Form Large Complexes and Associate with the Cytoskeleton in an ATP Depletion Model for Reversible Junction Assembly. Journal of Biological Chemistry. 1997; 272:16133-16139. [PubMed: 9195909]

559. Tsuruta F, Sunayama J, Mori Y, Hattori S, Shimizu S, Tsujimoto Y, Yoshioka K, Masuyama N, Gotoh Y. JNK promotes Bax translocation to mitochondria through phosphorylation of 14-3-3 proteins. Embo J. 2004; 23:1889-1899. [PubMed: 15071501]

560. Tumlin JA, Finkel KW, Murray PT, Samuels J, Cotsonis G, Shaw AD. Fenoldopam Mesylate in Early Acute Tubular Necrosis: A Randomized, Double-Blind, Placebo-Controlled Clinical Trial. American journal of kidney diseases : the official journal of the National Kidney Foundation. 2005; 46:26-34. [PubMed: 15983954]

561. Uchida S, Endou H. Substrate specificity to maintain cellular ATP along the mouse nephron. Am J Physiol. 1988; 255:F977-F983. [PubMed: 2847554]

562. Uchino S, Kellum JA, Bellomo R, Doig GS, Morimatsu H, Morgera S, Schetz M, Tan I, Bouman C, Macedo E, Gibney N, Tolwani A, Ronco C. Acute renal failure in critically ill patients: a multinational, multicenter study. JAMA. 2005; 294:813-818. [PubMed: 16106006]

563. Ueda N, Kaushal GP, Shah SV. Apoptotic mechanisms in acute renal failure. American Journal of Medicine. 2000; 108:403-415. [PubMed: 10759097]

564. Ueda N, Shah SV. Tubular cell damage in acute renal failure-apoptosis, necrosis, or both. Nephrology Dialysis Transplantation. 2000; 15:318-323.

565. Urakawa I, Yamazaki Y, Shimada T, Iijima K, Hasegawa H, Okawa K, Fujita T, Fukumoto S, Yamashita T. Klotho converts canonical FGF receptor into a specific receptor for FGF23. Nature. 2006; 444:770-774. [PubMed: 17086194] 
566. Van Arsdale R. THE PHARMACOLOGY OF MERCURY. Journal of the American Medical Association. 1923; 81:1748-1752.

567. Van Why S, Mann A, Ardito T, Thulin G, Ferris S, MacLeod M, Kashgarian M, Siegel NJ. Hsp27 associates with actin and limits injury in energy depleted renal epithelia. J Am Soc Nephrol. 2003; 13:98-106. [PubMed: 12506142]

568. Van Why SK, Hildebrandt F, Ardito T, Mann AS, Siegel NJ, Kashgarian M. Induction and intracellular localization of HSP-72 after renal ischemia. American Journal of Physiology. 1992; 263:F769-F775. [PubMed: 1443167]

569. Van Why SK, Mann AS, Ardito T, Siegel NJ, Kashgarian M. Expression and molecular regulation of $\mathrm{Na}(+)-\mathrm{K}(+)$-ATPase after renal ischemia. American Journal of Physiology. 1994; 267:F75-F85. [PubMed: 8048568]

570. Van Why SK, Siegel NJ. Heat shock proteins in renal injury and recovery. Current Opinion in Nephrology \& Hypertension. 1998; 7:407-412. [PubMed: 9690040]

571. Van Why, SK.; Siegel, NJ. Heat shock proteins: Role in prevention and recovery from acute renal failure. In: Molitoris, BA.; Finn, WF., editors. Acute renal failure: A companion to Brenner and Rectors The Kidney. Philadelphia: WB Sanders; 2001. p. 143-156.

572. Vásárhelyi B, Tóth-Heyn P, Treszl A, Tulassay T. Genetic polymorphisms and risk for acute renal failure in preterm neonates. Pediatric Nephrology. 2005; 20:132-135. [PubMed: 15627170]

573. Venkatachalam MA, Bernard DB, Donohoe JF, Levinsky N. Ischemic damage and repair in the rat proximal tubule: differences among S1, S2 and S3 segments. Kidney Int. 1978; 14:31-49. [PubMed: 682423]

574. Venkatachalam MA, Griffin KA, Lan R, Geng H, Saikumar P, Bidani AK. Acute kidney injury: a springboard for progression in chronic kidney disease. American Journal of Physiology - Renal Physiology. 2010; 298:F1078-F1094. [PubMed: 20200097]

575. Vera T, Henegar JR, Drummond HA, Rimoldi JM, Stec DE. Protective Effect of Carbon Monoxide-Releasing Compounds in Ischemia-Induced Acute Renal Failure. Journal of the American Society of Nephrology. 2005; 16:950-958. [PubMed: 15728782]

576. Villanueva S, Cespedes C, Gonzalez AA, Roessler E, Vio CP. Inhibition of bFGF-receptor type 2 increases kidney damage and suppresses nephrogenic protein expression after ischemic acute renal failure. American Journal of Physiology - Regulatory, Integrative and Comparative Physiology. 2008; 294:R819-R828.

577. Vinuesa E, Hotter G, Jung M, Herrero-Fresneda I, Torras J, Sola A. Macrophage involvement in the kidney repair phase after ischaemia/reperfusion injury. The Journal of Pathology. 2008; 214:104-113. [PubMed: 17973244]

578. Vogt M, Farber JL. On the molecular pathology of ischemic renal cell death: Reversible and irreversible cellular and mitochondrial metabolic alterations. American Journal of Pathology. 1968; 53

579. Vukicevic S, Basic V, Rogic D, Basic N, Shih MS, Shepard A, Jin D, Dattatreyamurty B, Jones W, Dorai H, Ryan S, Griffiths D, Maliakal J, Jelic M, Pastorcic M, Stavljenic A, Sampath TK. Osteogenic protein-1 (bone morphogenetic protein-7) reduces severity of injury after ischemic acute renal failure in rat. The Journal of Clinical Investigation. 1998; 102:202-214. [PubMed: 9649574]

580. Wada T, Sakai N, Matsushima K, Kaneko S. Fibrocytes: A new insight into kidney fibrosis. Kidney Int. 2007; 72:269-273. [PubMed: 17495856]

581. Wahlberg JAN, Karlberg L, Persson AEG. Total and regional renal blood flow during complete unilateral ureteral obstruction. Acta Physiologica Scandinavica. 1984; 121:111-118. [PubMed: 6475542]

582. Waikar SS, Curhan GC, Wald R, McCarthy EP, Chertow GM. Declining mortality in patients with acute renal failure, 1988 to 2002. J Am Soc Nephrol. 2006; 17:1143-1150. [PubMed: 16495376]

583. Waikar SS, Winkelmayer WC. Chronic on acute renal failure: long-term implications of severe acute kidney injury. JAMA. 2009; 302:1227-1229. [PubMed: 19755705] 
584. Wald R, Quinn RR, Luo J, Li P, Scales DC, Mamdani MM, Ray JG. Chronic dialysis and death among survivors of acute kidney injury requiring dialysis. JAMA. 2009; 302:1179-1185. [PubMed: 19755696]

585. Walker PD. Alterations in renal tubular extracellular matrix components after ischemiareperfusion injury to the kidney. Laboratory Investigation. 1994; 70:339-346. [PubMed: 8145528]

586. Wang A, Holcslaw T, Bashore TM, Freed MI, Miller D, Rudnick MR, Szerlip H, Thames MD, Davidson CJ, Shusterman N, Schwab SJ. Exacerbation of radiocontrast nephrotoxicity by endothelin receptor antagonism. Kidney Int. 2000; 57:1675-1680. [PubMed: 10760103]

587. Wang J, Wei Q, Wang C-Y, Hill WD, Hess DC, Dong Z. Minocycline Upregulates Bcl-2 and Protects against Cell Death in Mitochondria. Journal of Biological Chemistry. 2004; 279:1994819954. [PubMed: 15004018]

588. Wang W, Falk SA, Jittikanont S, Gengaro PE, Edelstein CL, Schrier RW. Protective effect of renal denervation on normotensive endotoxemia-induced acute renal failure in mice. American Journal of Physiology - Renal Physiology. 2002; 283:F583-F587. [PubMed: 12167611]

589. Wang W, Mitra A, Poole B, Falk S, Lucia MS, Tayal S, Schrier R. Endothelial nitric oxide synthase-deficient mice exhibit increased susceptibility to endotoxin-induced acute renal failure. American Journal of Physiology - Renal Physiology. 2004; 287:F1044-F1048. [PubMed: 15475535]

590. Wang W, Zolty E, Falk S, Summer S, Stearman R, Geraci M, Schrier R. Prostacyclin in endotoxemia-induced acute kidney injury: cyclooxygenase inhibition and renal prostacyclin synthase transgenic mice. American Journal of Physiology - Renal Physiology. 2007; 293:F1131-F1136. [PubMed: 17652370]

591. Wang Y, Harris DCH. Macrophages in Renal Disease. Journal of the American Society of Nephrology. 2011; 22:21-27. [PubMed: 21209251]

592. Wang Y, John R, Chen J, Richardson JA, Shelton JM, Bennett M, Zhou XJ, Nagami GT, Zhang Y, Wu QQ, Lu CY. IRF-1 Promotes Inflammation Early after Ischemic Acute Kidney Injury. Journal of the American Society of Nephrology. 2009; 20:1544-1555. [PubMed: 19443641]

593. Wang Y, Wang YP, Zheng G, Lee VWS, Ouyang L, Chang DHH, Mahajan D, Coombs J, Wang YM, Alexander SI, Harris DCH. Ex vivo programmed macrophages ameliorate experimental chronic inflammatory renal disease. Kidney Int. 2007; 72:290-299. [PubMed: 17440493]

594. Wang Z, Chen J-K, Wang S-w, Moeckel G, Harris RC. Importance of Functional EGF Receptors in Recovery from Acute Nephrotoxic Injury. Journal of the American Society of Nephrology. 2003; 14:3147-3154. [PubMed: 14638913]

595. Wei MC, Zong W-X, Cheng EH-Y, Lindsten T, Panoutsakopoulou V, Ross AJ, Roth KA, MacGregor GR, Thompson CB, Korsmeyer SJ. Proapoptotic BAX and BAK: A Requisite Gateway to Mitochondrial Dysfunction and Death. Science. 2001; 292:727-730. [PubMed: 11326099]

596. Wei Q, Alam MM, Wang M-H, Yu F, Dong Z. Bid activation in kidney cells following ATP depletion in vitro and ischemia in vivo. Am J Physiol Renal Physiol. 2004; 286:F803-F809. [PubMed: 14678945]

597. Wei Q, Dong G, Franklin J, Dong Z. The pathological role of Bax in cisplatin nephrotoxicity. Kidney Int. 2007; 72:53-62. [PubMed: 17410096]

598. Wei Q, Yin X-M, Wang M-H, Dong Z. Bid deficiency ameliorates ischemic renal failure and delays animal death in C57BL/6 mice. Am J Physiol Renal Physiol. 2006; 290:F35-F42. [PubMed: 16106037]

599. Weinberg JM. The cell biology of ischemic renal injury. Kidney International. 1991; 39:476-500. [PubMed: 2062034]

600. Weinberg JM, Davis JA, Abarzua M, Rajan T. Cytoprotective effects of glycine and glutathione against hypoxic injury to renal tubules. Journal of Clinical Investigation. 1987; 80:1446-1454. [PubMed: 2890657]

601. Weinberg JM, Molitoris BA. Illuminating Mitochondrial Function and Dysfunction using Multiphoton Technology. J Am Soc Nephrol. 2009; 20:1164-1166. [PubMed: 19470668] 
602. Weinberg JM, Venkatachalam MA, Roeser NF, Nissim I. Mitochondrial dysfunction during hypoxia/reoxygenation and its correction by anaerobic metabolism of citric acid cycle intermediates. Proceedings of the National Academy of Sciences of the United States of America. 2000; 97:2826-2831. [PubMed: 10717001]

603. Wesche-Soldato DE, Chung C-S, Lomas-Neira J, Doughty LA, Gregory SH, Ayala A. In vivo delivery of caspase-8 or Fas siRNA improves the survival of septic mice. Blood. 2005; 106:2295-2301. [PubMed: 15941915]

604. Westhoff JH, Schildhorn C, Jacobi C, Homme M, Hartner A, Braun H, Kryzer C, Wang C, von Zglinicki T, Kranzlin B, Gretz N, Melk A. Telomere Shortening Reduces Regenerative Capacity after Acute Kidney Injury. Journal of the American Society of Nephrology. 2009

605. Wilhelm SM, Simonson MS, Robinson AV, Stowe NT, Schulak JA. Endothelin up-regulation and localization following renal ischemia and reperfusion. Kidney International. 1999; 55:10111018. [PubMed: 10027938]

606. Willinger C, Schramek H, Pfaller K, Pfaller W. Tissue distribution of Neutrophils in postischemic acute renal failure. Virchows Archiv B Cell Pathology Zell-pathologie. 1992; 62:237-243.

607. Wilson PD, Schrier RW. Nephron segment and calcium as determinants of anoxic cell death in renal cultures. Kidney Int. 1986; 29:1172-1179. [PubMed: 3747334]

608. Witzgall R, Brown D, Schwarz C, Bonventre JV. Localization of proliferating cell nuclear antigen, vimentin, c-fos and clusterin in the post-ischemic kidney. The Journal of Clinical Investigation. 1994; 93:2175-2188. [PubMed: 7910173]

609. Wu H, Chen G, Wyburn KR, Yin J, Bertolino P, Eris JM, Alexander SI, Sharland AF, Chadban SJ. TLR4 activation mediates kidney ischemia/reperfusion injury. The Journal of Clinical Investigation. 2007; 117:2847-2859. [PubMed: 17853945]

610. Yamamoto T, Tada T, Brodsky SV, Tanaka H, Noiri E, Kajiya F, Goligorsky MS. Intravital videomicroscopy of peritubular capillaries in renal ischemia. American Journal of Physiology Renal Fluid \& Electrolyte Physiology. 2002; 282:F1150-F1155.

611. Yamanobe T, Okada F, Iuchi Y, Onuma K, Tomita Y, Fujii J. Deterioration of ischemia/ reperfusion-induced acute renal failure in SOD1-deficient mice. Free Radical Research. 2007; 41:200-207. [PubMed: 17364946]

612. Yamashita J, Kita S, Iwamoto T, Ogata M, Takaoka M, Tazawa N, Nishikawa M, Wakimoto K, Shigekawa M, Komuro I, Matsumura Y. Attenuation of Ischemia/Reperfusion-Induced Renal Injury in Mice Deficient in $\mathrm{Na}+\mathrm{Ca} 2+$ Exchanger. Journal of Pharmacology and Experimental Therapeutics. 2003; 304:284-293. [PubMed: 12490603]

613. Yamasowa H, Shimizu S, Inoue T, Takaoka M, Matsumura Y. Endothelial Nitric Oxide Contributes to the Renal Protective Effects of Ischemic Preconditioning. Journal of Pharmacology and Experimental Therapeutics. 2005; 312:153-159. [PubMed: 15308652]

614. Yanagita M, Okuda T, Endo S, Tanaka M, Takahashi K, Sugiyama F, Kunita S, Takahashi S, Fukatsu A, Yanagisawa M, Kita T, Sakurai T. Uterine sensitizationassociated gene (USAG-1), a novel BMP antagonist expressed in the kidney, accelerates tubular injury. The Journal of Clinical Investigation. 2006; 116:70-79. [PubMed: 16341262]

615. Yang C-C, Lin L-C, Wu M-S, Chien C-T, Lai M-K. Repetitive Hypoxic Preconditioning Attenuates Renal Ischemia/Reperfusion Induced Oxidative Injury via Upregulating HIF-1[alpha]Dependent bcl-2 Signaling. Transplantation. 2009; 88:1251-1260 1210.1097/TP. 1250b1013e3181bb1254a1207. [PubMed: 19996924]

616. Yang CW, Li C, Jung JY, Shin SJ, Choi BS, Lim SW, Sun BK, Kim YS, Kim J, Chang YS, Bang BK. Preconditioning with erythropoietin protects against subsequent ischemia-reperfusion injury in rat kidney. The FASEB Journal.

617. Yang L, Besschetnova TY, Brooks CR, Shah JV, Bonventre JV. Epithelial cell cycle arrest in G2/ M mediates kidney fibrosis after injury. Nat Med. 16:535-543. [PubMed: 20436483]

618. Yano T, Itoh Y, Kawamura E, Maeda A, Egashira N, Nishida M, Kurose H, Oishi R. Amphotericin B-Induced Renal Tubular Cell Injury Is Mediated by Na+ Influx through IonPermeable Pores and Subsequent Activation of Mitogen-Activated Protein Kinases and Elevation of Intracellular Ca2+ Concentration. Antimicrob Agents Chemother. 2009; 53:1420-1426. [PubMed: 19139282] 
619. Yasuda H, Leelahavanichkul A, Tsunoda S, Dear JW, Takahashi Y, Ito S, Hu X, Zhou H, Doi K, Childs R, Klinman DM, Yuen PST, Star RA. Chloroquine and inhibition of Tolllike receptor 9 protect from sepsis-induced acute kidney injury. American Journal of Physiology - Renal Physiology. 2008; 294:F1050-F1058. [PubMed: 18305095]

620. Yehuda Z, Iris G. Antioxidants attenuate endotoxin-induced acute renal failure in rats. American journal of kidney diseases : the official journal of the National Kidney Foundation. 1995; 25:5157. [PubMed: 7810533]

621. Yin M, Wheeler MD, Connor HD, Zhong Z, Bunzendahl H, Dikalova A, Samulski RJ, Schoonhoven R, Mason RP, Swenberg JA, Thurman RG. Cu/Zn-Superoxide Dismutase Gene Attenuates Ischemia-Reperfusion Injury in the Rat Kidney. Journal of the American Society of Nephrology. 2001; 12:2691-2700. [PubMed: 11729238]

622. Yin X, Apostolov EO, Shah SV, Wang X, Bogdanov KV, Buzder T, Stewart AG, Basnakian AG. Induction of Renal Endonuclease G by Cisplatin Is Reduced in DNase IDeficient Mice. J Am Soc Nephrol. 2007; 18:2544-2553. [PubMed: 17675668]

623. Yoder MC. Defining human endothelial progenitor cells. Journal of Thrombosis and Haemostasis. 2009; 7:49-52. [PubMed: 19630767]

624. Yokota N, Daniels F, Crosson J, Rabb H. Protective effect of T cell depletion in murine renal ischemia-reperfusion injury. Transplantation. 2002; 74:759-763. [PubMed: 12364852]

625. Yoshioka T, Fogo A, Beckman JK. Reduced activity of antioxidant enzymes underlies contrast media-induced renal injury in volume depletion. Kidney Int. 1992; 41:1008-1015. [PubMed: 1513081]

626. Ysebaert DK, De Greef KE, De Beuf A, Van Rompay AR, Vercauteren S, Persy VP, De Broe ME. T cells as mediators in renal ischemia/reperfusion injury. Kidney Int. 2004; 66:491-496. [PubMed: 15253695]

627. Ysebaert DK, De Greef KE, Vercauteren SR, Ghielli M, Verpooten GA, Eyskens EJ, De Broe ME. Identification and kinetics of leukocytes after severe ischaemia/reperfusion renal injury. Nephrol Dial Transplant. 2000; 15:1562-1574. [PubMed: 11007823]

628. Ysebaert DK, De Greef KE, Vercauteren SR, Ghielli M, Verpooten GA, Eyskens EJ, De Broe ME. Identification and kinetics of leukocytes after severe ischaemia/reperfusion renal injury. Nephrology Dialysis Transplantation. 2000; 15:1562-1574.

629. Yuan H-T, Li X-Z, Pitera JE, Long DA, Woolf AS. Peritubular Capillary Loss after Mouse Acute Nephrotoxicity Correlates with Down-Regulation of Vascular Endothelial Growth Factor-A and Hypoxia-Inducible Factor-1a. The American journal of pathology. 2003; 163:2289-2301. [PubMed: 14633603]

630. Zager R, Mahan J, Merola A. Effects of mannitol on the postischemic kidney. Biochemica, functinal, and morphologic assessments. Lab Invest. 1985; 53:433-442. [PubMed: 3930877]

631. Zager RA. Mitochondrial free radical production induces lipid peroxidation during myohemoglobinuria. Kidney Int. 1996; 49:741-751. [PubMed: 8648915]

632. Zager RA, Burkhart KM, Johnson ACM, Sacks BM. Increased proximal tubular cholesterol content: Implications for cell injury and [1dquo]acquired cytoresistance[rdquo]. Kidney Int. 1999; 56:1788-1797. [PubMed: 10571787]

633. Zager RA, Foerder C, Bredl C. The influence of mannitol on myoglobinuric acute renal failure: functional, biochemical, and morphological assessments. Journal of the American Society of Nephrology. 1991; 2:848-855. [PubMed: 1751788]

634. Zager RA, Fuerstenberg SM, Baehr PH, Myerson D, Torok-Storb B. An evaluation of antioxidant effects on recovery from postischemic acute renal failure. Journal of the American Society of Nephrology. 1994; 4:1588-1597. [PubMed: 7912960]

635. Zager RA, Johnson A. Renal cortical cholesterol accumulation is an integral component of the systemic stress response. Kidney Int. 2001; 60:2299-2310. [PubMed: 11737603]

636. Zager RA, Jurkowitz MS, Merola AJ. Responses of the normal rat kidney to sequential ischemic events. American Journal of Physiology. 1985; 249:F148-F159. [PubMed: 4014471]

637. Zager RA, Shah V, Shah H, Zager P, Johnson A, Hanson S. The mevalonate pathway during acute tubular injury: Selected determinants and consequences. Am J Pathol. 2002; 161:681-692. [PubMed: 12163393] 
638. Zhang B, Ramesh G, Uematsu S, Akira S, Reeves WB. TLR4 Signaling Mediates Inflammation and Tissue Injury in Nephrotoxicity. Journal of the American Society of Nephrology. 2008; 19:923-932. [PubMed: 18256356]

639. Zhang GH, Ichimura T, Wallin A, Kan M, Stevens JL. Regulation of rat proximal tubule epithelial cell growth by fibroblast growth factors, insulin-like growth factor-1 and transforming growth factor-beta, and analysis of fibroblast growth factors in rat kidney. Journal of Cellular Physiology. 1991; 148:295-305. [PubMed: 1715345]

640. Zhang X, Zheng X, Sun H, Feng B, Chen G, Vladau C, Li M, Chen D, Suzuki M, Min L, Liu W, Garcia B, Zhong R, Min W-P. revention of Renal Ischemic Injury by Silencing the Expression of Renal Caspase 3 and Caspase 8. Transplantation. 2006; 82:1728-1732. 1710.1097/1701.tp. 0000250764.0000217636.ba. [PubMed: 17198267]

641. Zhang Z-X, Wang S, Huang X, Min W-P, Sun H, Liu W, Garcia B, Jevnikar AM. NK Cells Induce Apoptosis in Tubular Epithelial Cells and Contribute to Renal Ischemia-Reperfusion Injury. J Immunol. 2008; 181:7489-7498. [PubMed: 19017938]

642. Zhou H, Kato A, Yasuda H, Odamaki M, Itoh H, Hishida A. The induction of heat shock protein-72 attenuates cisplatin-induced acute renal failure in rats. Pflugers Archiv - European Journal of Physiology. 2003; 446:116-124. [PubMed: 12690470]

643. Zhou W, Farrar CA, Abe K, Pratt JR, Marsh JE, Wang Y, Stahl GL, Sacks SH. Predominant role for C5b-9 in renal ischemia/reperfusion injury. Journal of Clinical Investigation. 2000; 105:1363-1371. [PubMed: 10811844]

644. Zhou Y, Vaidya VS, Brown RP, Zhang J, Rosenzweig BA, Thompson KL, Miller TJ, Bonventre JV, Goering PL. Comparison of Kidney Injury Molecule-1 and Other Nephrotoxicity Biomarkers in Urine and Kidney Following Acute Exposure to Gentamicin, Mercury, and Chromium. Toxicological Sciences. 2008; 101:159-170. [PubMed: 17934191]

645. Zhuang S, Kinsey GR, Rasbach K, Schnellmann RG. Heparin-binding epidermal growth factor and Src family kinases in proliferation of renal epithelial cells. American Journal of Physiology Renal Physiology. 2008; 294:F459-F468. [PubMed: 18171996]

646. Zhuang S, Schnellmann RG. A Death-Promoting Role for Extracellular Signal- Regulated Kinase. Journal of Pharmacology and Experimental Therapeutics. 2006; 319:991-997. [PubMed: 16801453]

647. Zou AP, Li N, Cowley AW Jr. Production and actions of superoxide in the renal medulla. Hypertension. 2001; 37:547-553.. [PubMed: 11230333]

648. Zou AP, Wu F, Cowley AW Jr. Protective effect of angiotensin II-induced increase in nitric oxide in the renal medullary circulation. Hypertension. 1998; 31:271-276. [PubMed: 9453315]

649. Zuk A, Bonventre JV, Brown D, Matlin KS. Polarity, integrin, and extracellular matrix dynamics in the postischemic rat kidney. American Journal of Physiology - Cell Physiology. 1998; 275:C711-C731.

650. Zuk A, Bonventre JV, Matlin KS. Expression of fibronectin splice variants in the postischemic rat kidney. American Journal of Physiology - Renal Fluid \& Electrolyte Physiology. 2001; 280:F1037-F1053. 


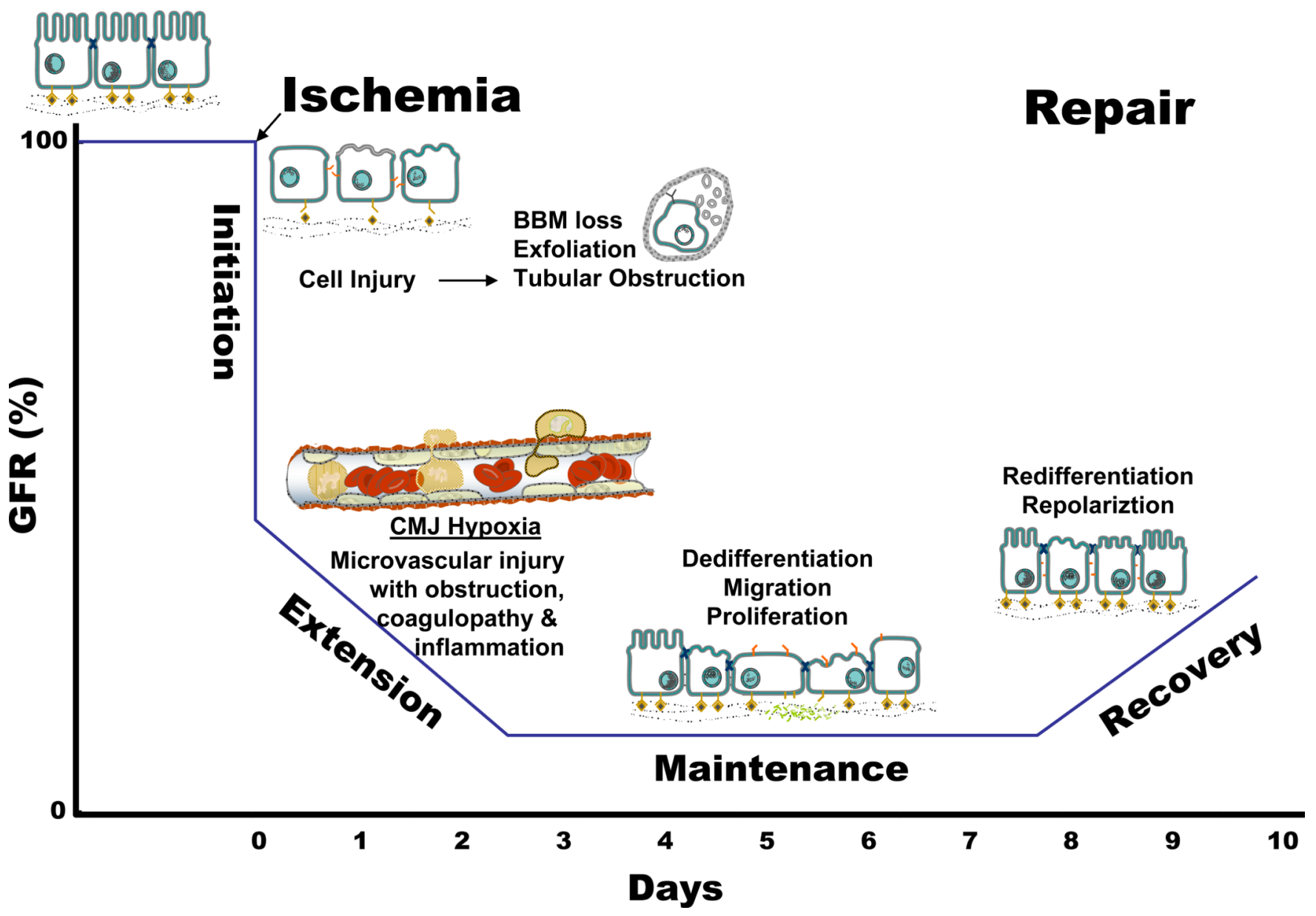

Figure 1. Relationship between the clinical phases and the cellular phases of ischemic acute kidney injury (AKI), and the temporal impact on organ function as represented by glomerular filtration rate (GFR)

Prerenal azotemia exists when a reduction in renal blood flow causes a reduction in GFR. A variety of cellular and vascular adaptations maintain renal epithelial integrity during this phases. The initiation phase ossuces when a further reduction in renal blood flow results in cellular injury, particularly the renal tubular epithelial cells, and a continued decline in GFR. Vascular and inflammatory processes that contribute to further cell injury and a further decline in GFR usher in the extension phase. During the maintenance phase, GFR reaches a stable nadir as cellular repair processes are initiated in order to maintain and re-establish organ integrity. The recovery phases in marked by a return of normal cell and organ function than results in an improvement in GFR. Adapted from (532). 

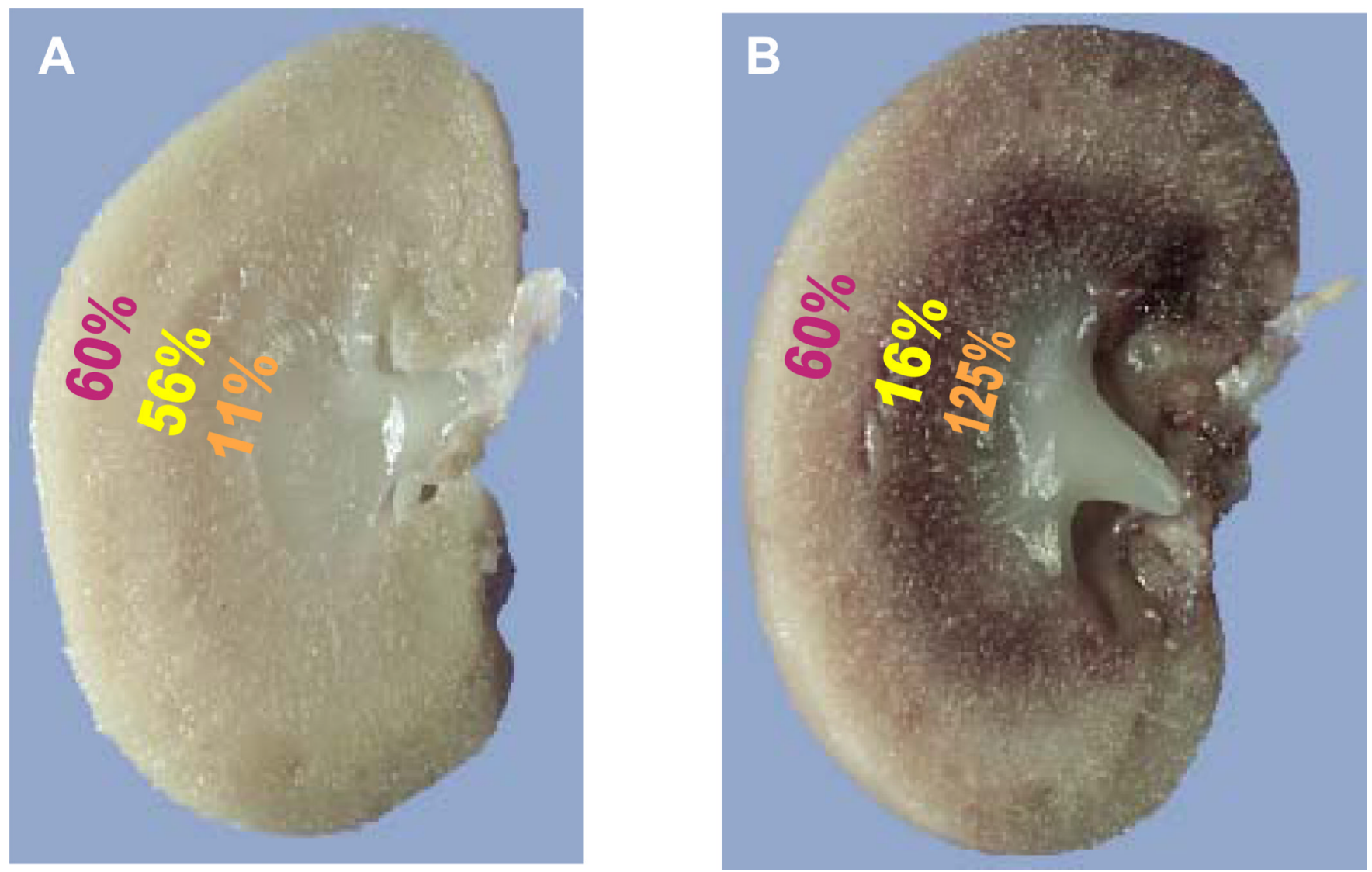

Figure 2. Regional blood flow is altered following injury in ischemic AKI

Immediately following ischemic injury total renal blood flow is reduced but more striking are the regional deficits in blood flow that exist in the cortex, outer stripe of outer medulla and inner stripe of the outer medulla as indicated in panel A (data from (251)). As overall blood flow starts to recover in the ensuing hours after injury, profound regional alterations in blood flow remain with progressive and profound reduction of the blood flow to the outer stripe of the outer medulla as indicated in B (data from (202)). 


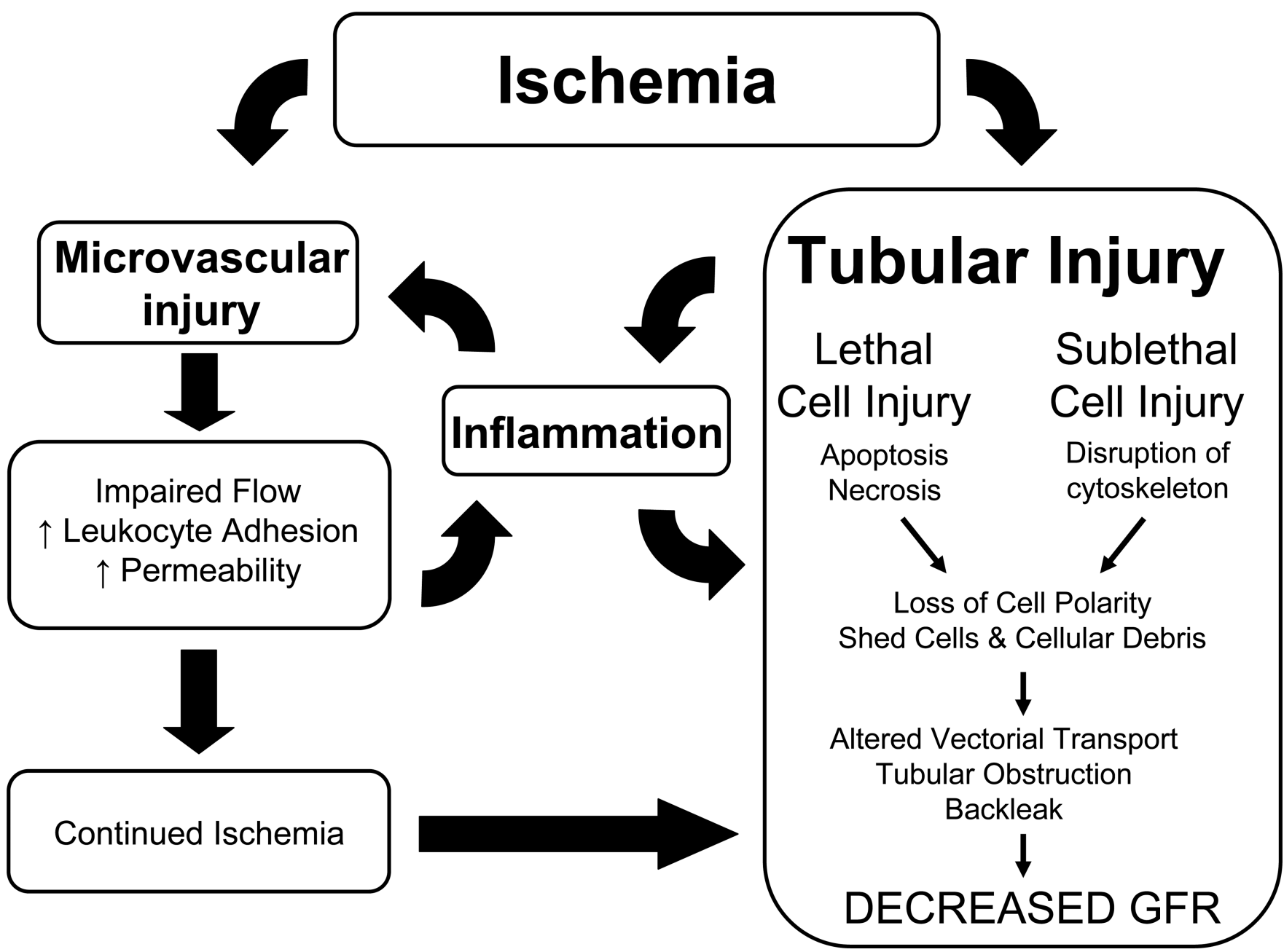

Figure 3. Interplay between tubular and vascular injury leading to sustained reductions of GFR in the extension phase of AKI

Injury induced by ischemia can results in damage to both the tubular as well as the microvascular compartment. Resolution of vasoconstriction appears effective at reducing injury when administered prophylactically, but not following established injury. Resistance may be due to exacerbated inflammation, which may impart reductions in RBF and GFR insensitive to vasodilator therapies. Of central importance in this process is the activation of inflammatory processes which are influenced by factors released by damaged proximal tubules as well as adhesion of damaged microvascular cells. Infiltrating leukocytes may impinge on RBF either by secreting vasoactive factors, or by contributing to the disruption of flow by physical interference. In addition, exacerbated hypoxia leading to tubular obstruction may contribute to reductions in GFR independent of vasodilator therapy. From citation (531) 

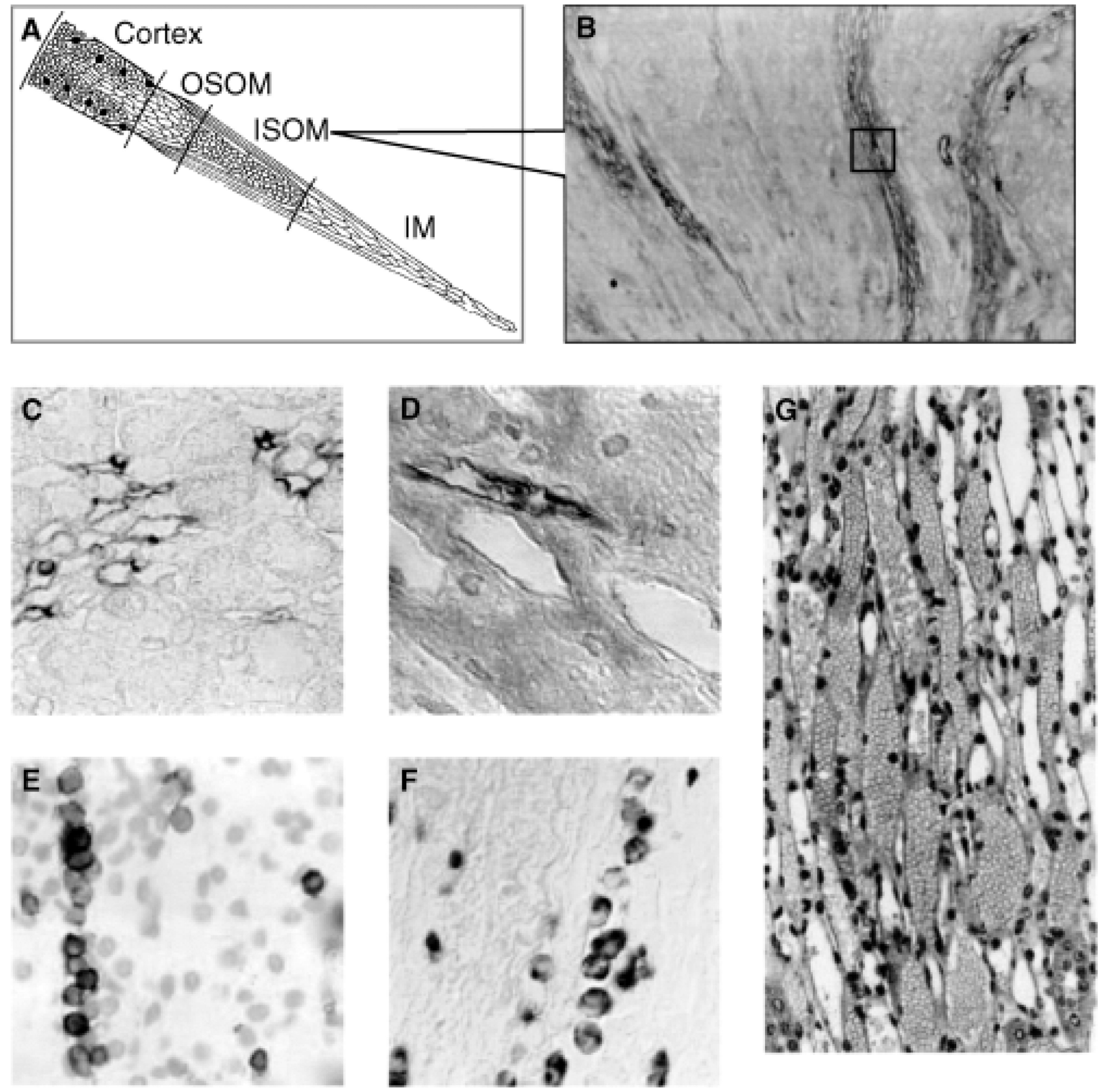

Figure 4. Organization of vascular compartment in the kidney

(A) The medulla is arterial supplied from the efferent arterioles of the juxtamedullary glomeruli, giving supply to the descending arterial vasa recta, and further to the ascending venous vasa recta, draining into the arcuate veins. Abbreviations are: OSOM, outer stripe of outer medulla; ISOM, inner stripe of outer medulla; IM, inner medulla. (B) Very strong expression of b130-1, 2 hours after ischemia/reperfusion (I/R) injury of the kidney, at the level of the ascending vasa recta. (C) Detailed expression of b130-1, 2 hours after I/R injury of the rat kidney, at the level of the ascending venous vasa recta. $(D)$ Detailed expression of b130-1, 2 hours after I/R injury of the human kidney, at the level of the ascending venous vasa recta. $(E)$ Trapping of CD28-expressing T cells in the ascending vasa recta (HIS-17 
staining). $(F)$ Trapping of monocytes/macrophages in the ascending vasa recta (ED-1 staining). ( $G$ ) This trapping of leukocytes in the ascending vasa recta results in an upstream congestion at the ascending arterial vasa recta. This congestion, or no-reflow, represents a well known phenomenon in acute ischemic injury, exacerbating during reperfusion the ischemic damage. From citation (626). 


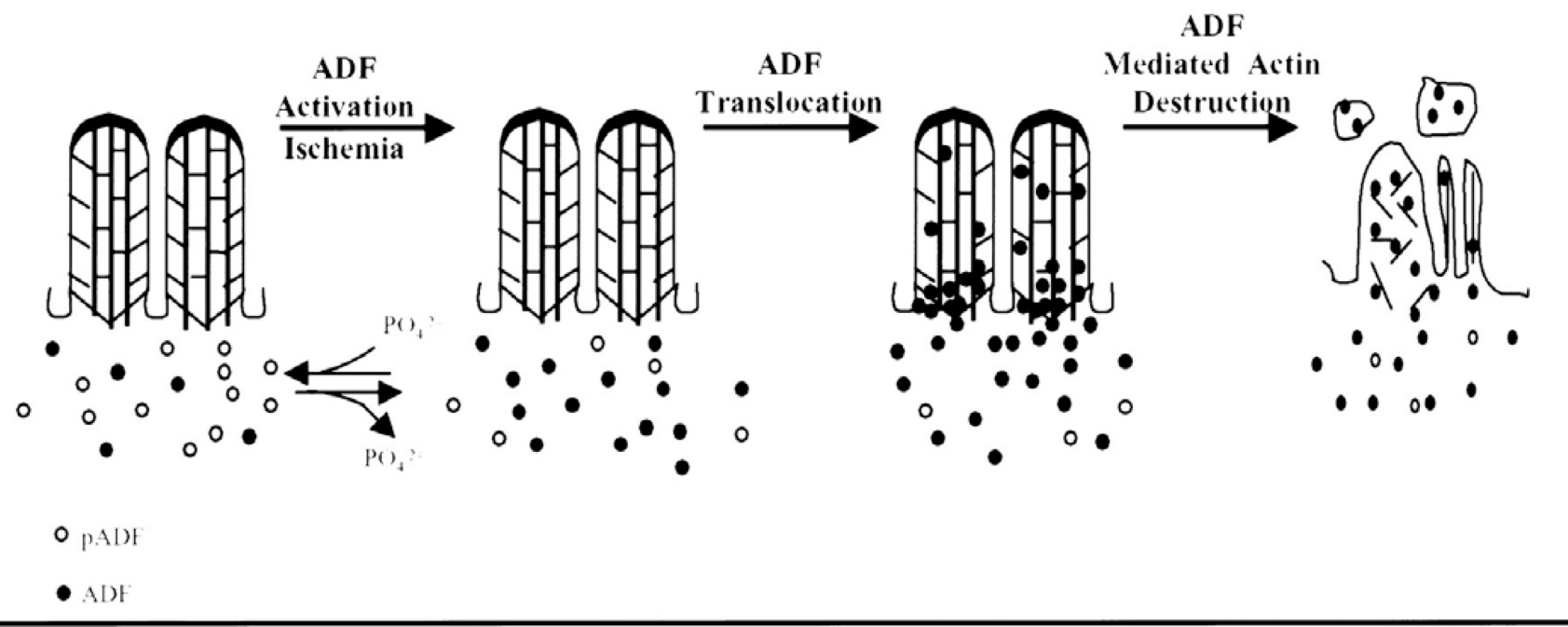

Figure 5. A proposed model for the dephosphorylation, activation, and translocation of ADF to the apical microvilli during ischemia

Under physiological conditions, the distribution of ADF and phosphorylated ADF (pADF) in proximal tubule cells is diffuse throughout the cytoplasm with little or no localization to the apical microvillar region. With ischemia, pADF is dephosphorylated and, therefore, activated. In addition, the diffuse cytoplasmic localization of ADF changes with ADF now concentrating at the apical membrane region of the cell. It is hypothesized that ADF relocalizes to the apical microvillar region and binds the microfilament core, resulting in markedly enhanced filament severing and depolymerization. Breakdown of the microfilament core is accompanied by dramatic changes in the overlying microvillar membrane. The microvillar membrane is internalized or extruded as membrane vesicle or blebs. These vesicles contain both ADF and monomeric actin. Figure and legend from citation (15) 

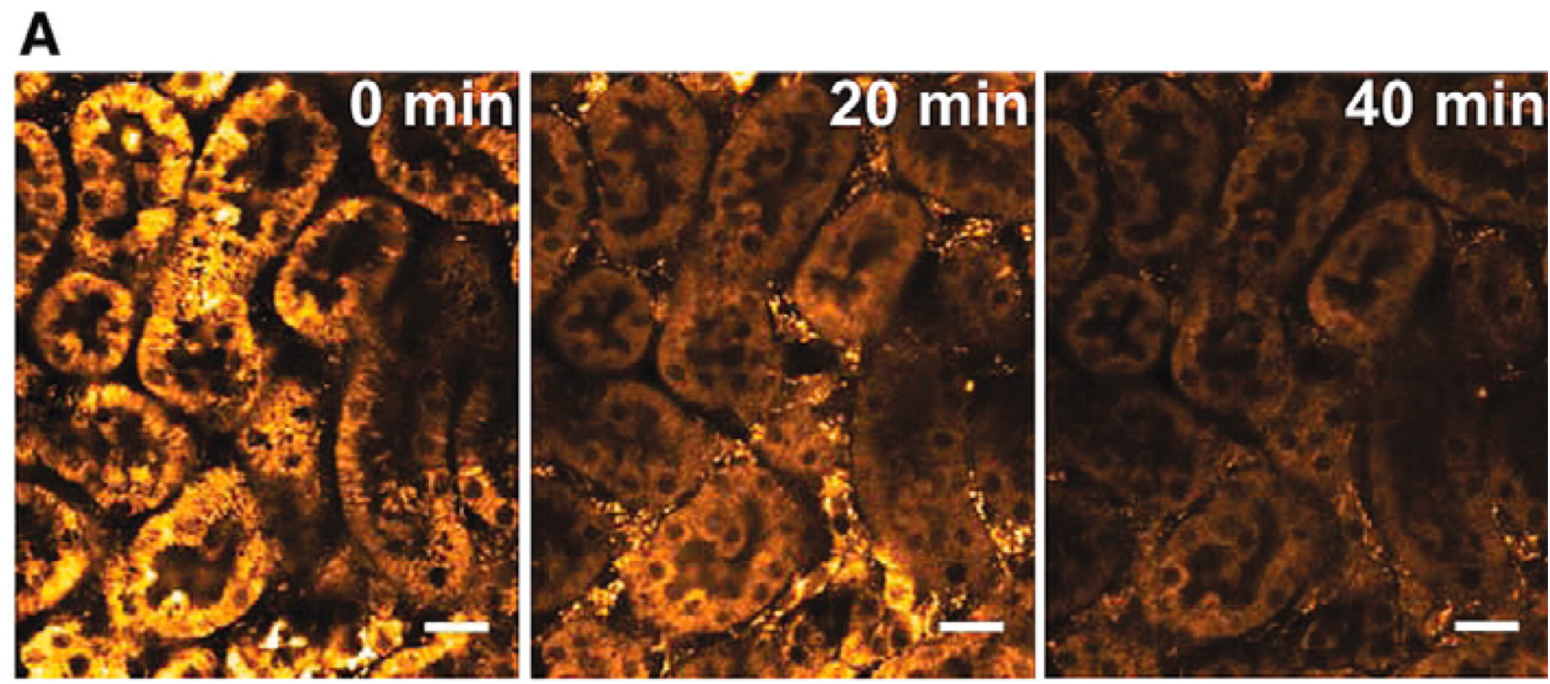

B

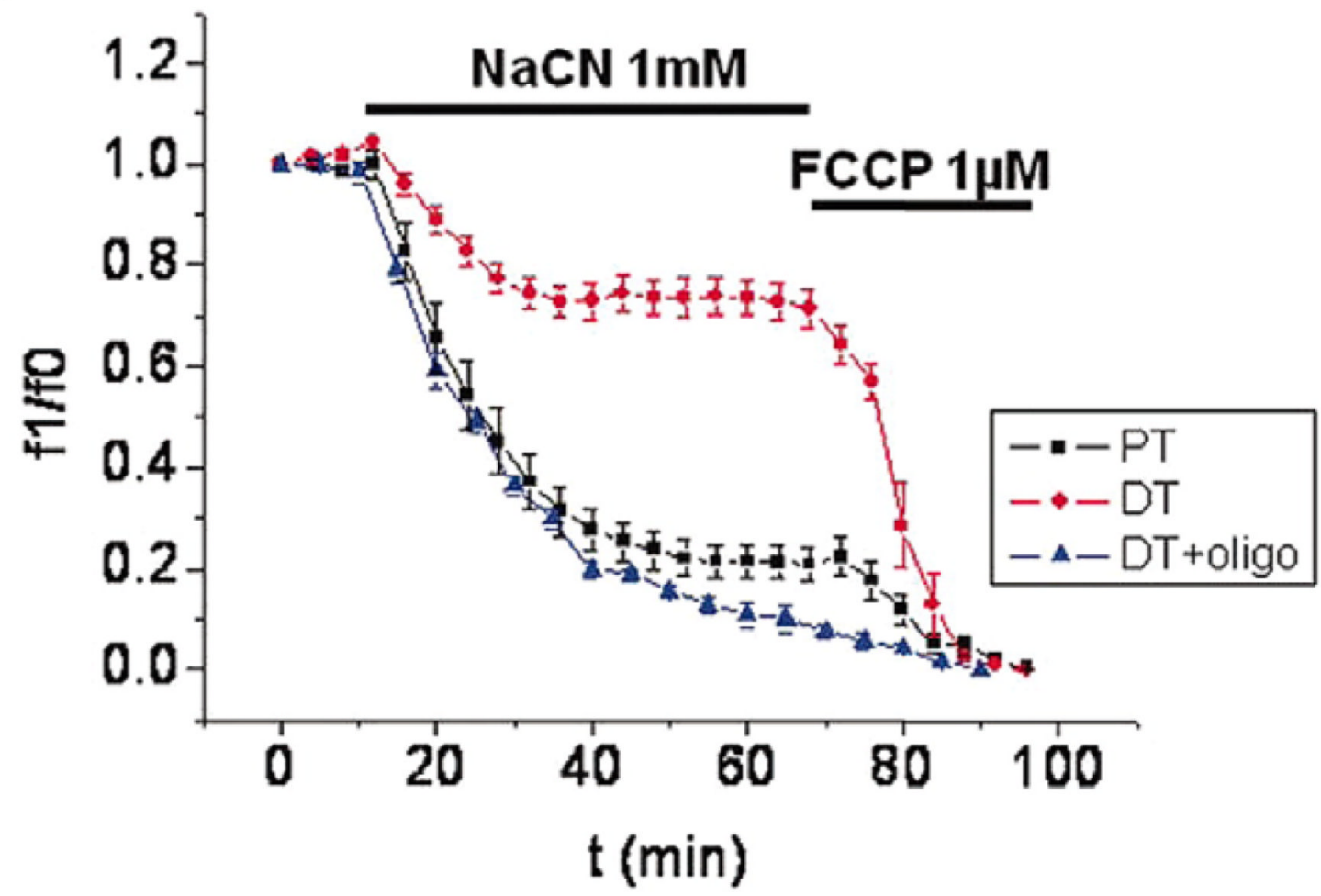

Figure 6. Evidence of mitochondrial depolarization in kidney tissue slices by chemical anoxia using multiphoton imaging

PTs loaded with TMRM showed rapid depolarization of $\Delta \psi \mathrm{m}$ after chemical anoxia. Bar $=$ $20 \mu \mathrm{m}$. (B) In the DT, the decrease was slower and $\Delta \psi \mathrm{m}$ was not completely depolarized after $60 \mathrm{~min}$ of anoxia; however, in the presence of oligomycin $(5 \mu \mathrm{g} / \mathrm{ml}), \Delta \psi \mathrm{m}$ depolarized rapidly in distal tubular cells when exposed to anoxia. Data are means \pm SE signal per tubule from a total of 15 PTs, 15 DTs without oligomycin, and 29 DTs with oligomycin from three separate slices for each experiment. The data were normalized from 1 (value at $\mathrm{t}=0$, taken as resting $\Delta \psi \mathrm{m}$ ) to 0 (minimum value after FCCP, taken as $0 \mathrm{mV}$ ). Data from citation (185). 
A

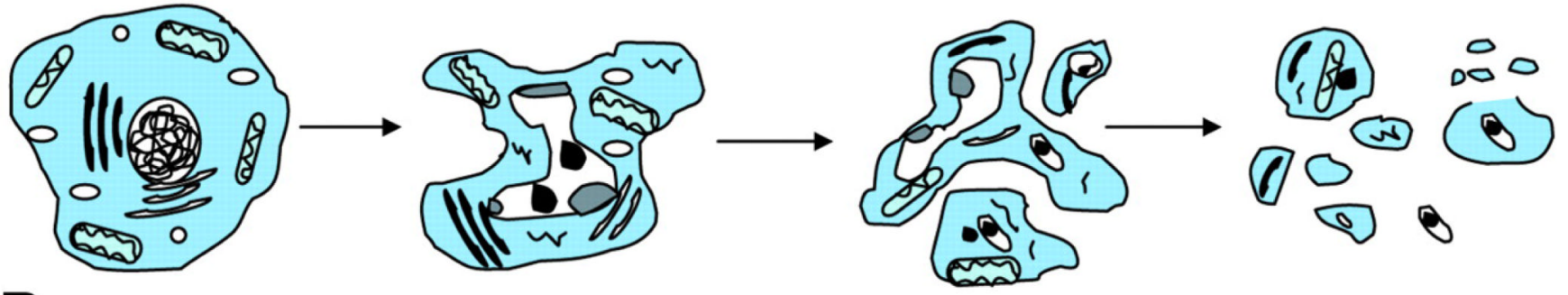

B

Normal cell

Condensation
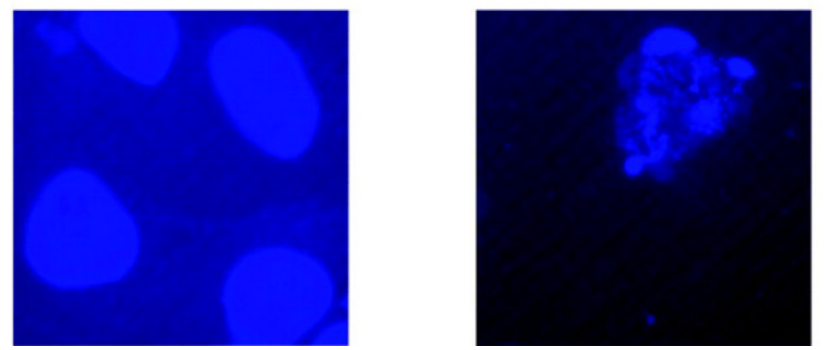

Fragmentation

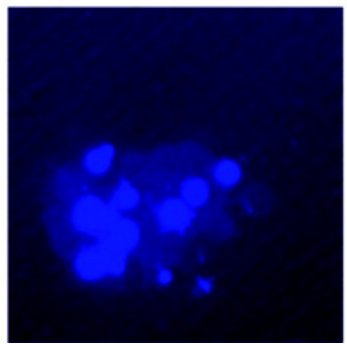

Apoptotic bodies and
Secondary necrosis

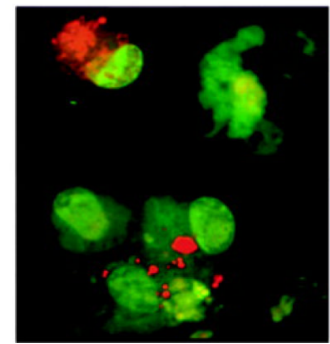

Figure 7. Illustration of the various stages of apoptotic cell death

A: depiction of the stereotypical changes including condensation, changes in nuclear structure, and fragmentation of the cell into small apoptotic bodies. In vivo, the apoptotic bodies are phagocytosed by neighboring cells, whereas in vitro they undergo swelling and eventual lysis (secondary necrosis). B: photographs of LLC-PK1 cells undergoing apoptosis at the corresponding stages as shown in A. Apoptosis was induced by overnight exposure of the cells to $50 \mu \mathrm{M}$ cisplatin. The cells in the first 3 photographs were stained with Hoechst dye, and the cells in the last photograph were stained with acrydine orange and ethidium bromide. In the last photograph, viable cells appear green, whereas the apoptotic cells with intact plasma membrane appear green with yellowish dots representing condensed chromatin; apoptotic cells and bodies that are undergoing secondary necrosis appear bright orange or red due to the plasma membrane damage and entry of ethidium bromide.

Illustration from citation (415). 


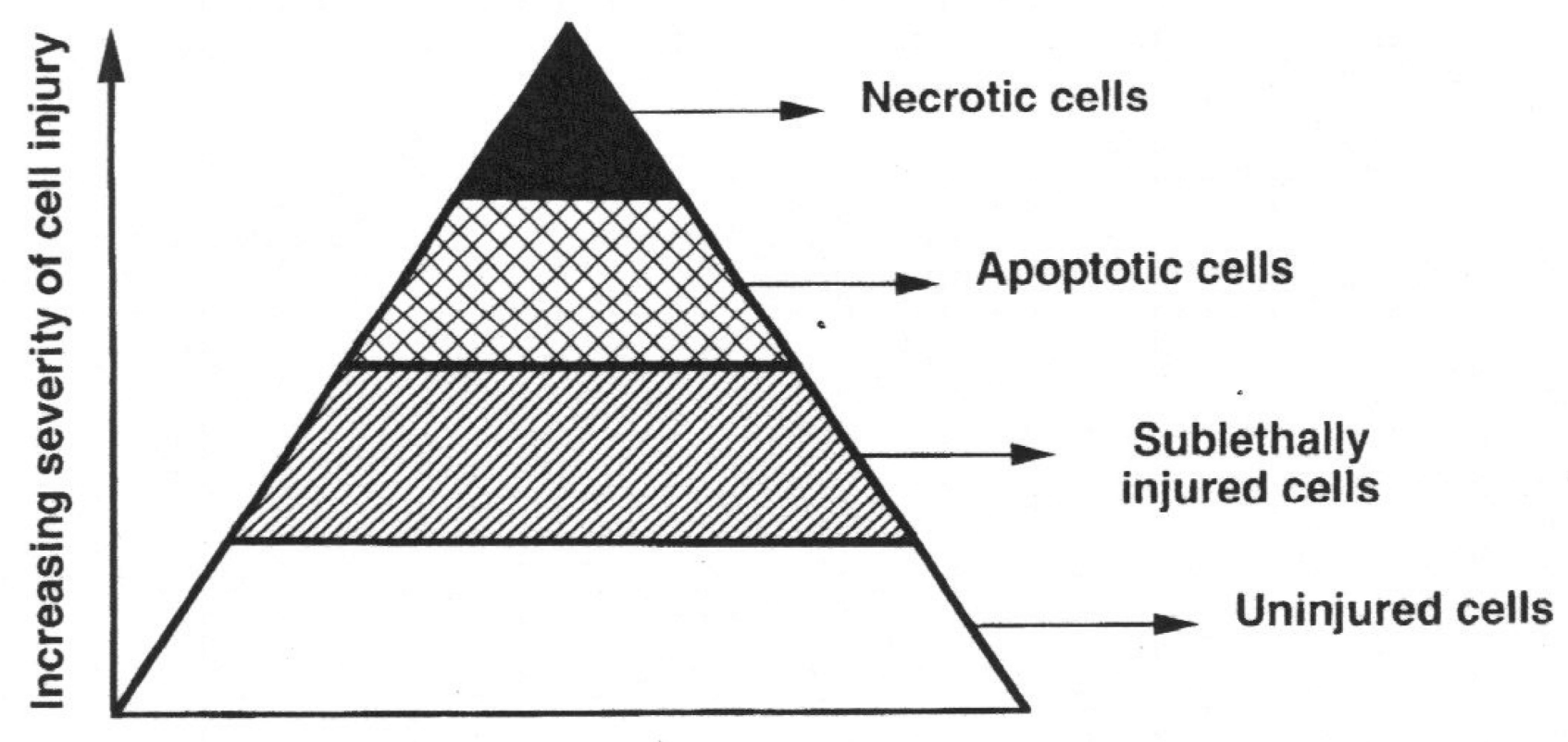

Figure 8. The continuum of renal cell damage

Individual renal tubular cells are likely to respond in different ways to injury depending upon the severity of the noxious stimulus. The majority of cells presumably remain viable, either because they escape injury altogether, or because they are only sublethally injured and able to recover. More severe injury likely results in apoptosis, whereas necrosis only occurs when cells are subjected to extremely severe injury that leads to critical energy depletion and subsequent metabolic collapse. Legend and figure from citation (320). 


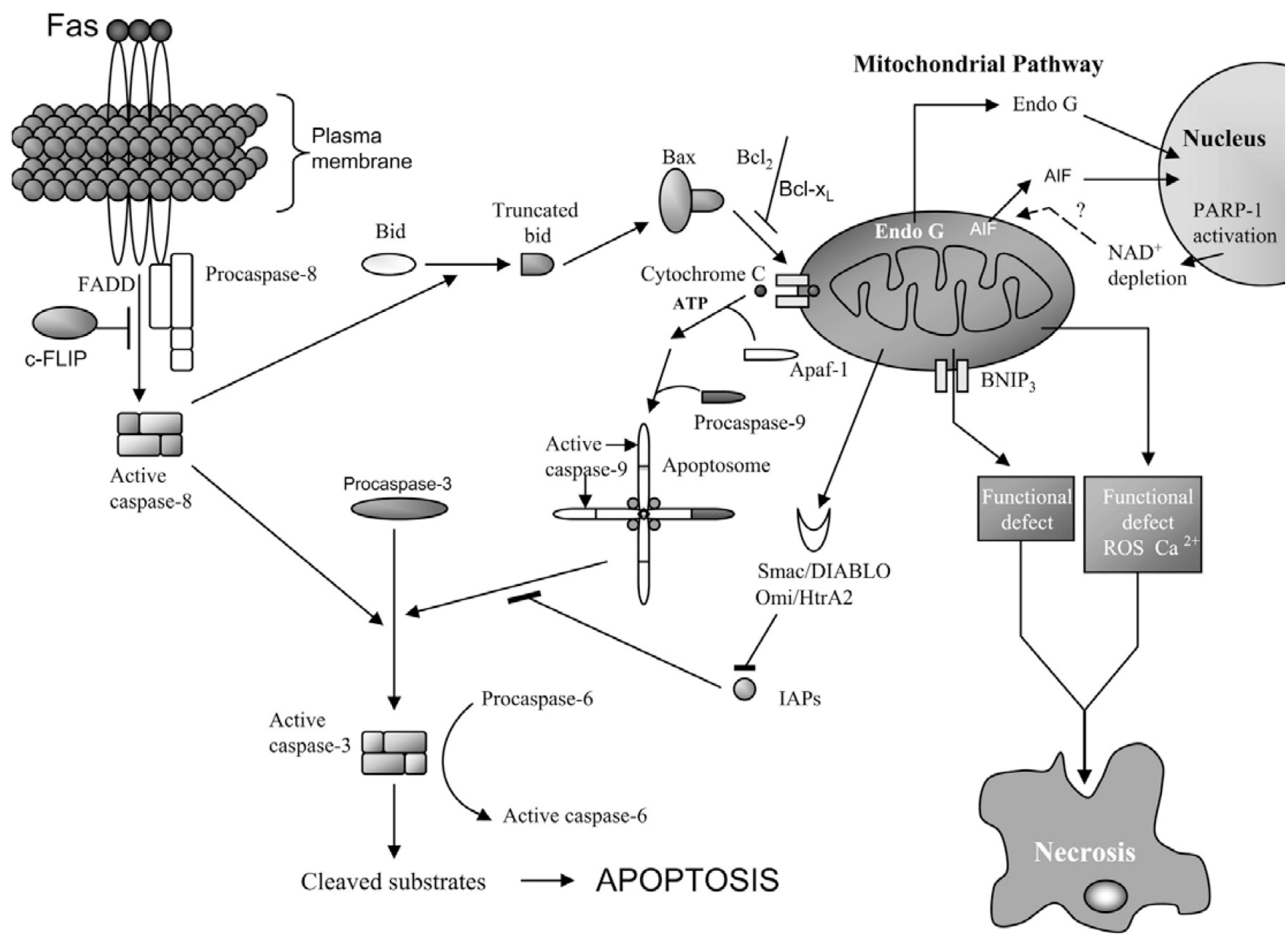

Figure 9. Overview of death-signalling pathways in mammalian cells

The death receptor pathway (left) is initiated by the binding of a ligand (Eg: FasL) to its receptor Fas, which results in the sequential recruitment of FADD and pro-caspase-8. cFLIP can block the recruitment of pro-caspase- 8 to the complex. The proximity of several pro-caspase- 8 molecules results in its activation. Caspase- 8 can proteolytically activate caspase-3, or it can cleave Bid to its truncated form t-Bid, which binds to Bax and gets integrated into the mitochondrial membrane to release cytochrome $\mathrm{c}$. In response to various cellular stress-induced apoptotic stimuli, the intrinsic mitochondrial pathway is activated. This pathway involves the translocation of proapoptotic molecules such as Bax from the cytosol to the mitochondrial membrane. Bax can release cytochromec from the mitochondria into the cytosol. Cytochromec associates with Apaf-1 and caspase-9 to form the apoptosome and subsequent activation of caspase-3. Mitochondria also release AIF and Endo G, which may exert their effects on the nuclei. Mitochondria released Smac/Diablo and Omi/HtrA2 sequesters inhibitors of apoptosis (IAPs) to prevent them from inhibiting caspase-3. BNIP3 is a $\mathrm{Bcl} 2$ family member that is translocated and integrated into the mitochondria. Unlike other Bcl2 family members, BNIP3 can induce necrotic cell death in response to death stimuli. Activation of poly (ADP-ribose) polymerase (PARP) leads to NAD+ depletion and may induce mitochondrial depolarization to release AIF. ROS, reactive oxygen species. Legend and Figure from citation (320). 


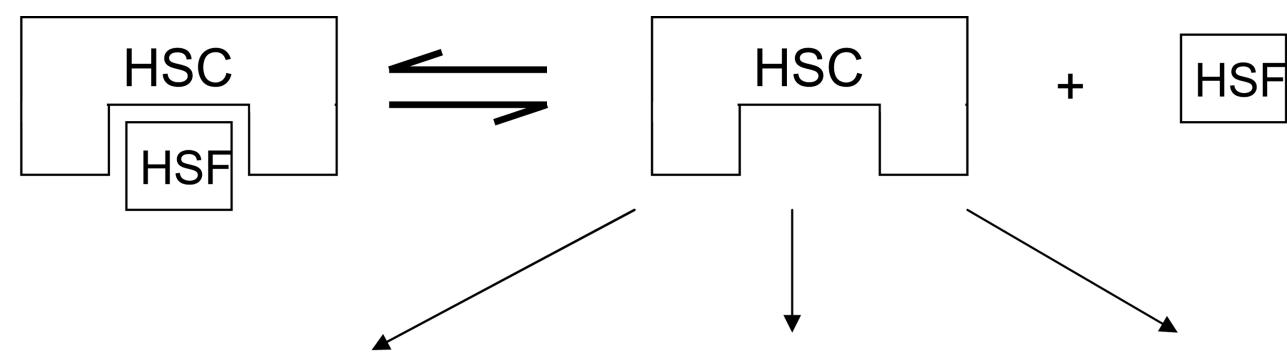

New Protein

Synthesis

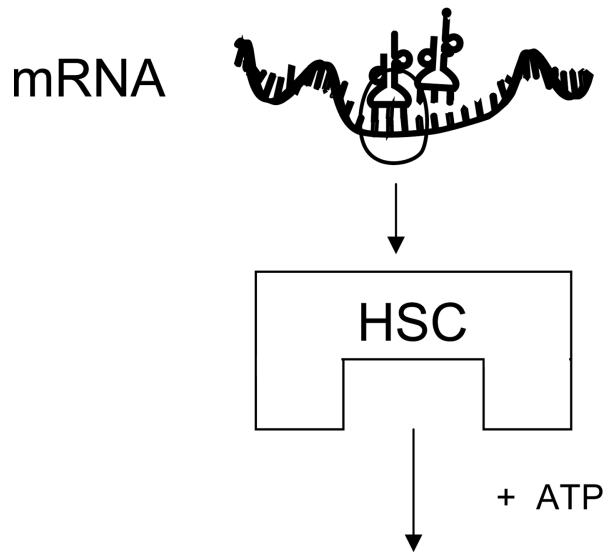

Folding

Protection Assembly
Native

Protein
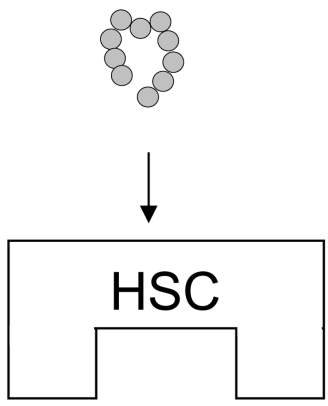

Sorting

Targeting Refolding
Denatured Protein
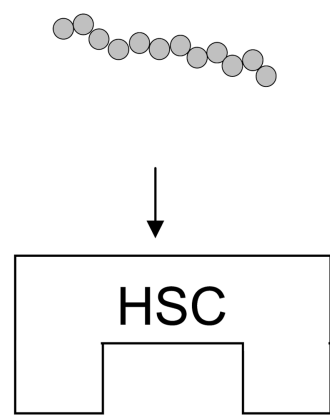

+ ATP

Stabilize

Repair Disposal

Figure 10. Control of HSP expression in response to cell stress

Shown are the known actions of the constitutively expressed Hsps, primarily of the Hsp70 family called heat shock congnates (HSC) in processing cellular functions. Cell stress increases denatured proteins increasing the demand for HSC. Heat shock transcription factor (HSF), reversibly binds to HSC and is released with the increased demand for HSC. HSF then rapidly initiates transcription for all inducible Hsps including Hsp 70 and Hsp 25/27. Figure from citation (521). 

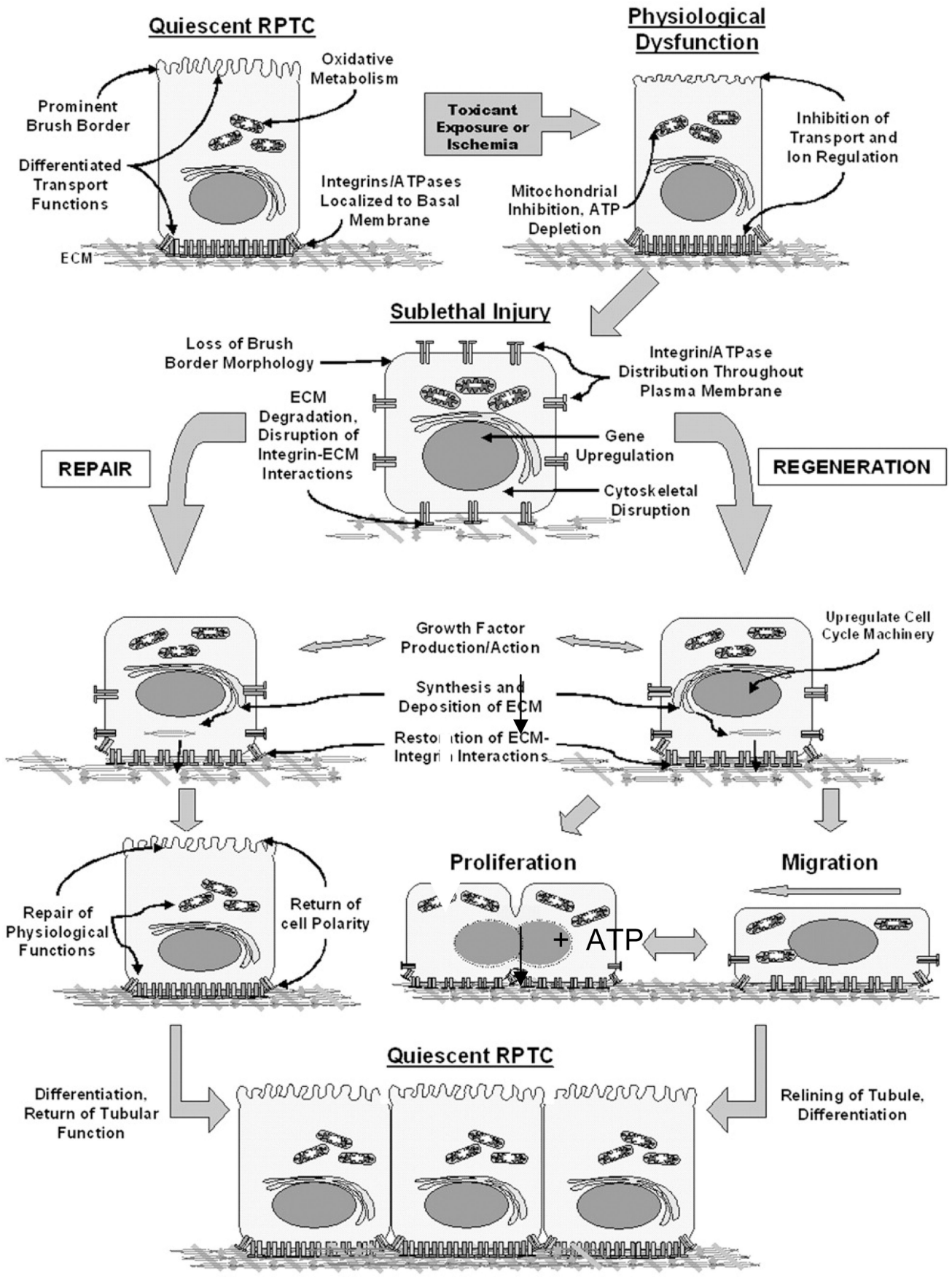

Figure 11. Repair and regeneration of renal proximal tubule cells following acute sublethal injury

Sublethally injured RPTC either repair physiological functions and restore normal tubular function or dedifferentiate, migrate, and/or proliferate to replace lost cells, then differentiate and resume normal function. The processes of repair and regeneration work in concert to ensure relining of the damaged nephron and restoration of renal function. From citation (402). 

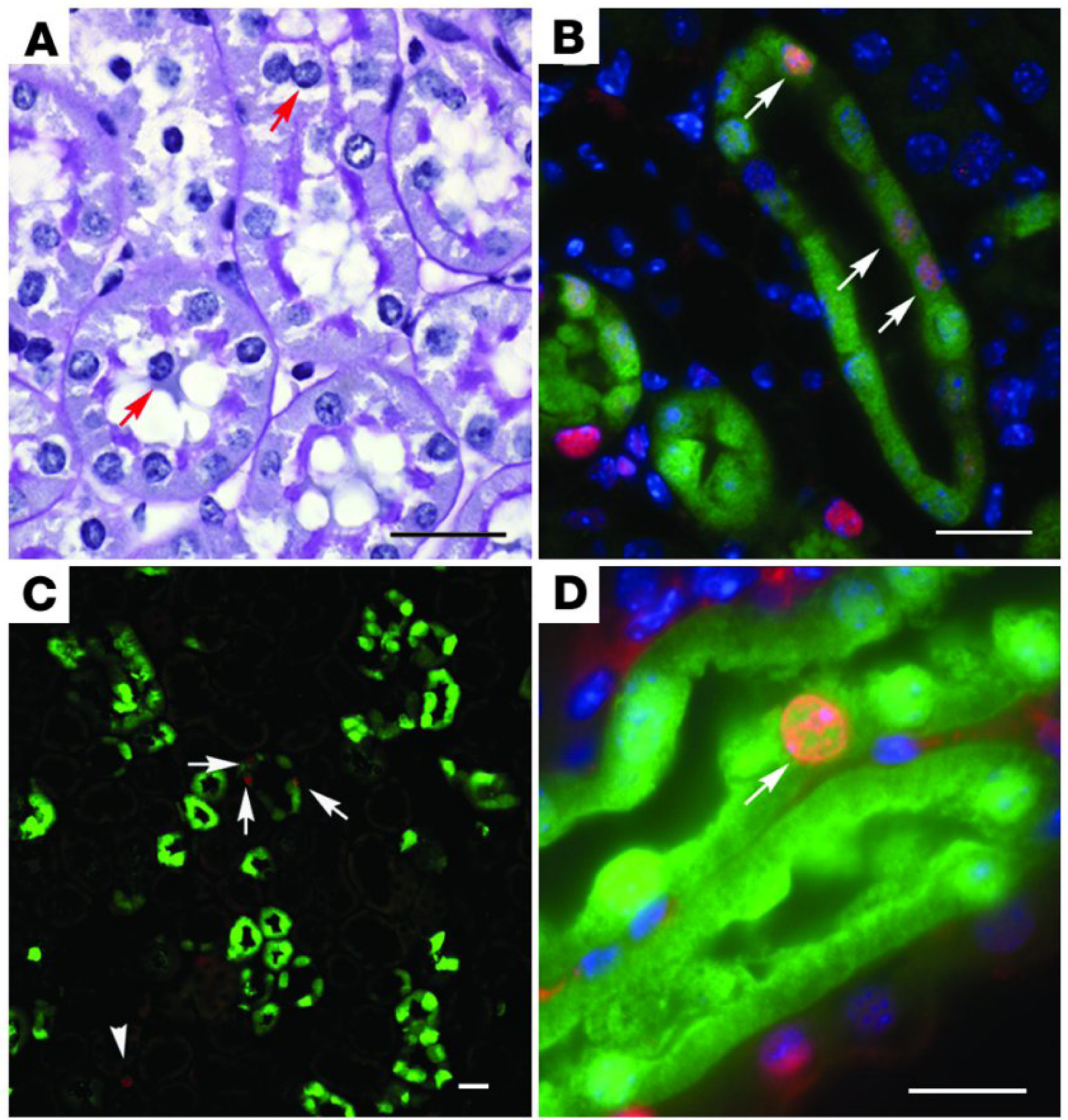

Figure 12. Evidence that sublethally damaged proximal tubules are the source of dividing cells during recovery from AKI

PAS staining and immunostaining were performed in the kidney sections of cre ${ }^{\mathrm{ksp}} ; \mathrm{Z} / \mathrm{EG}$ mice with renal IRI. (A) PAS staining of the kidney at 2 days after IRI. Tubular injury is shown by the loss of brush border membrane, cell detachment from the basement membrane, and nuclear condensation in some cells (arrows). (B) Expression of PCNA in tubular cells (red, arrows). (C) Low-power image of BrdU incorporation in renal tubules. Some BrdU-containing cells (red, arrows) colocalized with EGFP-expressing cells (green). The arrowhead indicates BrdU incorporation in an EGFP-negative cell. (D) BrdU incorporation (red, arrow) in the epithelial cells expressing EGFP (green). Note: the cre 
transgene labels tubular cells and their progeny with EGFP. The nuclei were counterstained with DAPI, and images were merged (B-D). Scale bars: $20 \mu \mathrm{m}$. From citation (325). 

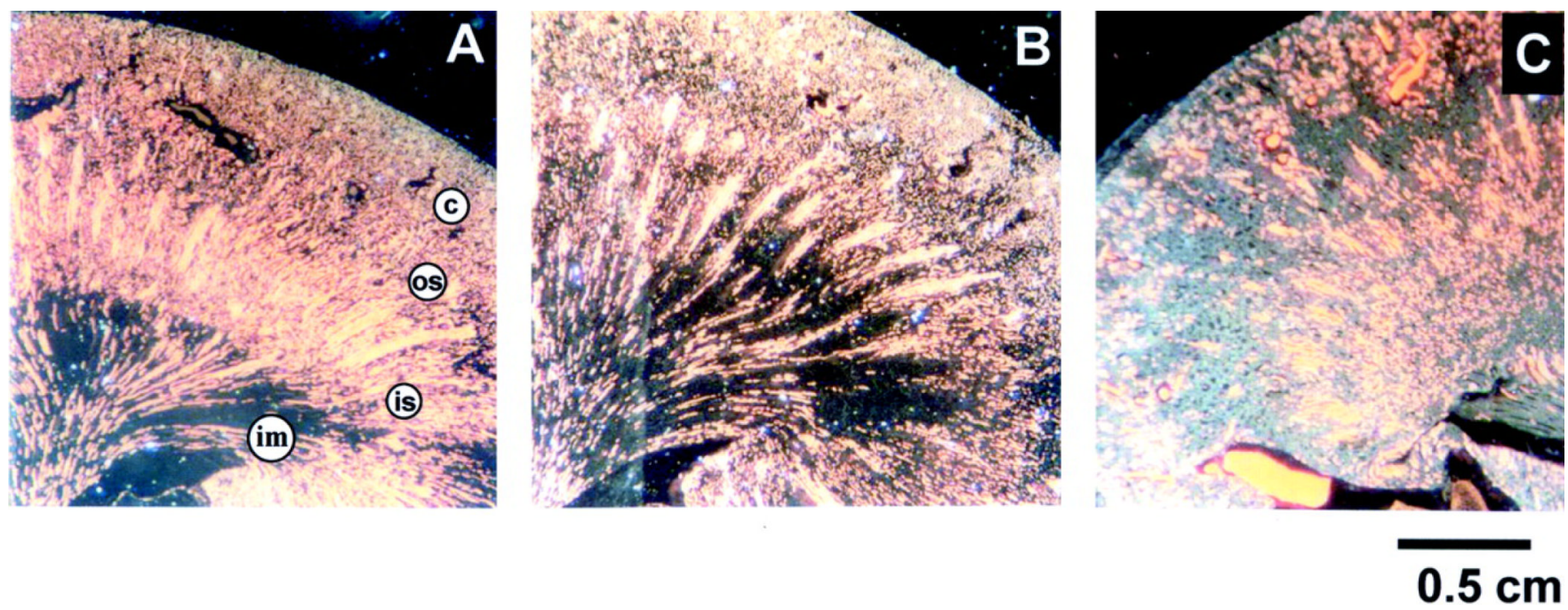

Figure 13. Gross renal morphology and capillary filling in normal and postischemic kidneys Representative stereoscopic views of 20- $\mu \mathrm{m}$ Microfil-infused kidney section. Shown are Microfil-infused kidneys from a sham-operated rat (A) at 4 (B) and $8 \mathrm{wk}(\mathrm{C})$ post-ischemic injury. In this stereoscopic view, Microfil appears as bright yellow against a dark background. A reduction in Microfil-infused structures in recovered postischemic kidneys is evident. c, cortex; os, outer stripe of the outer medulla; is, inner stripe of the outer medulla; im, inner medulla. Magnification is shown. From citation (32). 


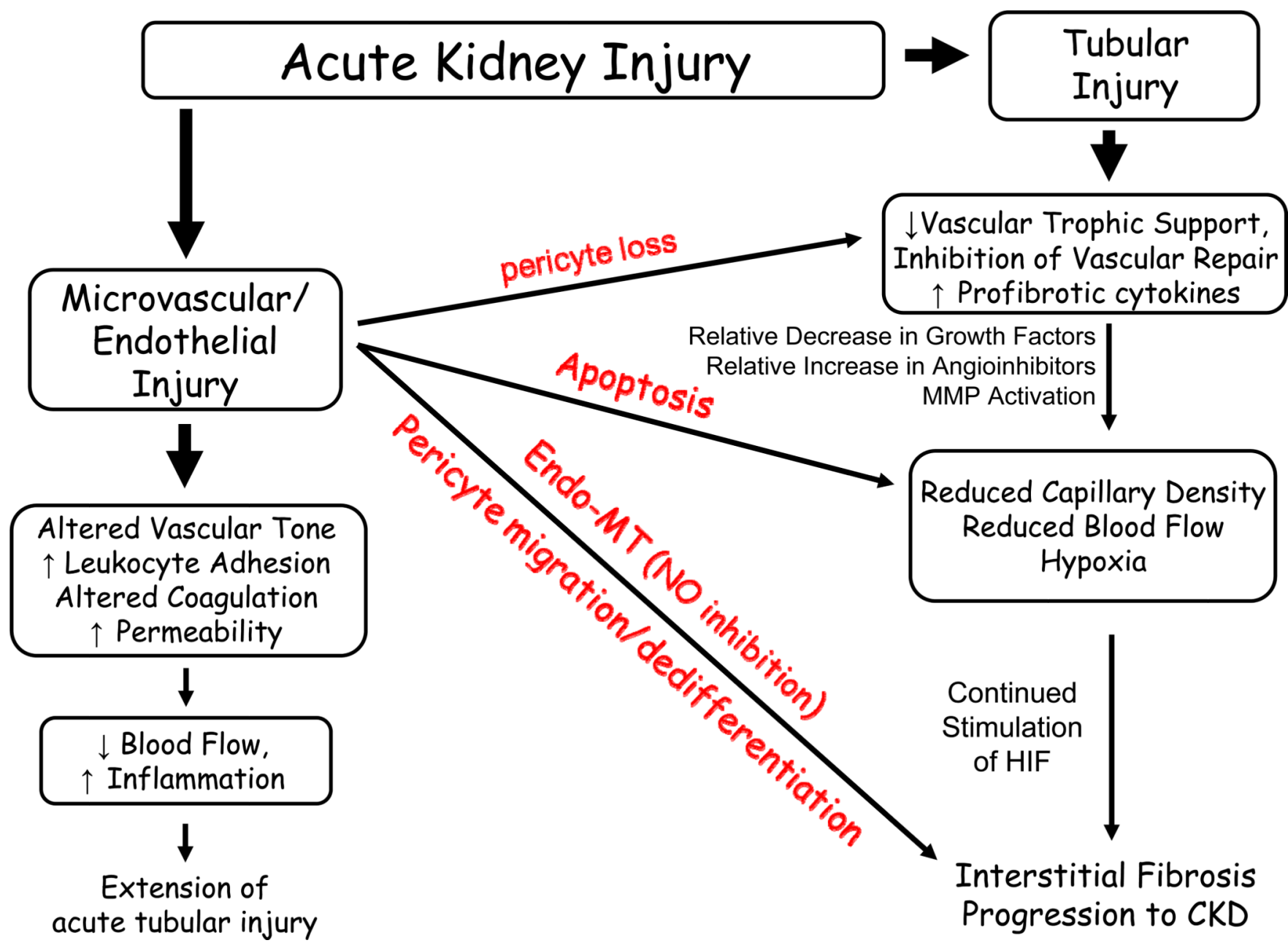

Figure 14. A potential role for vascular dropout in promoting the development of CKD following AKI

Acute injury has the potential to affect both tubular and vascular compartments. In addition to direct injury to the microvascular compartment, tubular injury may compromise normal vascular support, shifting the environment to one which promotes vascular impairment rather than vascular stability, including the loss of VEGF expression, the increase in TGFbeta expression as well as several other angio inhibitory compounds. The resultant decrease in capillary structures has a number of potential consquences on renal function including the exacerbation of hypoxia and the impairment of $\mathrm{Na}$ handling hemodynamic responses. Hypoxia, along with the potential endothelial mesenchymal transition are likely to participate in the development of fibrosis, which is also influenced by sustained immune/ inflammatory activity. The figure modified from and earlier version published in citation (29). 